EXTERNAL-ROTOR 6/10 SWITCHED RELUCTANCE MOTOR FOR AN ELECTRIC BICYCLE 


\title{
EXTERNAL-ROTOR 6/10 SWITCHED RELUCTANCE MOTOR FOR AN ELECTRIC BICYCLE
}

\author{
By \\ JIANING LIN, B. Eng.

\begin{abstract}
A Thesis
Submitted to the School of Graduate Studies

In Partial Fulfillment of the Requirements

For the Degree of

Masters of Applied Science
\end{abstract} \\ McMaster University \\ CCopyright by Jianing Lin, December 2013
}


Master of Applied Science (2013)

(Mechanical Engineering)

TITLE:

AUTHOR:

SUPERVISORS:

NUMBER OF PAGES:
External-Rotor 6/10 Switched Reluctance Motor for an Electric Bicycle

Jianing Lin

Dr. Ali Emadi \& Dr. Nigel Schofield

xvi, 137
McMaster University

Hamilton, Ontario, Canada 
M.A.Sc. Thesis - Jianing Lin; McMaster University - Mechanical Engineering.

\begin{abstract}
As a cost-effective, healthy, and environmentally friendly personal mode of transportation, electric bicycles (E-bikes) are gaining an increasing market share from conventional bicycles and automobiles. Considering the legal rules in Ontario, Canada, a 500W motor makes the E-bike more attractive for travelling use. At the same time, the simple structure, high torque and power density, as well as the low cost of the switched reluctance machine (SRM) makes it a strong candidate for E-bikes.
\end{abstract}

In this thesis, a 3-phase, external-rotor SRM with 6 stator poles and 10 rotor poles is designed for E-bike. The design of an external rotor arrangement of the 6-10 SRM topology has not previously been reported, hence it offers a new contribution to the published works. The machine design is initiated by the output power equation and is followed by a comprehensive finite element analysis (FEA). The external-rotor arrangement is chosen to facilitate ease of integration into the wheel hub structure of a typical pedal bicycle. The increasing rotor poles yield improved torque ripple reduction than more conventional (i.e. 6-4, 12-8 etc.) SRM design, which is an essential feature for low speed rider comfort.

A new torque ripple reduction control scheme is investigated. Although the comparison shows that the torque sharing function has more positive result than angular position control with regards to torque ripple, this is at the expense of higher losses. Detailed thermal analysis ensures this machine is suitable to require no additional cooling 
M.A.Sc. Thesis - Jianing Lin; McMaster University - Mechanical Engineering.

system. The final machine design is experimentally tested via a full system prototype. Results highlight some limitation of the 2-D FEA in terms of the winding inductance calculation. Here, the end winding introduce more influence on short thickness machine, which will reduce its output power. However, its power-speed curve shows that this prototype machine has very strong overload ability. 
M.A.Sc. Thesis - Jianing Lin; McMaster University - Mechanical Engineering.

\section{ACKNOWLEDGEMENTS}

First, this research was undertaken, in part, thanks to funding from the Canada Excellence Research Chairs Program.

I would also express my special thanks to Dr. Ali Emadi for his help and support throughout my whole master study. His abundant knowledge and proficient skills as well as rigorous academic attitude set a great example for my future career.

My sincere gratitude goes to Dr. Nigel Schofield who put considerable time and effort to help me better understand this project. His constant encouragement and guidance, together with his strong background in electric machine always brightens my mind.

In addition, I would thank all my colleagues in MacAUTO. I would thank Yinye Yang for his experience sharing in prototype machine setup, thank Fei Peng for his help with the converter setup, and thank anyone else who helped me in any different way.

At last but not least, I owe a special debt of gratitude to all my family memeber. It's their love walked me out during those hard times. Without their spiritual and materially support, the completion of this thesis wouldn't be possible. 
M.A.Sc. Thesis - Jianing Lin; McMaster University - Mechanical Engineering.

\section{TABLE OF CONTENTS}

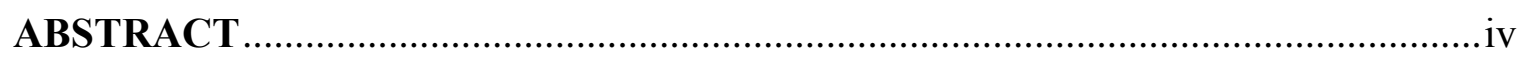

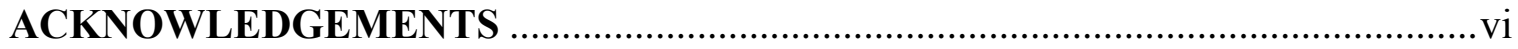

TABLE OF CONTENTS ...................................................................................

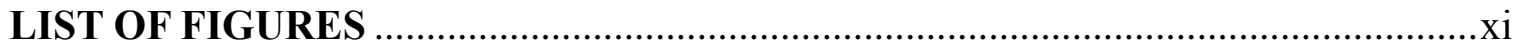

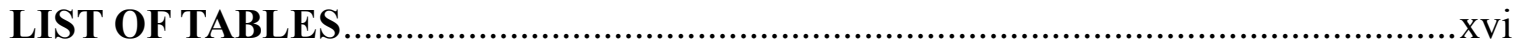

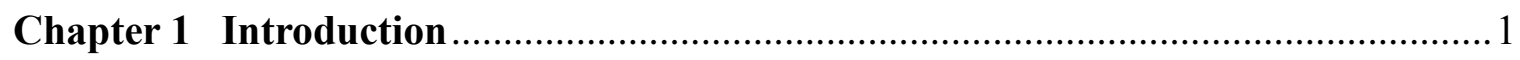

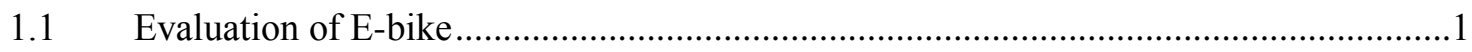

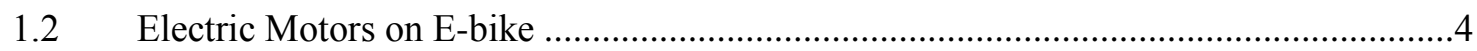

1.3 Switched Reluctance Motor on E-bike .................................................................

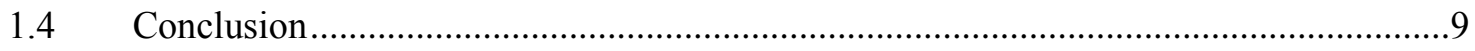

Chapter 2 Fundamental of SRM Design and its Control ................................... 11

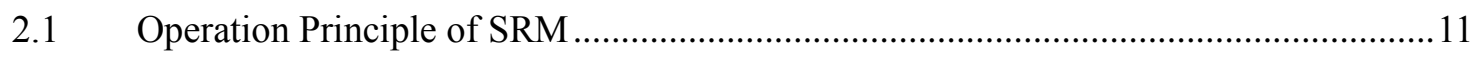

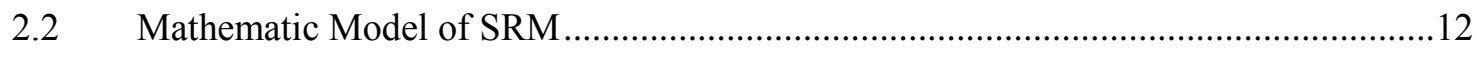

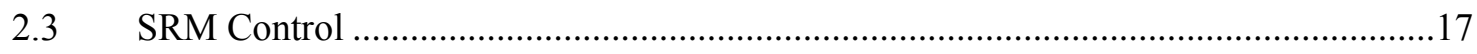

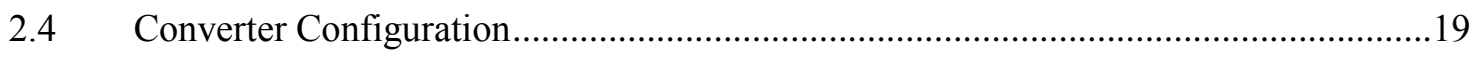

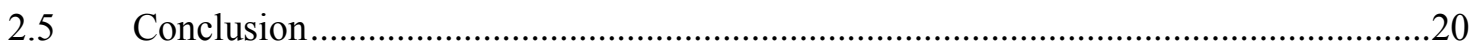

Chapter 3 Design a SRM for Electric Bicycle ................................................ 21

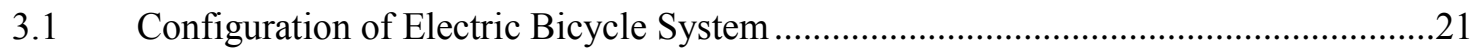

3.1.1 The road load and traction force requirements for the E-bike................................22

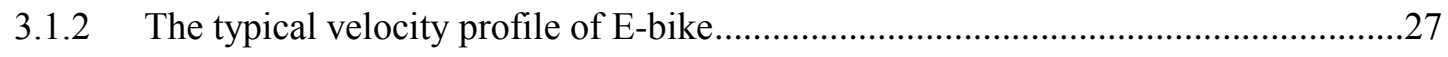


M.A.Sc. Thesis - Jianing Lin; McMaster University - Mechanical Engineering.

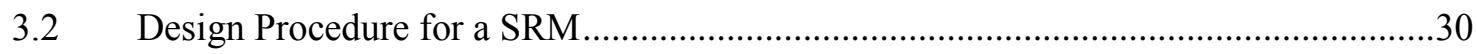

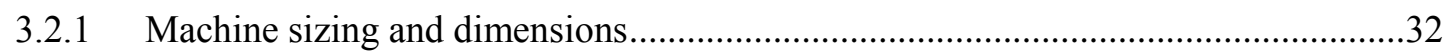

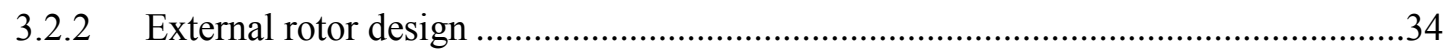

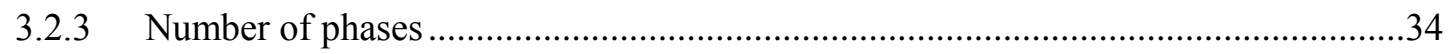

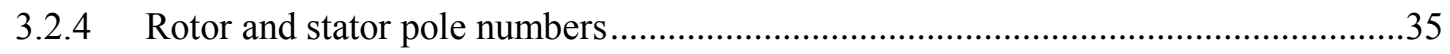

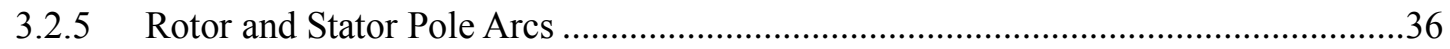

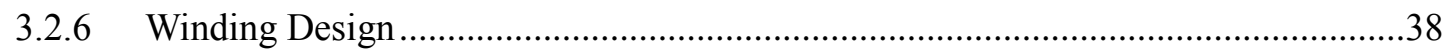

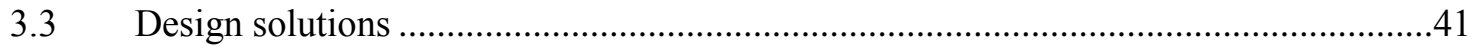

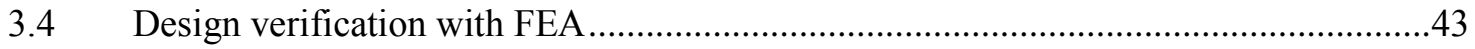

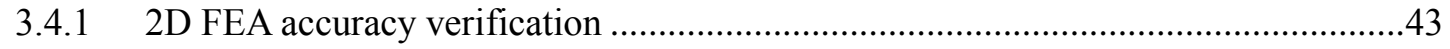

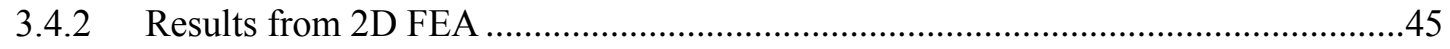

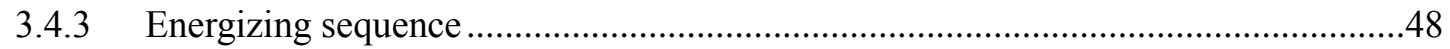

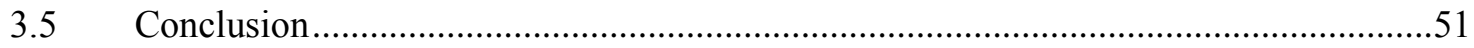

Chapter 4 Switched Reluctance Drive Design and Torque Ripple Reduction .........52

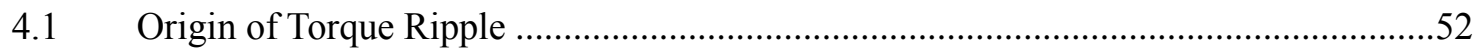

4.2 Classic Control Diagram with Angular Position Control .........................................54

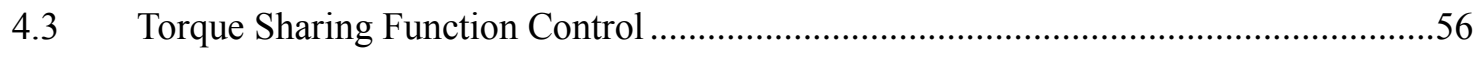

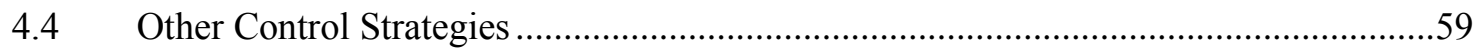

4.4.1 Linearization and decoupling techniques ...........................................................59

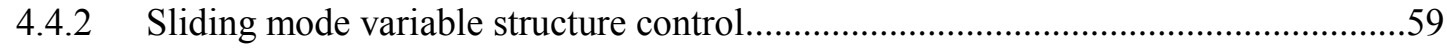

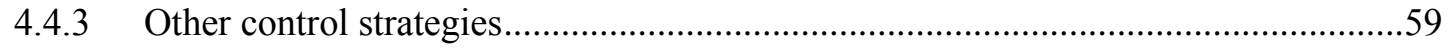

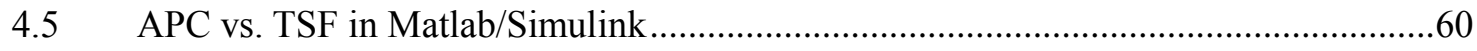

4.5.1 Turn-on/off angle control (angular position control) .............................................60

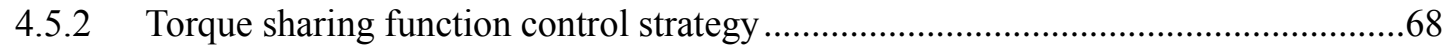


M.A.Sc. Thesis - Jianing Lin; McMaster University - Mechanical Engineering.

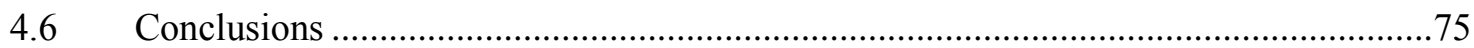

Chapter 5 Thermal Analysis of Designed SRM................................................. 76

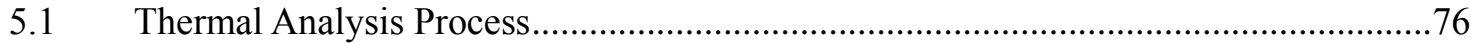

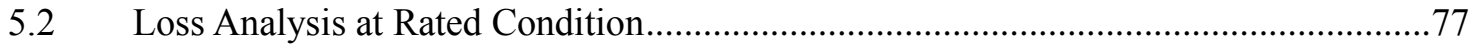

5.3 Average Loss Calculation Considering the Velocity Cycle ........................................79

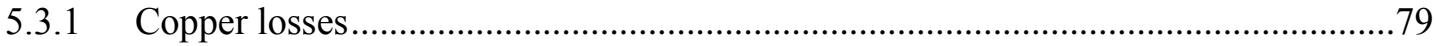

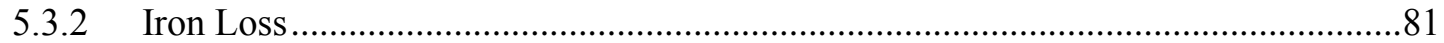

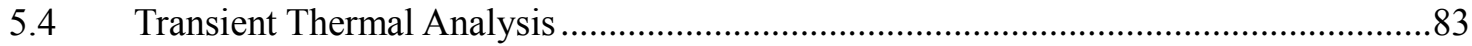

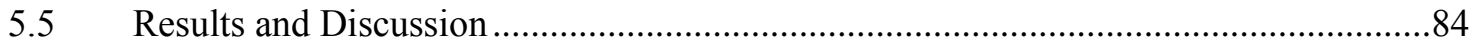

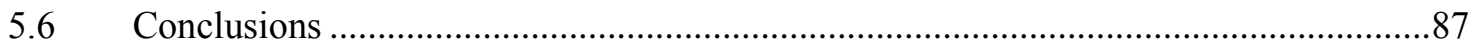

Chapter 6 Prototype Motor Mechanical Realization ............................................ 88

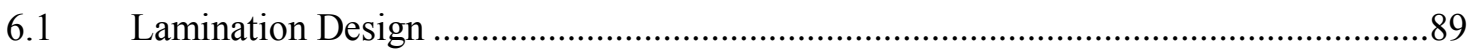

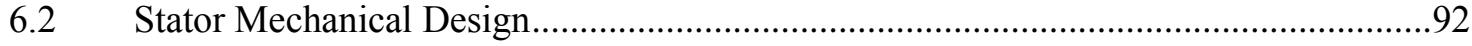

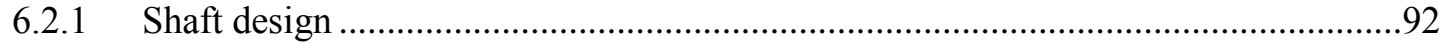

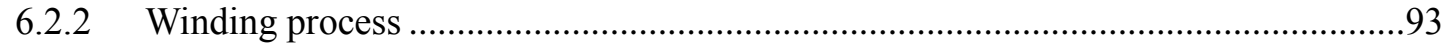

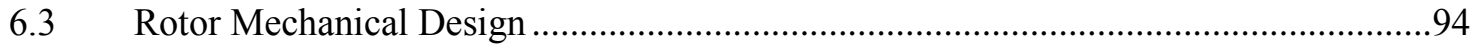

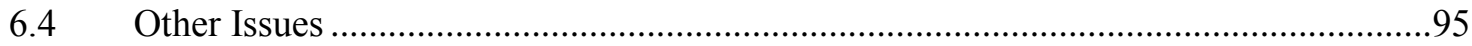

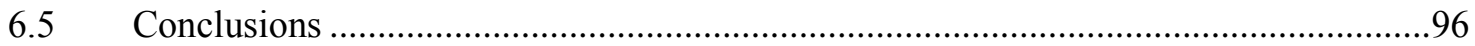

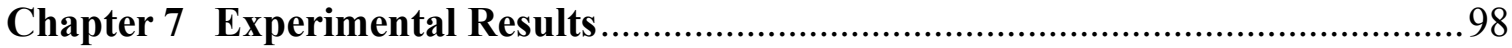

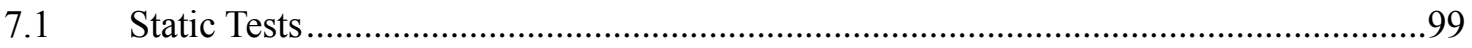

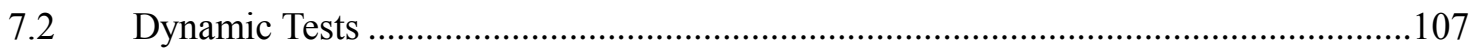

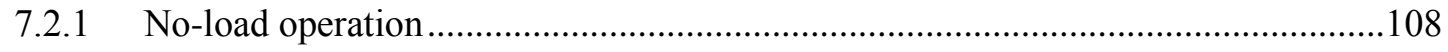

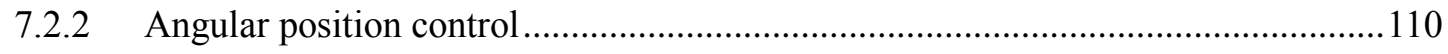


M.A.Sc. Thesis - Jianing Lin; McMaster University - Mechanical Engineering.

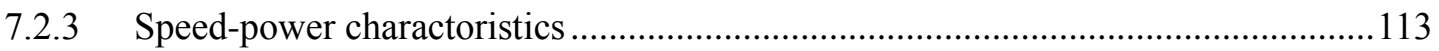

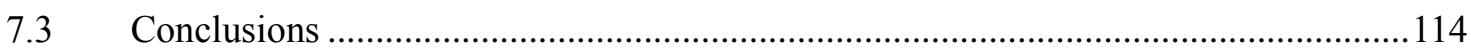

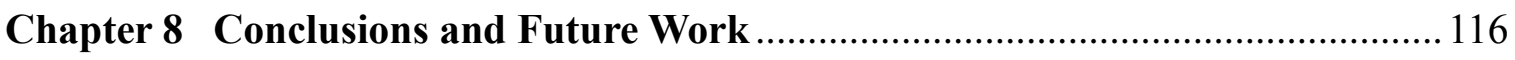

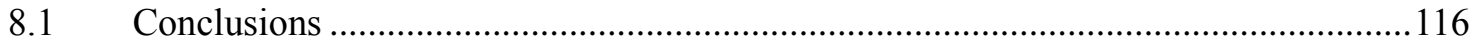

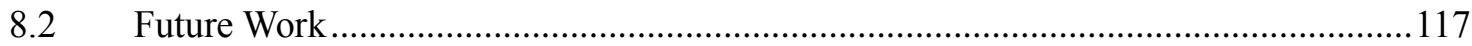

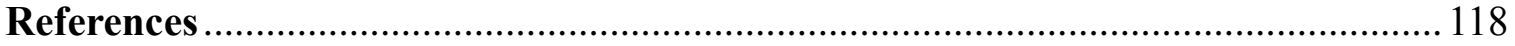

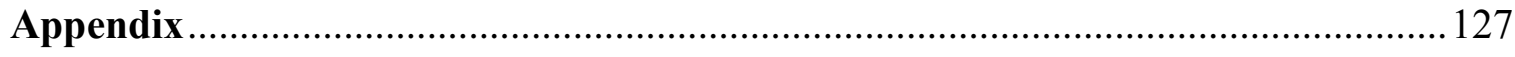


M.A.Sc. Thesis - Jianing Lin; McMaster University - Mechanical Engineering.

\section{LIST OF FIGURES}

Fig. 1.1. A typical parallel hybrid schematic diagram of E-bike........................

Fig. 2.1. SRM operating principle. ..........................................12

Fig. 2.2. Simulation diagram of SRM. ........................................13

Fig. 2.3. Ideal flux-linkage versus phase current profiles for rotor aligned and unaligned commutation positions.. ................................................. 15

Fig. 2.4. Phase inductance characteristics versus rotor position, with motor and generator

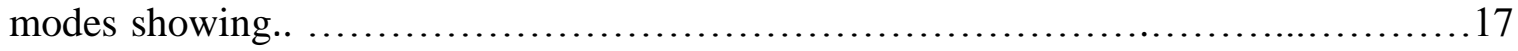

Fig. 2.5. Configuration of asymmetric bridge converter for single phase $\ldots \ldots \ldots \ldots \ldots . . .20$

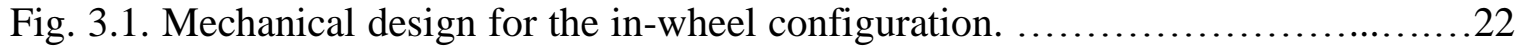

Fig. 3.2. Road load and traction force applied to the E-bike [37]. ....................23

Fig. 3.3. Flow diagram showing human pedal and motor-output torque and power

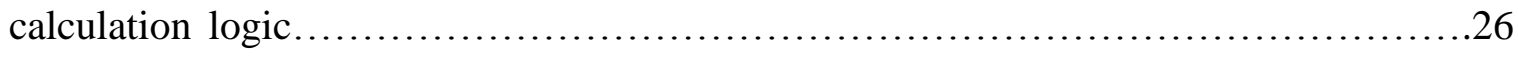

Fig. 3.4. Typical urban bike velocity cycle...................................27

Fig. 3.5. Typical urban bike acceleration cycle. ................................28

Fig. 3.6. Calculated desired bicycle torque and speed ...........................28

Fig. 3.7. Calculated E-bike torque components for one velocity cycle. ...............29

Fig. 3.8. Calculated E-bike power components for one velocity cycle. ...............29

Fig. 3.9. Torque-speed envelope and operating points on the velocity cycle. .............30

Fig. 3.10. Design process logic flow diagram. ................................ 32

Fig. 3.11. Torque performance comparison for different rotor pole arcs. ...............38 
M.A.Sc. Thesis - Jianing Lin; McMaster University - Mechanical Engineering.

Fig. 3.12. Physical view of SRM concentrated winding. .........................40

Fig. 3.13. Winding and energizing sequence comparison. .........................41

Fig. 3.14. Designed SRM. ................................................42

Fig. 3.15. Flux linkage versus current comparison at aligned and unaligned rotor commutation positions. ...................................................... 43

Fig. 3.16. Total torque comparison between 2D and 3D models. ...................45

Fig. 3.17. Flux path for unaligned and aligned commutation positions. ..............45

Fig. 3.18. Flux linkage versus position for different currents. .....................47

Fig. 3.19. Single phase torque versus position for different currents. ...............47

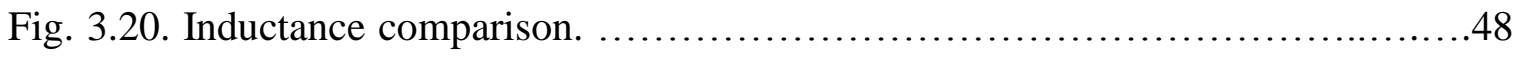

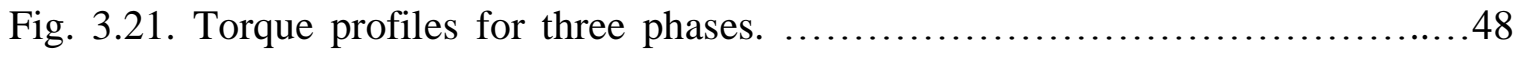

Fig. 3.22. Currents energizing sequence for the three phases. ......................49

Fig. 3.23. Total torque comparison for NSNSNS and NNNSSS. ..................50

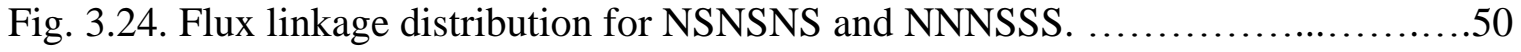

Fig. 4.1. Classic control diagram for SRM. ................................... 54

Fig. 4.2. Torque sharing function of three phases. ..............................58

Fig. 4.3. SRM modeling in Matlab/Simulink. .................................60

Fig. 4.4. Angular position control diagram in Matlab/Simulink. .....................61

Fig. 4.5. Starting ability with different turn-on and turn-off angles. ..................62

Fig. 4.6. Torque ripple at rated speed with different turn/off angles. .................63

Fig. 4.7. Current and torque profile with turn-on angle of -1 degree and turn-off of 14

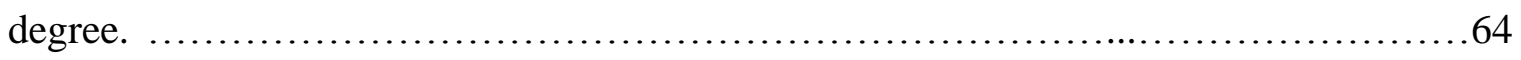


M.A.Sc. Thesis - Jianing Lin; McMaster University - Mechanical Engineering.

Fig. 4.8. Current and torque profile at rated speed with turn-on/off of -1 and 14 deg.....65 Fig. 4.9. Current and torque profile during accelerating with turn-on/off of -1 and 14

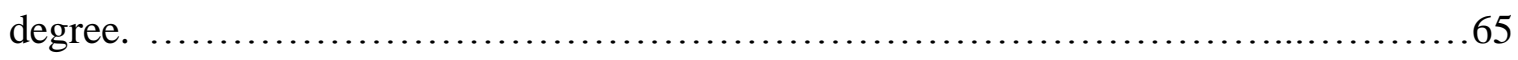

Fig. 4.10. Torque ripple at lower speed range with different turn/off angles...........66 Fig. 4.11. Current and torque profile at lower speed range with turn-on/off of 0 and 14 degrees.................................................................. 67

Fig. 4.12. Torque sharing function control diagram. .............................68

Fig. 4.13. Starting torque with TSF control. ................................. 70

Fig. 4.14. Torque ripple at lower speed range with TSF. .......................... 71

Fig. 4.15. Comparison of current and torque profiles with smaller overlap angle but different turn-on angles. ................................................ 72 Fig. 4.16. Comparison of current and torque profiles with larger overlap angle but

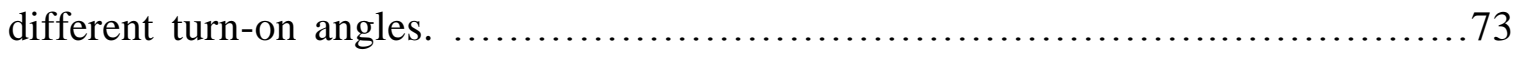
Fig.4.17. Current and torque profile with turn-on/overlap of 0 and 6 degrees for TSF control.............................................................. 73

Fig. 4.18. Starting torque curves with TSF control. ........................... 74

Fig. 4.19. Current and torque profile at rated speed with TSF. ..................... 74

Fig. 5.1. Coupling ways of thermal finite element analysis. ........................77

Fig. 5.2. Copper losses calculation for a typical velocity cycle. $\ldots \ldots \ldots \ldots \ldots \ldots \ldots \ldots . \ldots 8$

Fig. 5.3. Copper loss variation for one velocity cycle. .......................... 80

Fig. 5.4. Iron losses variation for one velocity cycle. ........................... 82

Fig. 5.5. Output power, copper and iron losses variation for one velocity cycle..........82 
M.A.Sc. Thesis - Jianing Lin; McMaster University - Mechanical Engineering.

Fig. 5.6. Efficiency variation for one velocity cycle. .............................. 83

Fig. 5.7. Thermal connection between different parts. ..............................84

Fig. 5.8. Temperature variation at rated operation condition. ........................85

Fig. 5.9. Temperature distribution at rated operation condition. ....................85

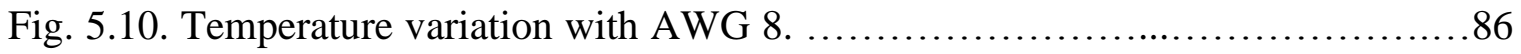

Fig. 5.11. Temperature variation from average loss of velocity cycle. .................86

Fig. 6.1. Expansion view of the designed motor in 3D drawing. $\ldots \ldots \ldots \ldots \ldots \ldots \ldots \ldots . \ldots 8$

Fig. 6.2. Comparison of the original stator and the design with wedge tips. .............89

Fig. 6.3. Comparison of the original design, the final design with wedge tips. ..........90

Fig. 6.4. Flux distribution on the final stator design with wedge tips. ................91

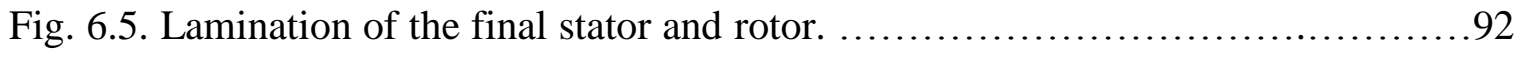

Fig. 6.6. 3D Shaft construction. ...........................................93

Fig. 6.7. Physical view of the stator on the shaft after winding. .................... 94

Fig. 6.8. 3D assembly view with physical parts below. ..............................96

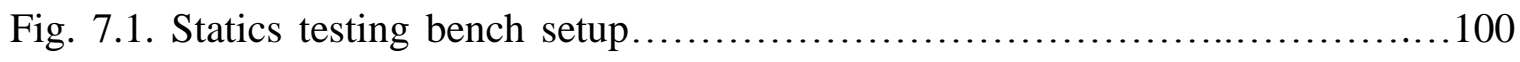

Fig. 7.2. Flux linkage curves at different aligned positions. ...................... 101

Fig. 7.3. Flux linkage curves comparison among three phases. ....................101

Fig. 7.4. Flux linkage profile comparison of the 2D FEA and experiment. ............103

Fig. 7.5. Torque profile comparison between 2D FEA and testing. .................. 103

Fig. 7.6. Flux linkage comparison between FEA with/without wedge tip and testing.104

Fig. 7.7. Flux linkage comparison between 3D FEA and testing. ..................... 105

Fig. 7.8. Torque profile comparison between 3D FEA and testing. ..................106 
M.A.Sc. Thesis - Jianing Lin; McMaster University - Mechanical Engineering.

Fig. 7.9. Equivalent circuit for flux linkage calculation. ......................... 107

Fig. 7.10. Dynamics testing bench. ........................................ 108

Fig. 7.11. Current and voltage at rated speed for no-load operation. .................109

Fig. 7.12. Energy curve of no-load operation. ............................... 110

Fig. 7.13. Energy curve comparison for different turn-off but constant turn-on angles..111

Fig. 7.14. Current profile with turn-on of 0 and turn-off of 16 degree. ...............111

Fig. 7.15. Energy curve comparison for different turn-on but constant turn-off angles..112

Fig. 7.16. Machine's speed-power curve. ....................................113

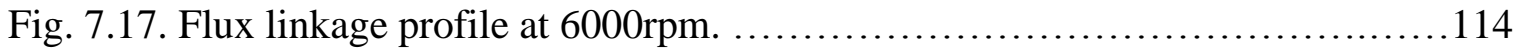


M.A.Sc. Thesis - Jianing Lin; McMaster University - Mechanical Engineering.

\section{LIST OF TABLES}

Table 1.1. Cost comparison between the Auto car and E-bike.........................2

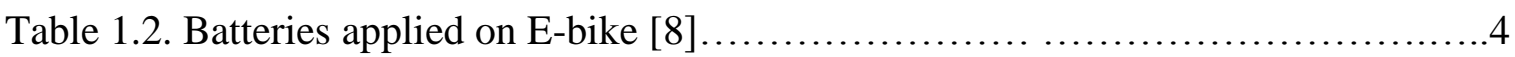

Table 1.3. Evaluation of brushed DC, PMBLDC and SRM for E-bike [18]-[20]...........7

Table 3.1 Bike kinetic model parameters and values [38] ............................24

Table 3.2 Motor kinetic model parameters and values...........................24

Table 3.3 Restrictions of the designed SRM....................................... 31

Table 3.4 Loss comparison for different stator pole arcs.......................... 37

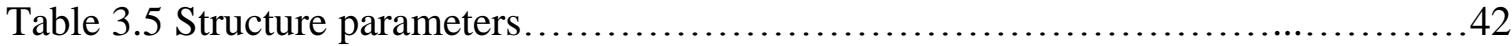

Table 3.6 Performance of the 6-10 external rotor SRM............................42

Table 3.7 Inductance comparison between FEA and SPEED $\ldots \ldots \ldots \ldots \ldots \ldots \ldots \ldots \ldots . \ldots 4$

Table 5.1. Losses and efficiency at rated condition.............................. 78

Table 5.2. Losses and efficiency comparison for different winding. .................. 79

Table 5.3. Average losses for one velocity cycle. .................................83

Table 6.1. Comparison of the origin stator design and the final design with wedge tips..90

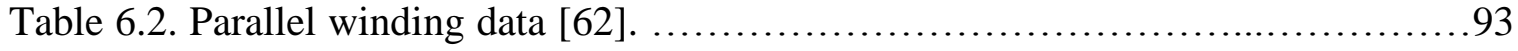

Table 6.3. Mechanical data comparison for the two shrink-fit parts [63],[64].........95

Table 7.1 Prototype machine structure parameters $\ldots \ldots \ldots \ldots \ldots \ldots \ldots \ldots \ldots \ldots \ldots \ldots . \ldots 9$

Table 7.2. Output power comparison for different turn-off but constant turn-on angles.111

Table 7.3. Performance with turn-on of -3 , and turn-off of 14 degree AP control. .....113 
M.A.Sc. Thesis - Jianing Lin; McMaster University - Mechanical Engineering.

\section{Chapter 1 Introduction}

Over the past decades, there has been an increasing personal transportation market, and bicycles are highly popular, especially in China, Europe, Japan and North America. However, bicycles are confronted with problem especially when compared with auto cars. People have to give up this choice when the travel distance is long and the road situation is bad. The solution proposes the combination of the conventional manually powered bicycle and the electric, or electric assisted, driving system, where an electric motor is applied on regular bicycle frame. Then the electric bicycle (E-bike) comes out.

The E-bike is gaining the world's popularity and capturing an increasing market share from the bicycle and auto car markets. For example, it has been reported that the population of E-bikes in China exceeded 140 million in 2012 [1]. In the same year, about 700,000 E-bikes were sold in Western Europe, nearly half of which were in Germany [2].

\subsection{Evaluation of E-bike}

As an environmentally-friendly mode of personal transportation, the E-bike also has the advantage of saving rider travel cost considerably. With one full charge, a common commercial E-bike can travel a distance of about 20-30 km [3], which will save about CAD $\$ 3.5$ per charge or more than CAD $\$ 1500$ per year, considering only gasoline costs as reported in [4]. Since the cost can be changed with different situation, some assumptions are made. According to Statistics Canada's data for 2008 [5], the average daily travel distance per vehicle is about $44 \mathrm{~km}$. And gas price is chosen at 120 cents per litre while a 
M.A.Sc. Thesis - Jianing Lin; McMaster University - Mechanical Engineering.

vehicle MPG of 25 is chosen [6].

Furthermore, these cost differences will increase if the automotive purchasing, maintenance, license and other fees are considered. A typical comparison between auto cars and E-bikes is shown in Table 1.

Table 1.1. Cost comparison between the Auto Car and E-bike.

\begin{tabular}{l|c|c}
\hline & Auto Car & New E-bike \\
\hline Purchase price & CAD $\$ 25,000$ & CAD\$2,000 \\
\hline license and registration & CAD $\$ 50$ & None \\
\hline Insurance & CAD\$2,000/year & None \\
\hline Sticker & CAD $\$ 200 /$ year & None \\
\hline Maintenance & CAD $\$ 500 /$ year & CAD $\$ 150 /$ year \\
\hline
\end{tabular}

The first E-bike came out around the 1980s. And now it's facing a new boom [7], with different kinds of E-bike available in the market. It is easy for a customer to buy a commercial prebuilt E-bike or to buy a conversion kit to adapt a regular bike to an E-bike.

Although the E-bike market expands rapidly, there are no uniform standards. For example, E-bike is also called electric, pedal-electric, power-assist or electric-motorpower bicycle, etc. while power-assist bicycles sometimes include engine-assist and there are also no-pedal E-bikes, which use motor power only [3]. However, in this thesis the term of E-bike is used to describe a bicycle equipped with an electric machine, battery and other auxiliaries, in which human pedal and electric machine power are both used to move the bicycle. 
M.A.Sc. Thesis - Jianing Lin; McMaster University - Mechanical Engineering.

There are some criteria used to classify E-bikes: they can be divided into city E-bikes and mountain E-bikes, distance E-bikes and speedy E-bikes based on operation condition and can also be classified according to its performance such as power, speed and torque. More often, they are classified according to electric machine location, type, battery type, assembly type, control method, as well as type of power assist. For the power assist type, full-assist and partial-assist are options, while partial-assist bicycle refers to those whose electric machine works only when the rider provides pedal power. These are common in Japan. The full-assist bicycle is more widely applied, including rider pedaling only mode, electric machine only mode and pedal-electric machine working together mode. The Ebike's power train can be parallel or serial, when human pedal works together with the electric machine. A typical parallel power flow cycle is illustrated in Fig.1.1. Normally parallel cycle has higher efficiency and is thus more widely used on E-bikes.

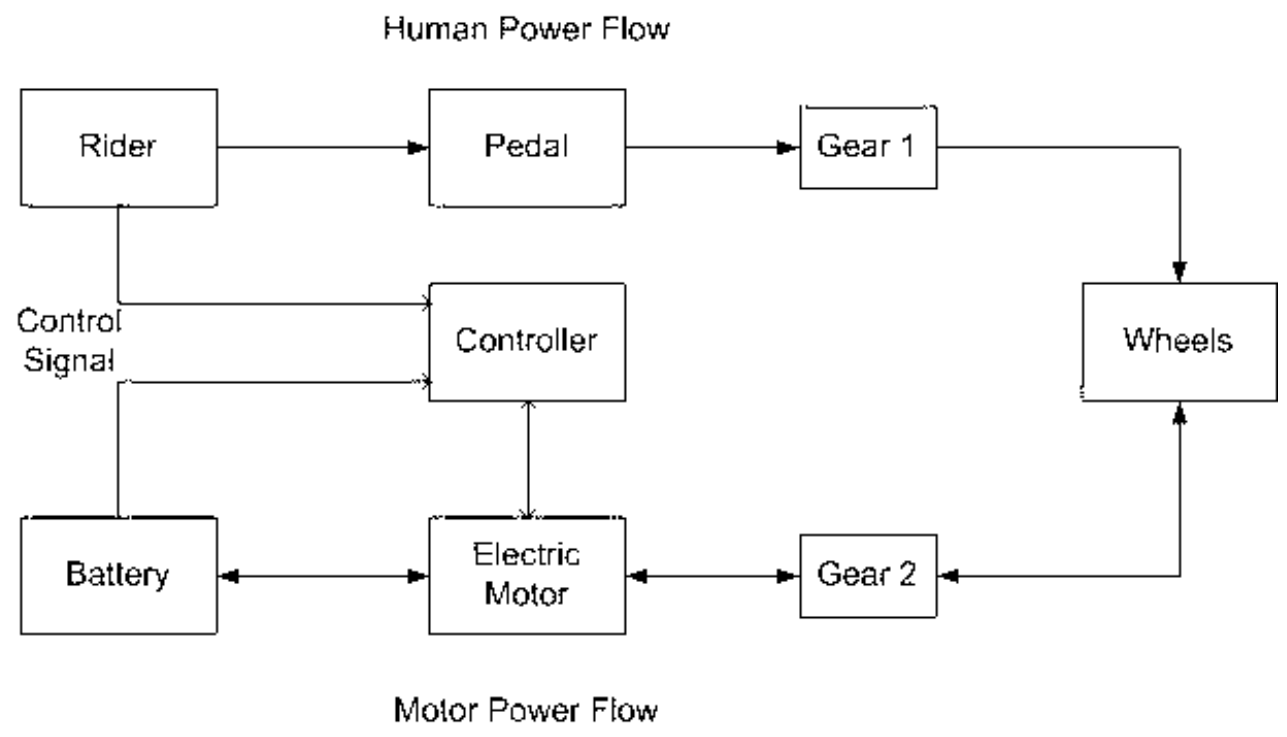

Fig. 1.1. A typical parallel hybrid schematic diagram of E-bike. 
M.A.Sc. Thesis - Jianing Lin; McMaster University - Mechanical Engineering.

The on-board energy source is typically an electro-chemical battery and this important technology is the one that has slowed down the development of the E-bike. However, with its continued development, there become more and more opportunities for E-bike, on the grounds of cost, reliability and lifetime. There are kinds of electro-chemical battery available for the E-bike market, such as lead acid (Pb-acid), nickel metal-hydride (NiMH) and lithium-ion (Li-ion), etc. The comparison of these technologies is made in Table 1.2, where Li-ion is now drawing more attention in the E-bike industry because of its high energy density, long lifetime and reasonable cost base.

Table 1.2. Batteries applied on E-bike [8].

\begin{tabular}{l|c|c|c}
\hline & Pb-acid & NiMH & Li-ion \\
\hline $\begin{array}{l}\text { Mass-Energy } \\
\text { Density (Wh/kg) }\end{array}$ & $20-30$ & $50-60$ & $100+$ \\
\hline Lifetime (Hours) & $300 \pm$ & 2000 & $600+$ \\
\hline Stability & Stable & Stable & $\begin{array}{c}\text { Supersaturating } \\
\text { cause explosion }\end{array}$ \\
\hline Charging Speed & Slow & $\begin{array}{c}15 \text { min. charging can reach } \\
60 \% \text { normal battery } \\
\text { capacity. }\end{array}$ & Slow \\
\hline Memory Effect & Y & Y & N \\
\hline Cost & Low & $\begin{array}{c}\text { More expensive than } \\
\text { Li-ion }\end{array}$ & $\begin{array}{c}\text { Capital cost about 3 } \\
\text { times of Pb-acid }\end{array}$ \\
\hline $\begin{array}{l}\text { Environmental } \\
\text { concerns }\end{array}$ & $\begin{array}{c}\text { Lead } \\
\text { pollution }\end{array}$ & Mild pollution & Lithium pollution \\
\hline
\end{tabular}

\subsection{Electric Motors on E-bike}

As mentioned before, there is a big E-bike market especially in China, Europe and North America. However, there is still a significant challenge for E-bike development with regard to the trade-off between E-bike performance and the price. The key difficulty 
M.A.Sc. Thesis - Jianing Lin; McMaster University - Mechanical Engineering.

for E-bike manufacturers in Europe and North America is the competition from Chinese E-bike market. Designing a high performance and lower cost E-bike is the main objective of many E-bike manufacturers, and involves the design of a well-qualified powertrains, including electric propulsion machine, electronic controller, battery pack, and charger. Thus, the electric machine is an especially important component that influences overall E-bike performance.

For electric propulsion applications, electric machines with high torque and power density, high efficiency, high reliability and robustness are required. Moreover, reducing cost is of significant importance to the development of electric vehicles, particularly for the E-bike industry.

For electric vehicles, induction machine (IM) and permanent-magnet machine are highly dominant, while the number of brushed DC motors are decreasing, and brushless DC (BLDC) machine is gaining increasing interest [9], [10]. Moreover, with appropriate design and optimal control, SRM is potential for performance superior to BLDC's and IM's [11].

A review of the E-bike market shows that the electric propulsion machines are predominantly brushed DC and brushless DC (BLDC) machines [12], [13]. An inverter driven linear induction motor was proposed by K. Watanabe in his paper [14]. There was also a sensor-less permanent magnet AC motor studied by Reiko Raute in 2005 [15]. But most of them were forgotten because of their high development cost or poor performance on E-bike. In addition, IM has another problem which stunts its growth for EV or E-bike. 
M.A.Sc. Thesis - Jianing Lin; McMaster University - Mechanical Engineering.

Since IM owns a very limited constant power region, it suffers a very limited speed range. Therefore, brushed DC and BLDC are more popular on E-bikes. For example, Heinzmann was one of the original hub brushed DC motor manufacturers many years ago, who designed the motor mounted on a fork bracket, while Sanyo and Panasonic once produced BLDC motors for E-bike [16]. Now BionX is one of the biggest E-bike manufacturers in Canada which offers various kinds of brushless DC motors for electric bicycles [17].

Compared with BLDC, brushed DC has a simpler structure and a lower cost. But it is hard to maintain due to the commutator, and has low efficiency. Although its efficiency can be improved by its direct driving, this also leads to the problem of big volume for enough torque performance.

Comparatively, BLDC has more advantages: 1. Without the commutators, brushless motor has a longer lifetime and no maintenance problem. 2. Taking the winding out of the rotor also increases brushless DC motor's efficiency. 3. In addition, brushless motor also has higher torque and power density, etc [18]. However, the permanent magnet material increases the BLDC motor cost, particularly in resent years due to the concerns over the demand for rare earth materials.

To illustrate the above discussion, a comparison is made between brushed DC, PMBLDC and SRM by using weight points as presented in Table 1.3. To address these problems, a high-quality and low-cost SRM is studied and reported in this thesis. Noted in Table 1.3., the maximum points indicate the machine best meets the requirement. Despite 
M.A.Sc. Thesis - Jianing Lin; McMaster University - Mechanical Engineering.

the fact that PMBLDC and SRM get the same points, SRM is finally chosen because of its low cost, since capital impact is usually one of the most important selection criteria for E-bikes.

Table 1.3. Evaluation of brushed DC, PMBLDC and SRM for E-bike applications [18]$[20]$.

\begin{tabular}{l|c|c|c}
\hline & Brushed DC & PMBLDC & SRM \\
\hline Power Density & 2.5 & 5 & 3.5 \\
\hline Overload & 3 & 3.5 & 4 \\
\hline Efficiency & 2.5 & 5 & 3.5 \\
\hline High Speed Range & 2.5 & 2.5 & 4.5 \\
\hline Controllability & 5 & 4 & 5 \\
\hline Noise & 4 & 4.5 & 3 \\
\hline Torque Ripple & 3.5 & 4 & 2 \\
\hline Size and Weight & 2.5 & 3.5 & 4.5 \\
\hline Ruggedness & 3.5 & 4 & 4.5 \\
\hline Maintenance & 2 & 5 & 4 \\
\hline Maturity & 5 & 8 & 10 \\
\hline Cost & 10 & 52 & 52 \\
\hline Total & 46 & & \\
\hline
\end{tabular}

\subsection{Switched Reluctance Motor on E-bike}

SRM is an electric motor that operates by chance in magnetic circuit that results in a reluctance torque. The SRM was first documented in $1837-1840$ by a patent submitted by B. D. Bedford [21]. However, this machine required mechanical switches that were 
M.A.Sc. Thesis - Jianing Lin; McMaster University - Mechanical Engineering.

inefficient and with the appearance of DC motors in the second half of the 19th century, SRM lost all attention. This remained the case until the advent of modern power electronics and development restarted with the publication of Lawrehson's paper of 1980 $[22]$.

During its development history, many SRM topologies have been studied. There are some common structures of SRMs, such as the: single-phase 8/8 structure motor [23]; two-phase 4/2 structure motor [24], [25]; three-phase 6/4 structure motor [26]; threephase 6/8 structure motor [27]; three-phase 12/8 structure motor [19]; and four-phase 8/6 structure motor [28], although this list is not exhaustive. Different structures have different characteristics. To decide a certain structure for a special application usually requires a number of steps to choose and optimize the design. A detailed design process will be conducted in Chapter 3 for this E-bike application.

As for E-bikes, there are already some researchers like H. Chen, who have applied SRM on electric bicycles. A $6 / 4$ and 12/8 SRM as well as the control for E-bikes have been studied in [29]. However, these motors are all low power, within 200W, while their performance and efficiency don't meet current E-bike requirements. Moreover, there is also very limited information about the design of these SRMs for E-bikes. Thus, this thesis presents a systematical study on SRM design for electric bicycles.

The SRM has many attributes for EV drives. Among these advantages, the machine simple structure and low cost make it really attractive for E-bikes. However, its torque ripple and noise constrain its uptake, especially for low speed and quiet applications. 
M.A.Sc. Thesis - Jianing Lin; McMaster University - Mechanical Engineering.

However, since SRM research is limited to the latest three decades, there are enough reasons to believe that these weaknesses can be solved in the future, especially with the development of improved power electronic control and novel electromagnetic design structures. There is already some published evidence that SRMs can compete with PM motors. From the perspective of control, theoretically, even a 6/4 SRM can be controlled to have zero torque ripple, as discussed in [30]. As for the design, some particular structures also have the feature of reducing torque ripple and noise significantly. For example, a multilayer SRM was designed and reported in [31]. In papers [32] and [33], the SRM is designed to have the same outer diameter and axial length of a benchmark IPMSM employed in the second generation of Toyota Prius 2003. Experiment results from [32] and [33] show that the SRM efficiency, torque and power- speed range are very competitive over the IPMSM. In [34], a 60kW SRM is designed, which has performance that compete with the Toyota Prius third generation IPMSM.

Therefore, given the above publications, an optimally designed and well controlled SRM could be technically comparable with a PMBLM and thus suitable to be applied on E-bikes, hence the subject of this thesis study.

\subsection{Conclusion}

Compared with conventional bike, E-bikes are suitable for longer distance and harder road condition. At the same time, this zeron-emssion personal transportation saves a considerable cost compared with auto car. SRM's advantages of lower cost, higher power 
M.A.Sc. Thesis - Jianing Lin; McMaster University - Mechanical Engineering.

density, good ruggedness make it quite attractive for EV application, which is already approved by publishes. However, for the E-bike application, there is less study, especially for higher performance E-bikes. Hence, this thesis is going to present a novel SRM design, together with its control and thermal analysis for the E-bike application. 
M.A.Sc. Thesis - Jianing Lin; McMaster University - Mechanical Engineering.

\section{Chapter 2 Fundamental of SRM Design and its Control}

\subsection{Operation Principle of SRM}

SRM's operation is relatively simple. When the stator coils are excited, the machine has the tendency to move from a position with a larger magnetic reluctance to a position with a smaller one, i.e. the rotor moves to minimize the magnetic circuit reluctance.

Since the machine soft magnetic material has a much smaller magnetic reluctance than that of air, if phase $\mathrm{A}$ is energize, the rotor pole will move from the unaligned commutation position as shown in Fig. 2.1 (a), to the aligned commutation position, Fig. 2.1 (b). After that, phase A is de-energized, while at the same time, phase B is energized, the rotor poles will thus continue to move from the unaligned position of phase B, Fig. 2.1 (c), to the aligned position of phase B, Fig. 2.1 (d). Similarly for phase C, hence the rotor will rotate counter clockwise. However, if the phases are energized BAC instead of ABC, the rotor will rotate clockwise.

Consideration of this basic operation highlights that the operating direction has nothing to do with the current polarity. Direction of rotation is determined by the sequence of the energized phases, and the frequency of this switching sequence dictates the speed of the rotor. 
M.A.Sc. Thesis - Jianing Lin; McMaster University - Mechanical Engineering.
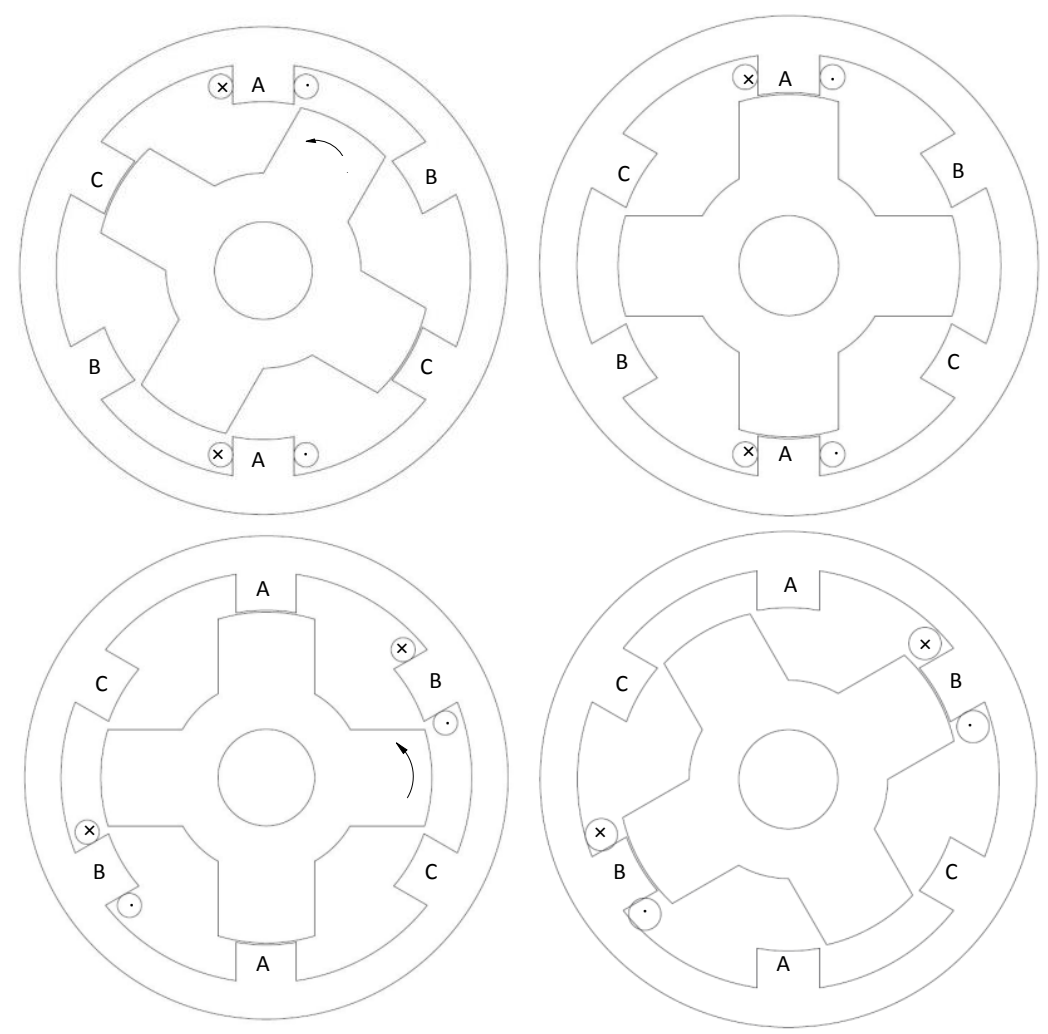

Fig. 2.1. SRM's operating principle.

\subsection{Mathematic Model of SRM}

The SRM operation is an electromagnetic process, hence it follows that the laws of electromagnetic induction, Kirchhoff's current law and the law of conservation of energy. Based on these laws, the SRM mathematics model is developed, as illustrated schematically by the simplified equivalent circuit of Fig. 2.2. It also shows the energy conservation process of the SRM. The left part represents the electrical circuit of the SRM, while the right part indicates the mechanical operation process, with the middle part detailing the electromagnetic transfer process. 
M.A.Sc. Thesis - Jianing Lin; McMaster University - Mechanical Engineering.

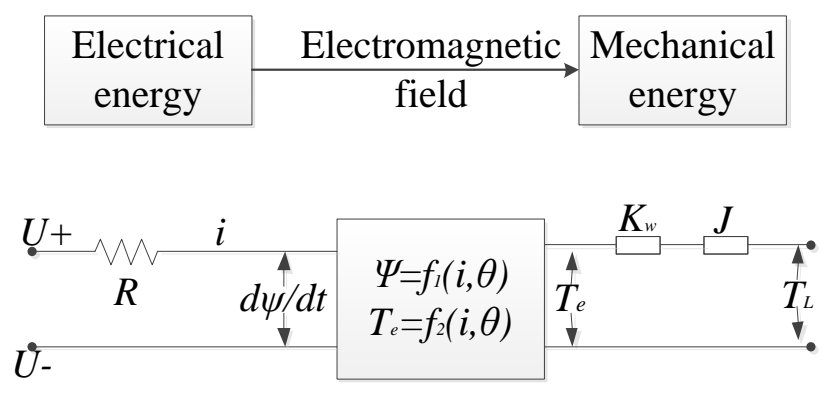

Fig. 2.2. Simulation diagram of SRM.

Firstly, the voltage equation of each phase of the SRM can be written as:

$$
u_{k}=i_{k} R_{k}+\frac{d \psi_{k}}{d t}
$$

where $u_{k}, i_{k}, R_{k}$ and $\psi_{k}$ are the DC supply voltage, phase current, electrical phase resistance and phase flux-linkage of phase $k$.

Equation (2-1) shows that the voltage drop has two components: one due to the electrical resistance of the phase winding, while the other due to the flux-linkage variation in the phase winding. The phase coil flux-linkage is produced not only by coil selfinduction, but also by mutual induction with other coils.

However, the analysis here only considers the situation of one-phase energizing, and thus the mutual induction can be ignored. To simplify the process, flux-leakage is not considered. Since $\psi_{k}=i_{k} L_{k}$, equation (2-2) can be derived.

$$
\frac{d \psi_{k}}{d t}=L_{k} \frac{\partial i_{k}}{\partial t}+i_{k} \frac{\partial L_{k}}{\partial t}
$$


M.A.Sc. Thesis - Jianing Lin; McMaster University - Mechanical Engineering.

The voltage equation (2-1) can be rewritten as equation (2-3), by mathematical decomposition:

$$
u_{k}=i_{k} R_{k}+\left(L_{k}+\frac{1}{2} i_{k} \frac{\partial L_{k}}{\partial i_{k}}\right) \frac{\partial i_{k}}{\partial t}+\frac{1}{2} i_{k} \frac{\partial L_{k}}{\partial \theta} w
$$

where $L_{k}$ is the induction of phase $k$. Equation (2-3) shows that the flux variation is caused by two mechanisms: one is the current changing in the coil and the other is the rotation of the rotor. The input voltage is thus finally defined by three parts: the resistive voltage drop, the inductive voltage drop and the back-EMF.

When an electro-magnetic machine works as an electric motor, it converts electrical energy to mechanical energy. To simplify the process, description assume that the whole process is within the SRM unsaturated condition. Then flux-linkage-current characteristic of the process switching conditions shown in Fig. 2.1 (a) and Fig. 2.1 (b) can be represented on the system flux-linkage versus phase current diagram, as shown in Fig. 2.3.

For motor operation, part of the electrical input energy $W_{e}$ is stored in the phase magnetic circuit as $W_{f}$ and the other part is converted to mechanical energy as $W_{m}$ [35].

Thus, the electrical input energy can be expressed by:

$$
W_{e}=W_{f}+W_{m}
$$

For the assumed unsaturated condition, the energy stored in the phase coils can be expressed by: 
M.A.Sc. Thesis - Jianing Lin; McMaster University - Mechanical Engineering.

$$
W_{f}=\int i d \lambda=\int i d(i \cdot L)=\frac{1}{2} L \cdot i^{2}+\int i^{2} d L
$$

Where $\lambda$ is the phase coil flux- linkage, $L$ is the self inductance of the phase coils, and $i$ is the exciting current.

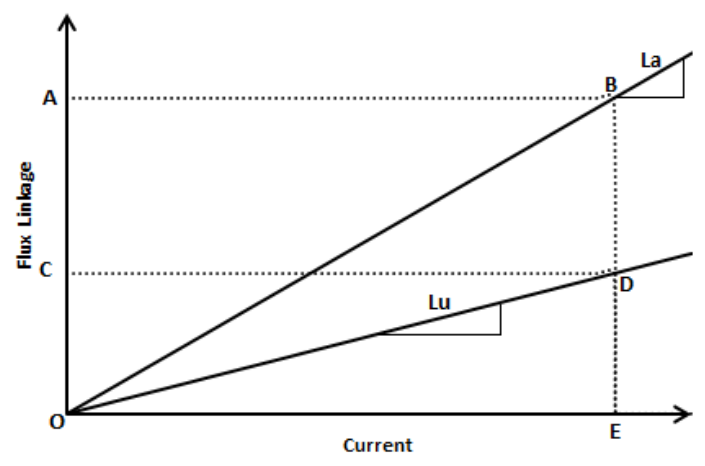

Fig. 2.3. Ideal flux-linkage versus phase current profiles at rotor aligned and unaligned commutation positions.

As the SRM rotates from the unaligned commutation position to the aligned commutation position with a constant current $i_{c}$, the motor state moves from point $\mathrm{D}$ to $\mathrm{B}$ in Fig. 2.3. Then, the second part of (2-5) can be solved as:

$$
\int i^{2} d L=i^{2} \cdot\left(L_{a}-L_{u}\right)=i_{c} \cdot\left(\lambda_{a}-\lambda_{u}\right)
$$

Where $L_{a}$ is the inductance value at the aligned commutation position, and $L_{u}$ the inductance value at unaligned commutation position.

During this same period, the electrical input energy changes from area OCDEO to area OABEO. The electrical input energy is related to the excitation and the flux-linkage at different positions, which can be expressed as [35]: 
M.A.Sc. Thesis - Jianing Lin; McMaster University - Mechanical Engineering.

$$
W_{e}=F_{c} \cdot\left(\phi_{a}-\phi_{u}\right)
$$

where $F_{c}=N \cdot i_{c}$ and $\phi=\frac{1}{N} \lambda$. The second part of the stored energy in (2-6) equals the electrical input energy in (2-7).

From (2-4)-(2-7), equation (2-8) is derived. This indicates the converted mechanical energy comes from the first part of the stored energy in coils.

$$
W_{m}=\frac{1}{2} L \cdot i^{2}
$$

For the motor energy system, the decreasing energy means an increasing output mechanical energy. Thus,

$$
W_{m}=\frac{1}{2} L \cdot i^{2}=T_{e} \cdot \delta \theta
$$

If phase current is assumed constant, the electromagnetic torque for each phase can be derived as:

$$
T_{e}=\frac{1}{2} \cdot \frac{d L}{d \theta} \cdot i^{2}
$$

The electrical energy is converted to mechanical energy via the changing electromagnetic field.

As the excited phases switch sequentially, energy conversion will be continuous and the rotor will rotate as required. From (2-10), it can be seen that the SRM torque is not related to the polarity of phase current; and direction and speed of rotation are related to the sequence of the excited phases and the switching frequency. 
M.A.Sc. Thesis - Jianing Lin; McMaster University - Mechanical Engineering.

To increase the area for energy conversion, SRM are operated outside the linear magnetic characteristics and therefore magnetic saturation must be considered. This can be a problem for machine design and control.

The machine electromagnetic torque is used to overcome friction torques, provides machine acceleration torque and the required external load torque, as represented in equation (2-11):

$$
T_{e}=J \frac{d^{2} \theta}{d t^{2}}+K_{w} \frac{d \theta}{d t}+T_{L}
$$

where $J$ is the machine rotor and system moment of inertia, $K_{w}$ is the machine viscous friction constant and $T_{L}$ is the torque applied to the rotor by an external load.

\subsection{SRM Control}

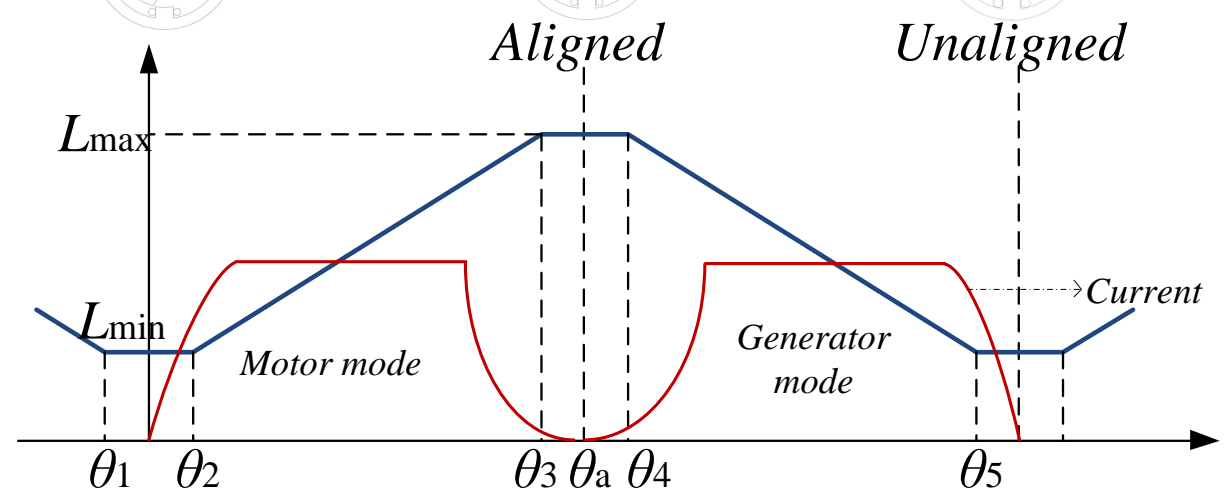

Fig. 2.4. Phase inductance characteristics versus rotor position, with motor and generator modes showing. 
M.A.Sc. Thesis - Jianing Lin; McMaster University - Mechanical Engineering.

From equation (2-10), a net positive motoring toque is achieved when the phase is excited when the inductance increases. As discussed previously, the cyclic switching characteristic of the SRM, where all phase current profiles repeat at every rotor pole pitch, allows analysis of only one rotor pole pitch to represent complete rotation. The nonlinear characteristic introduces complexity in analysis, thus a linear, simplified model of phase inductance variation can be applied to explain the torque generation process of the SRM, as illustrated in Fig. 2.4.

From Fig. 2.4, the following relationships hold:

$$
\begin{aligned}
& \theta_{2}=\frac{1}{2}\left[\frac{2 \pi}{P_{r}}-\left(\beta_{s}+\beta_{r}\right)\right] \\
& \theta_{3}=\theta_{2}+\beta_{s} \\
& \theta_{4}=\theta_{3}+\left(\beta_{r}-\beta_{s}\right) \\
& \theta_{5}=\theta_{4}+\beta_{s}
\end{aligned}
$$

where $\beta_{s}, \beta_{r}$ are the stator pole arc and rotor pole arc respectively and $P_{r}$ is the rotor pole number.

As Fig. 2.4 shows, if the phase current is excited at the inductance increasing positions, the machine operates at a motor mode, but a generator mode if at the decreasing inductance range. To avoid negative torque generation, the turn-on and turn-off angles have to be specified.

For this nonlinear model, the inductance profile of which is not only a function of the rotor position but also of current. It requires more complex analysis to figure out the right turn-on and turn-off. This analysis is called SRM's control strategies. 
M.A.Sc. Thesis - Jianing Lin; McMaster University - Mechanical Engineering.

A controller has motor's dynamic parameter as input, using its control strategy to generate signals to control the inverter to turn on or turn off under a certain condition. To realize the E-bike's powertrain system, SRM's structure design, along with its control strategies, will be studied to optimize the whole system in this paper.

\subsection{Converter Configuration}

Different control strategies can be used to generate phase energizing sequence signals. To apply these sequences to a SRM requires an electronic commutation connected with a DC power source, which is also called converter.

For the power converters, a most commonly used classical asymmetric 2-switch/phase converter is used in this paper. Asymmetric bridge converter has the advantage of simple concept, flexibility in control, as well as better faults tolerance. However, it also requires larger number of switches. Fig. 2.5 shows the asymmetric bridge converter of one phase, and there are two switches for each phase, an upper one and a lower one. In this paper, the upper switch works with hysteresis current control to keep the current to be within a limitation, while the lower switches is controlled by the other control strategy, which will indicates when the phase should be turned on and when it should be turned off. Besides the hysteresis current control, the other control can be angular position control or torque sharing control, which will be studied later for the designed SRM in this paper. These two control strategies will work together to achieve a smooth and high efficiency motor drive. 
M.A.Sc. Thesis - Jianing Lin; McMaster University - Mechanical Engineering.

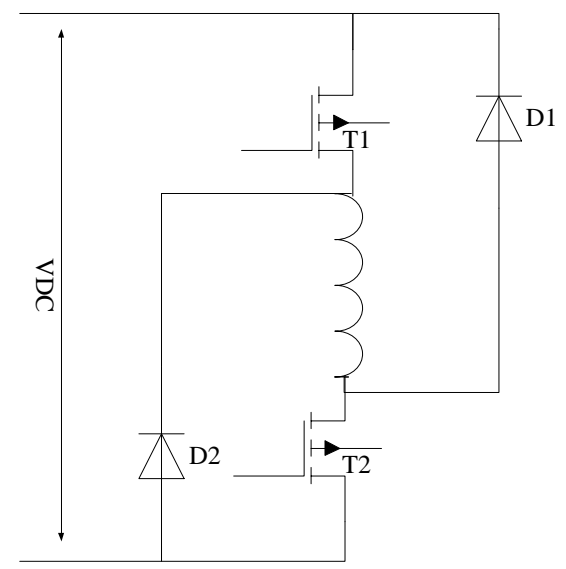

Fig. 2.5. Configuration of asymmetric bridge converter for single phase.

\subsection{Conclusion}

SRM's torque is mainly reluctance torque, which is under the minimum magnetic reluctance principle. This makes its structure simple and rigid. As its operating direction and speed are only determined by the sequence of the energized phases and the switching frequency, it also has higher fault tolerance than conventional machines. However, its operation principle and structure determine that SRM has to work in a deep magnetic saturated condition. This leads to a high nonlinearity, which definitely increases the design and control complexity of this machine. An basic asymmetric bridge converter structure is applied in this design. 
M.A.Sc. Thesis - Jianing Lin; McMaster University - Mechanical Engineering.

\section{Chapter 3 Design a SRM for Electric Bicycle}

Chapter 1 discuss the specific advantages of the SRM that make it a strong candidate for E-bike, while Chapter 2 has discussed basic operation of the machine. However, the SRM also has disadvantages of high torque ripple and resulting high acoustic. Thus, this is an important consideration for future design.

\subsection{Configuration of Electric Bicycle System}

To design a suitable E-bike SRM, the application requirements must be defined at the start of the design. In addition to the aforementioned requirements of high efficiency, high torque and power density and a reasonable speed range, a number of other specifications requirements were considered at the design stage:

The legislation in Ontario, Canada, limits the output power of E-bikes to be within 500 Watt and the maximum speed on the road must not exceed $32 \mathrm{~km} / \mathrm{h}$ with motor power alone [36]. The SRM maximum speed in this paper is chosen to be 500rpm and thus a reduction gear-box will be used to make its on-road speed match the legal limit in Ontario.

As a low speed vehicle, there is a stricter requirement on torque ripple for E-bike since this impact on rider comfort and hence product acceptance. More attention has to be paid to torque ripple reduction together with achieving the other design goals of high efficiency and advancing performance.

Another design constraint is the available power source for the vehicle which should be within an acceptable safe voltage range while having sufficient energy. A Samsung 48V 
M.A.Sc. Thesis - Jianing Lin; McMaster University - Mechanical Engineering.

10Ah lithium-manganese battery was chosen as the DC source. The maximum current of one cell is 35A, and this limits the maximum control current for the SRM.

As in-wheel construction is preferred for E-bike applications, an outer rotor structure is chosen for this study, since it has the potential for lighter weight and is ease of manufacture. A schematic of the integrated SRM and E-bike wheel is shown in Fig. 3.1. The outer diameter of the SRM is set to $220 \mathrm{~mm}$ and axial thickness to $25 \mathrm{~mm}$ to satisfy the in-wheel construction.

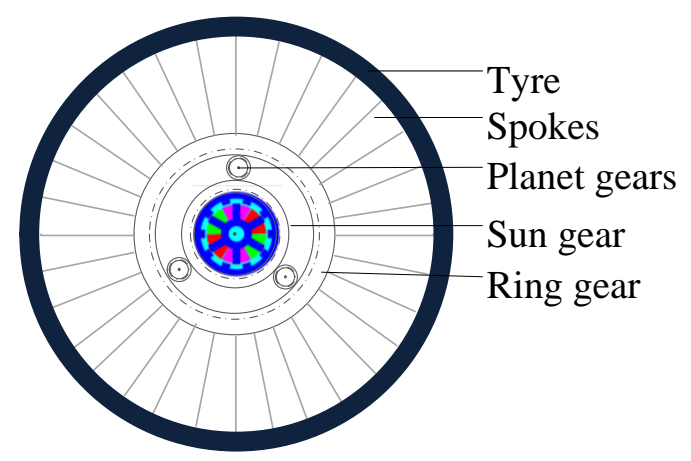

Fig. 3.1. Mechanical design for the in-wheel configuration.

\subsubsection{The road load and traction force requirements for the E-bike}

Besides the basic layout configuration information, the road load and traction force requirements have to be specified for the SRM design. Thus, kinematics are analyzed and used to calculate the E-bike force requirements, as shown in Fig. 3.2. 
M.A.Sc. Thesis - Jianing Lin; McMaster University - Mechanical Engineering.

The required force to propel the E-bike can be considered as 4 components: the force to accelerate or retard the vehicle mass $F_{a}$, the gravitational force $F_{g}$, air friction force $F_{a i r}$, and the wheel-to-road friction force $F_{r}$.

$$
\begin{aligned}
& F_{a}=M \cdot a(\mathrm{t}) \\
& F_{g}=M \cdot g \cdot \sin (\theta) \\
& F_{a i r}=\frac{1}{2} \rho \cdot M \cdot C_{D} \cdot A_{f} \cdot v(\mathrm{t})^{2} \\
& F_{r}=M \cdot g \cdot C_{r} \cdot \cos (\theta)
\end{aligned}
$$

where the parameters are as defined in Table $3.1, a$ is the acceleration of the E-bike. The mechanical system parameters are presented in Table 3.2.

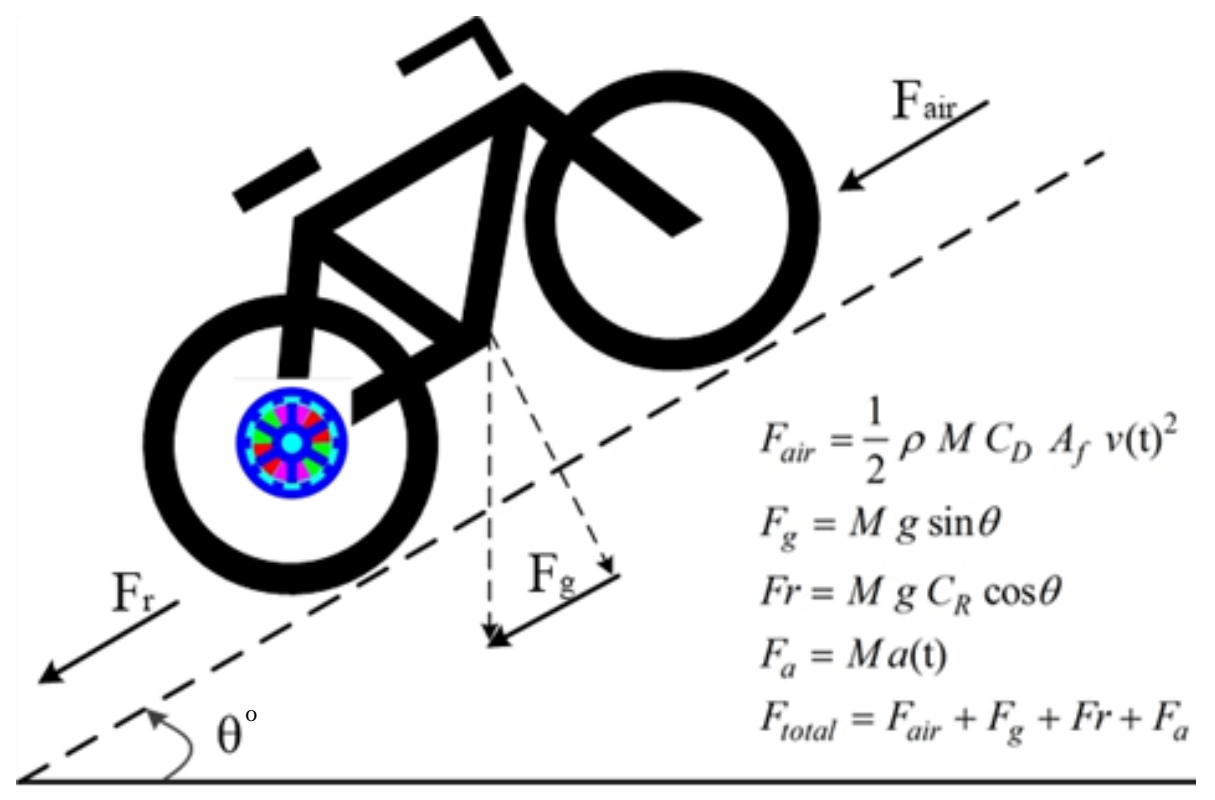

Fig. 3.2. Road Load and Traction Force Applied to the E-bike [37]. 
M.A.Sc. Thesis - Jianing Lin; McMaster University - Mechanical Engineering.

Table 3.1 Bike kinetic model parameters and values [38].

\begin{tabular}{l|c|c|c}
\hline \multicolumn{1}{c|}{ Items } & Parameters & Values & Units \\
\hline Mass of E-bike and Cyclist & $M$ & 100 & $\mathrm{~kg}$ \\
\hline Acceletarion due to gravity & $g$ & 9.81 & $\mathrm{~m} / \mathrm{s}^{2}$ \\
\hline Density of air & $\rho$ & 1.23 & $\mathrm{~kg} / \mathrm{m}^{3}$ \\
\hline Air resistance coefficient & $C_{D}$ & 0.5 & - \\
\hline Head Frontal Area (Cyclist) & $A_{f}$ & 0.5 & $\mathrm{~m}^{2}$ \\
\hline Tire friction coefficient & $C_{r}$ & 0.004 & - \\
\hline Gradient of the road & $\theta$ & 0 & $\mathrm{deg}$ \\
\hline
\end{tabular}

Table 3.2 Motor kinetic model parameters and values.

\begin{tabular}{l|c|c|c}
\hline \multicolumn{1}{c|}{ Items } & Parameters & Values & Units \\
\hline Frontal/rear wheel radius & $R$ & 0.33 & $\mathrm{~m}$ \\
\hline Moment of intertia of the rear wheel & $J_{w}$ & 0.0885 & $\mathrm{~kg} \mathrm{\textrm {m } ^ { 2 }}$ \\
\hline Moment of intertia of the motor & $J_{m}$ & 0.0254 & $\mathrm{~kg} \mathrm{\textrm {m } ^ { 2 }}$ \\
\hline Reduction gear ratio & $i_{\text {gear }}$ & 3 & - \\
\hline Motor efficiency & $\eta_{m}$ & 0.9 & per unit \\
\hline Chain efficiency & $\eta_{c}$ & 1 & per unit \\
\hline Gear efficiency & $\eta_{g}$ & 1 & per unit \\
\hline
\end{tabular}

Equations (3-1) to (3-4) are summed to yield the total load force required by the E-bike, from which the required E-bike total load torque at the wheels, $T_{w}$, can be obtained from equation (3-5):

$$
T_{w}=J_{w} \cdot \frac{d w_{w}}{d t}+d_{f} \cdot\left(R \cdot F_{a}+R \cdot F_{g}+R^{3} \cdot F_{a i r}+R \cdot F_{r} \cdot v(t)\right)
$$


M.A.Sc. Thesis - Jianing Lin; McMaster University - Mechanical Engineering.

where $\frac{d w_{w}}{d t}=\frac{a(t)}{R} ; d_{f}$ is a factor proportioning torque distribution between the front and rear axles. This is set to 1 , because only one motor is considered in this application. The total torque can be provided by the electric machine combined with the cyclist pedal input.

The equivalent desired motor torque $T_{d}$ is calculated by considering the wheel torque and gear coupling to the motor, as in equation (3-6):

$$
T_{d}=J_{m} \cdot \frac{d w_{m}}{d t}+\frac{1}{i_{\text {gear }} \cdot \eta_{g}} \cdot T_{w}
$$

where $\eta_{g}=1$ and $\frac{d w_{m}}{d t}=\frac{a(t) \cdot i_{\text {gear }}}{R}$. The equivalent E-bike motor power is also calculated in equation (3-7), which also has two sources, the electric motor, $P_{m}$, and the human pedal, $P_{p}$

$$
P_{d}=P_{m}+P_{p}=T_{m} \cdot w_{m}+P_{p}
$$

Considering the advantages and disadvantages of the front or rear wheel motor for the E-bike, the rear wheel drive has better traction, which is useful for hill climb and incline. Furthermore, because the rear frame dropouts (the wheel structural frame) are normally stronger than the front fork dropouts, the majority of system mass is better on the rear frame. Thus, for this study, the motor is placed on the rear wheel of the bike.

The motor output torque/power and human-pedal torque/power are assigned according to the logic flow chart illustrated in Fig. 3.3. The basic idea of this chart is that braking recharging is not considered, at least in this analysis. When the equivalent desired motor 
M.A.Sc. Thesis - Jianing Lin; McMaster University - Mechanical Engineering.

torque is negative, which relates to braking and speed decreasing, the motor output torque and power is set to be zero, and the mechanical brake and cyclist will take care of this process.

When the equivalent demanded motor torque is larger than the maximum output torque of the motor, the motor output torque is set to be the maximum value, and power checked. If power demand is bigger than maximum output power of $500 \mathrm{Watts}$, it is set to 500 Watts, and output torque is then reset to be $500 \mathrm{Watt} / \mathrm{speed}$. These remaining power and torque are then provided by the cyclist pedalling.

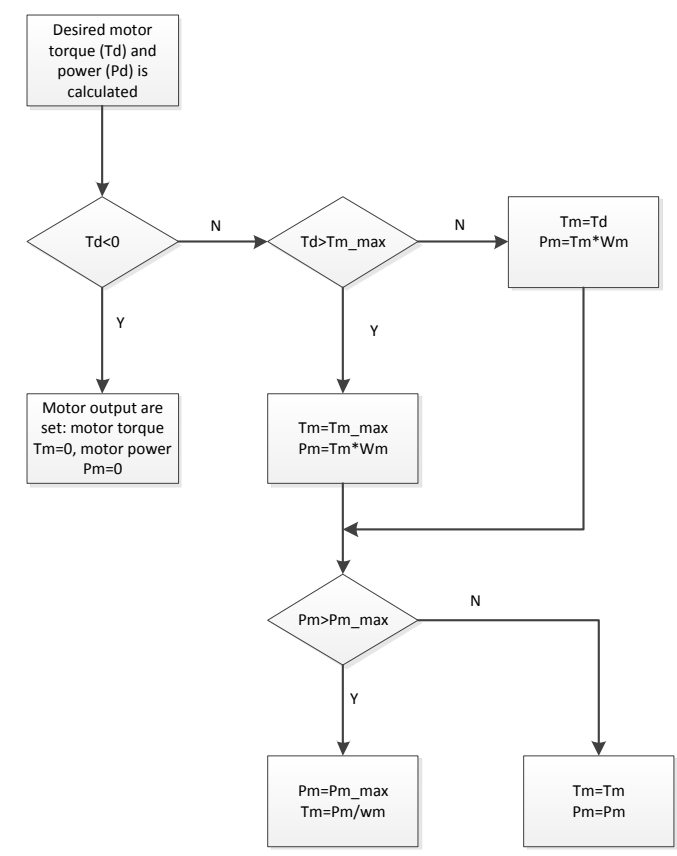

Fig. 3.3. Flow diagram showing human pedal and motor-output torque and power calculation logic. 
M.A.Sc. Thesis - Jianing Lin; McMaster University - Mechanical Engineering.

\subsubsection{The typical velocity profile of E-bike}

To analyze and compare a designed motor, its operating conditions are required for analysis and evaluation. However, since an equivalent driving cycle is not available for Canada, a European (Portuguese standard NP EN 1986-1) driving cycle [37] has been chosen as the reference for the machine design process, as illustrated in Fig 3.4.

As previously discussed, the maximum speed for an E-bike on road in Ontario, Canada is $32 \mathrm{~km} / \mathrm{h}=8.89 \mathrm{~m} / \mathrm{s}$. The rated speed of this designed motor is 500rpm. With a reduction gear ratio of $3: 1$ and a wheel radius of $0.33 \mathrm{~m}, 500 \mathrm{rpm}$ equals $20 \mathrm{~km} / \mathrm{h}(=5.56 \mathrm{~m} / \mathrm{s})$ as the rated speed. Those values satisfy the velocity profile shown in Fig. 3.4. Acceleration profile is obtained from the velocity profile, as shown in Fig 3.5.

From equation (3-5), the desired bicycle torque $T_{w}$ and bicycle speed $w_{w}$ can be calculated as shown in Fig. 3.6.

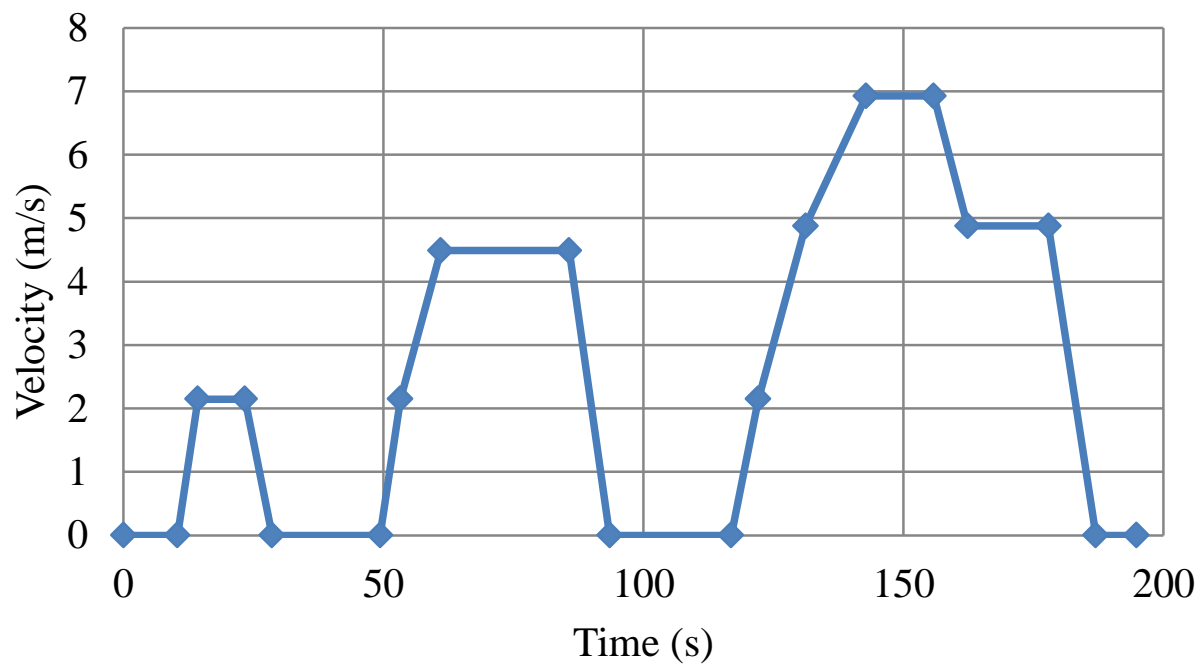

Fig. 3.4. Typical urban bike velocity cycle. 
M.A.Sc. Thesis - Jianing Lin; McMaster University - Mechanical Engineering.

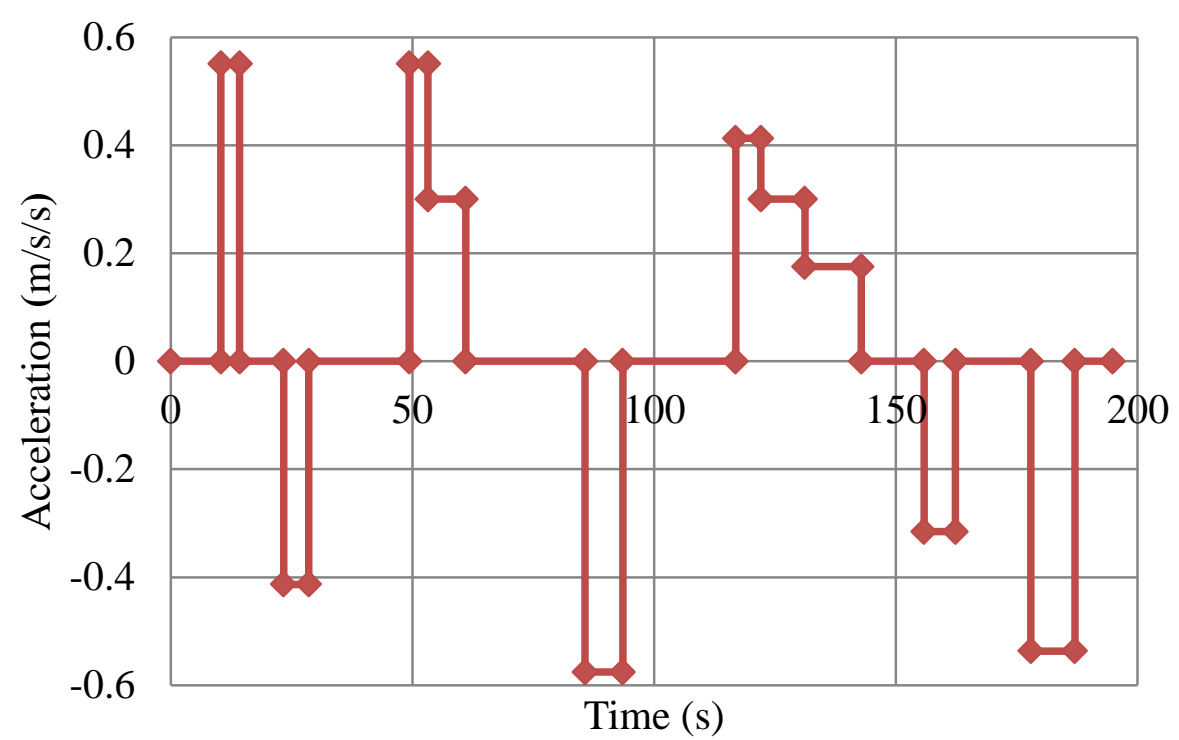

Fig. 3.5. Typical urban bike acceleration cycle.

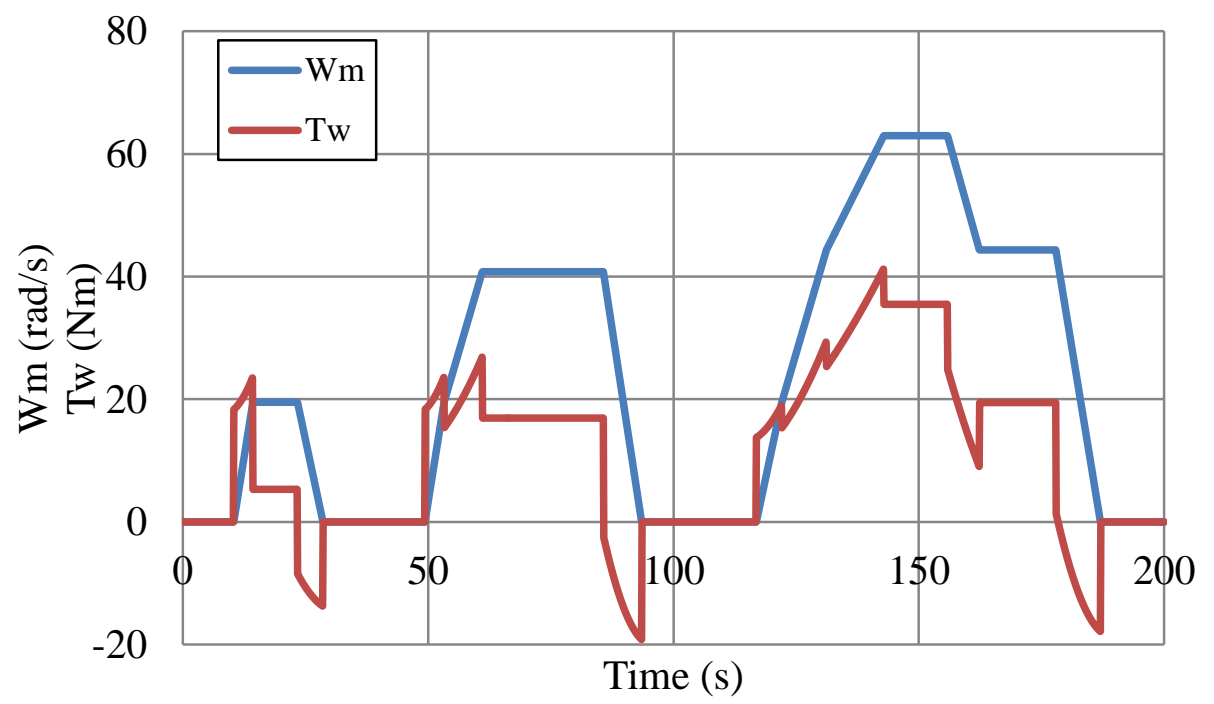

Fig. 3.6. Calculated desired bicycle torque and speed.

The desired total torque $T_{d}$, motor output torque, $T_{m}$, and human pedal torque $T_{p}$ are derived from equation (3-6) and the logic flow diagram of Fig. 3.3 is presented in Fig. 3.7. 
M.A.Sc. Thesis - Jianing Lin; McMaster University - Mechanical Engineering.

At the same time, different components of power, total desired power $P_{d}$, motor output power $P_{m}$ and human pedal power $P_{p}$, are also as shown in Fig. 3.8.

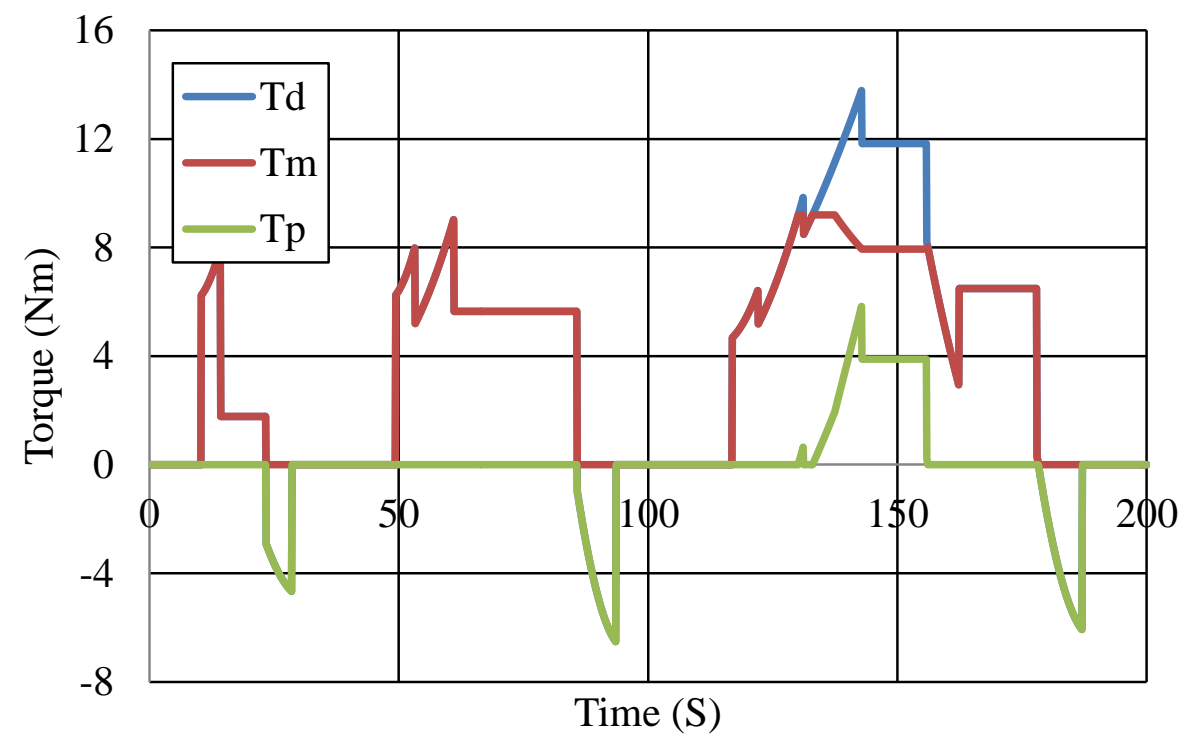

Fig. 3.7. Calculated E-Bike torque components for one velocity cycle.

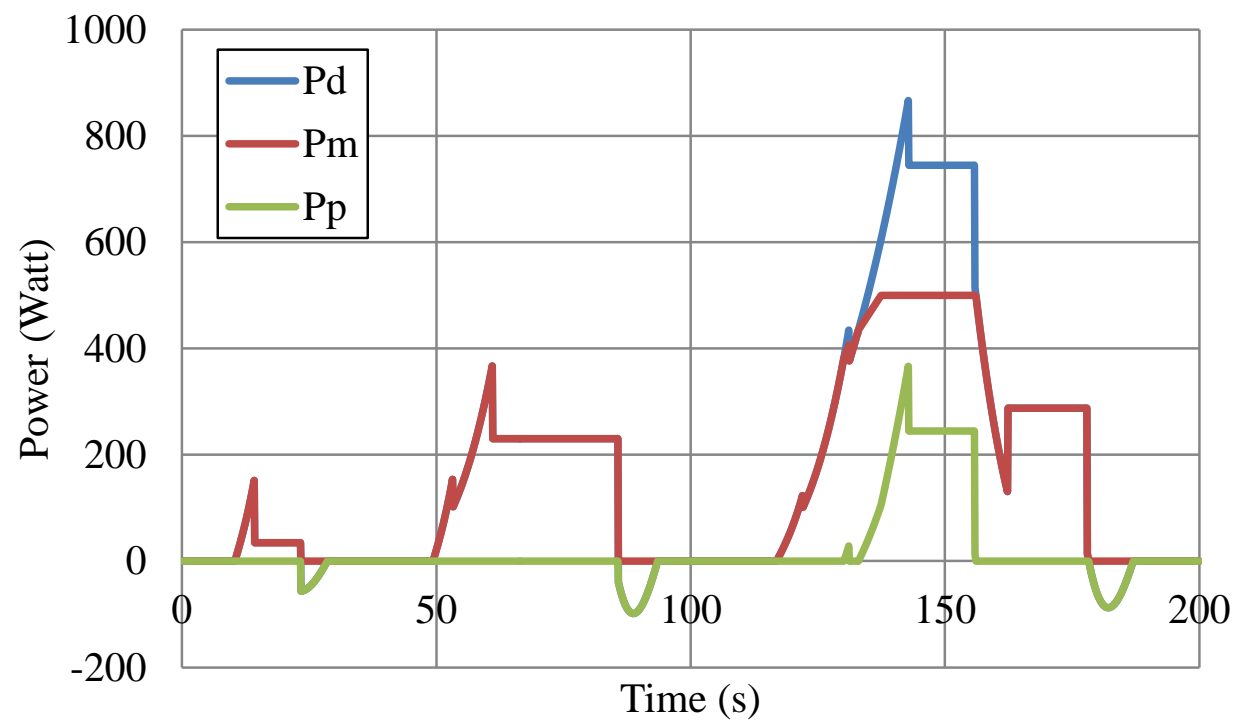

Fig. 3.8. Calculated E-Bike power components for one velocity cycle. 
M.A.Sc. Thesis - Jianing Lin; McMaster University - Mechanical Engineering.

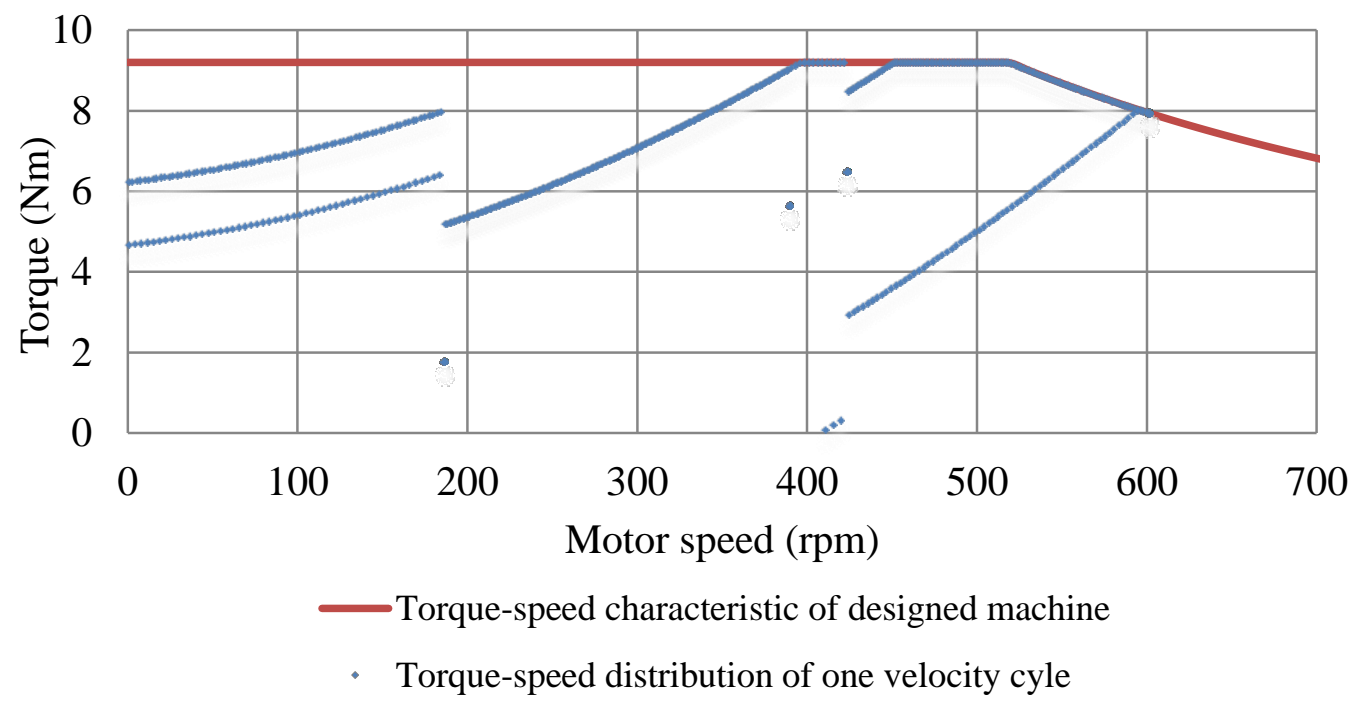

Fig. 3.9. Torque-speed envelope and operating points on the velocity cycle.

A fit individual as known can sustain $350 \mathrm{~W}$ for about 10 minutes, and up to $600 \mathrm{~W}$ for a few seconds [39]. Thus, the power profile is achievable with the cooperation between human pedal and electric machine.

All the operation points are calculated from the velocity cycle and listed in Fig. 3.9. A well-designed motor should be able to provide a torque-speed curve to totally cover all the operation points calculated from the velocity cycle, as shown below.

\subsection{Design Procedure for a SRM}

The design process starts with the requirements already obtained from the analysis above, which can be used as the design reference. The design should make sure that all of them are met. Those dimensions are summarized in Table 3.3. 
M.A.Sc. Thesis - Jianing Lin; McMaster University - Mechanical Engineering.

Table 3.3 Restrictions of the designed SRM.

\begin{tabular}{l|c}
\hline Mechanical power output & $500 \mathrm{Watt}$ \\
\hline Normal speed & $500 \mathrm{rpm}$ \\
\hline Normal torque & $9.5 \mathrm{Nm}$ \\
\hline Battery supply voltage & $48 \mathrm{~V}$ \\
\hline Peak of phase current & $35 \mathrm{~A}$ \\
\hline Rotor outer diameter & $220 \mathrm{~mm}$ \\
\hline Machine axial length & $25 \mathrm{~mm}$ \\
\hline Soft magnetic core material & M19 steel \\
\hline
\end{tabular}

Knowing the defined boundary conditions and specification requirements, the design process can be initialized. However, there are still too many unknown parameters. To avoid choosing randomly, the commercial machine design software, SPEED PC-SRD [40] is used to assist the SRM geometry analysis and design optimization. With the input of dimensions and other constraints, the SPEED PC-SRD software can be used to generate some basic information of the designed machine, for example power and torque. However, to obtain a detailed analysis, finite element analysis (FEA) is still necessary. There are a number of FEA software packages available on the market. Flux 2D from Magsoft is used in this design [41]. As the 3D model is time-consuming and over-complex, thus 2D model is used in the design synthesis. The design steps follow the logic flow diagram illustrated in Fig. 3.10. 
M.A.Sc. Thesis - Jianing Lin; McMaster University - Mechanical Engineering.

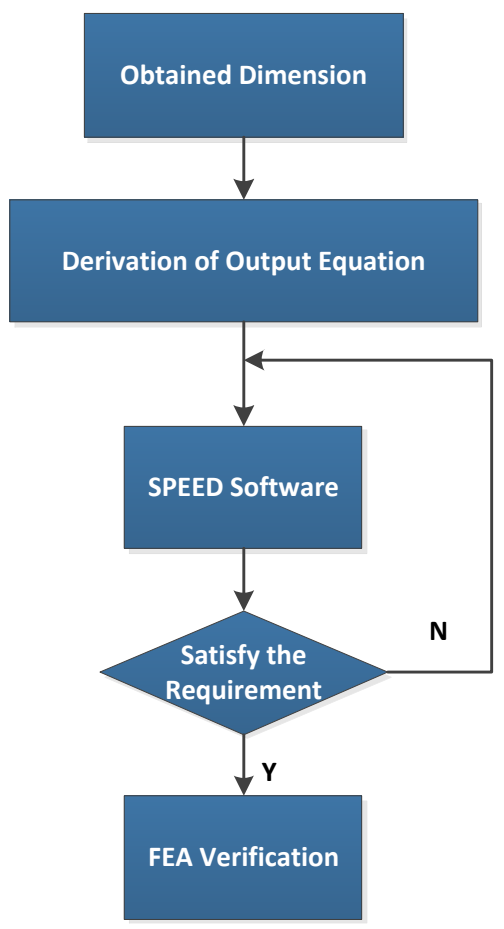

Fig. 3. 10. Design process logic flow diagram.

\subsubsection{Machine sizing and dimensions}

How to choose an optimal structure for a certain application is always an important issue for the motor design. A widely used approach is to start with the output equation. Output power can be derived from the input power as shown in equation (3-8)

$$
P_{\text {out }}=k_{e} \cdot k_{d} \cdot V_{D C} \cdot i \cdot m
$$

where $k_{e}$ and $k_{d}$ are efficiency and duty cycle ratio, $V_{D C}$ is the input voltage, $i$ is phase current, and $m$ is the phase number.

$$
V_{D C}=R_{s} \cdot i+\frac{d \lambda}{d t} \approx \frac{d \lambda}{d t}
$$


M.A.Sc. Thesis - Jianing Lin; McMaster University - Mechanical Engineering.

As for the motor operation, which can be seen as the repeat of the process from unaligned position to aligned position, $V_{D C}$ then can be expressed by equation (3-10).

$$
V_{D C} \cong \frac{d \lambda}{d t}=\frac{\lambda_{a}-\lambda_{u}}{\beta_{s} / w_{m}}
$$

Where $\lambda_{a}$ and $\lambda_{u}$ are flux-linkage at aligned and unaligned commutation positions and $\beta_{s}$ is stator pole arc. $w_{m}$ is the angular speed, which can be derived by revolutions per minute $N$.

$$
w_{m}=\frac{N \cdot 2 \pi}{60}
$$

And at aligned position, flux-linkage can be implied from equation (3-12).

$$
\lambda_{a}=\phi \cdot T_{p h}=B \cdot \frac{D}{2} \cdot \beta_{s} \cdot L \cdot T_{p h}
$$

Since saturation happens at aligned position, $B$ is the saturation flux density. $D$ is the bore diameter, $L$ is the axial length and $T_{p h}$ is the number of turns per phase. Summarizing equation (3-8) to (3-12), the output power is then expressed in equation (3-13).

$$
P_{\text {out }}=k_{e} \cdot k_{d} \cdot \frac{\pi}{60} \cdot\left(1-\frac{\lambda_{u}}{\lambda_{a}}\right) \cdot B \cdot D \cdot L \cdot T_{p h} \cdot N \cdot i \cdot m
$$

For $k_{s}=1-\lambda_{u} / \lambda_{a}$

$$
P_{\text {out }}=k_{e} \cdot k_{d} \cdot \frac{\pi}{60} \cdot k_{s} \cdot B \cdot D \cdot L \cdot T_{p h} \cdot N \cdot i \cdot m
$$


M.A.Sc. Thesis - Jianing Lin; McMaster University - Mechanical Engineering.

Equation (3-14) shows the necessity of initializing these parameters to begin the machine design.

\subsubsection{External rotor design}

The external rotor design has the advantage of lighter weight [42], which is also easier for manufacturing. That's one of the most important reasons for hub motors' popularity on electric vehicles.

During the E-bike market researching, it's found out that most of the brushed DC hub motors use direct drives; however it suffers poor torque ability, and large size [16]. As for brushless, direct driving is not a good choice. One of BLDC's most popular advantages is their larger speed range, but the E-bike is a low speed application. Thus, gear box is necessary to reduce the speed to an acceptable level, which also increases the output torque [43]. But there will introduce a mechanical energy transmission efficiency drop.

To meet the requirement of in-wheel structure of E-bike [28], and make the machine thickness small enough, a gear box located outside the rotor is chosen for this design, which is already shown in Fig. 3.1.

\subsubsection{Number of phases}

For phase number selection, the issues to be considered are the starting ability, one or two direction drive, reliability, power density, efficiency as well as the cost. As known, the conventional one-phase structure SRM has no self-starting ability and the twodirection drive requirement necessitates the phase number to be more than two. At the same time, SRM reliability and power density is improved when the phase number 
M.A.Sc. Thesis - Jianing Lin; McMaster University - Mechanical Engineering.

increases, while its torque ripple will be reduced significantly. Therefore, it seems a higher phase number is preferred.

However, the increased phases will need more power electronics switches, which increases the design cost and decrease efficiency especially at high speeds. Considering all of these factors, a traditional 3-phase SRM has been chosen because it can meet all the basic requirements with a simple structure. Most importantly, it offers a lower cost.

\subsubsection{Rotor and stator pole numbers}

For a certain SRM design, it's important to figure out the poles number at the beginning. As mentioned, the idea that the number of phases can influence torque ripple has finally been given up mainly for the reason of power electronics cost. Then, torque ripple reduction can also be achieved by increasing the number of poles.

The torque ripple frequency is determined from:

$$
f_{\text {ripple }}=\frac{w_{m}}{2 \pi} P_{r} \frac{P_{s}}{2}
$$

where $P_{r}$ and $P_{s}$ are the rotor and stator pole numbers respectively, i.e. 10 and 6 respectively in this case.

A higher rotor or stator pole number increases the torque ripple frequency proportionally. Besides, it is proved that the increased numbers of rotor poles also benefit the output average torque for SRM, although this appears at the expense of higher losses and hence lower efficiency [35]. 
M.A.Sc. Thesis - Jianing Lin; McMaster University - Mechanical Engineering.

For conventional SRMs, it's usual to have one more stator pole pair than the rotor, such as 4-2, 6-4 and 8-6. These designs have the features of good inductance ratio, high average torque, and low switching frequencies. Nevertheless, it has also been proved that a higher number of rotor poles will offer a lower torque ripple [44].

In [45] and [46], a novel SRM with 10 rotor poles and 6 stator poles was introduced. The new configuration has been proved to have a higher torque density and a lower torque ripple when compared with a conventional 6-4 SRM. The proposed 6-10 structure is particularly suitable for a 3-phase external rotor design since the external rotor provides more space for the rotor poles. Thus, the 10-pole rotor and 6-pole stator structure is chosen for the E-bike application in this paper.

\subsubsection{Rotor and Stator Pole Arcs}

The ability to start the machine at any rotor position requires a consideration of the machine pole arcs imposing the following constraints. One should avoid dead zones, which have no inductance variation for any phase, leading to a zero torque region. That is to say, the following constraints need to be satisfied, which have been discussed in [22] and [44] in detail:

$$
\left\{\begin{array}{l}
\min \left(\beta_{s}, \beta_{r}\right) \geq \frac{4 \pi}{P_{r} P_{s}} \\
\beta_{s}+\beta_{r}<\frac{2 \pi}{P_{r}}
\end{array}\right.
$$

where $\beta_{s}$ is stator pole arc and $\beta_{r}$ is rotor pole arc. For the 3-phase, 6-10 external-rotor 
M.A.Sc. Thesis - Jianing Lin; McMaster University - Mechanical Engineering.

motor, this equates to:

$$
\min \left(\beta_{s}, \beta_{r}\right) \geq 12^{\circ}, \beta_{s}+\beta_{r}<36^{\circ}
$$

and $\beta_{s}<\beta_{r}$ is preferred for this design because a smaller $\beta_{s}$ provides a larger slot for the stator winding. This is particularly important for this external rotor machine. Moreover, a lower current density is suggested, which implies larger conductor crosssection, therefore requiring a smaller stator pole arc, since current density has significant influence on the copper loss. Increasing the copper loss will not only reduce the motor efficiency, but might also cause serious thermal problems. This is especially important for this external rotor machine where winding cooling is more difficult.

A comparison between two stator pole arcs, $\beta_{s}=15^{\circ}$ and $\beta_{s}=17^{\circ}$, both with $\beta_{r}=16^{\circ}$, is made in Table 3.3.

Table 3.4 Loss comparison for different stator pole arcs.

\begin{tabular}{l|l|l|l|l}
\hline \multirow{2}{*}{$\beta_{s}$} & \multicolumn{2}{|c|}{ AWG 11 } & \multicolumn{2}{c}{ AWG 9 } \\
\cline { 2 - 5 } & $\begin{array}{c}\text { Fill } \\
\text { factor }\end{array}$ & $\begin{array}{c}\text { Copper } \\
\text { loss (W) }\end{array}$ & $\begin{array}{c}\text { Fill } \\
\text { factor }\end{array}$ & $\begin{array}{c}\text { Copper } \\
\text { loss (W) }\end{array}$ \\
\hline $15^{\circ}$ & 0.36 & 68.6 & 0.54 & 43.0 \\
\hline $17^{\circ}$ & 0.48 & 68.6 & 0.74 & 43.0 \\
\hline
\end{tabular}

For AWG9, Table 3.3 shows that a bigger stator pole arc increases the slot filling factor. As required, the filling factor is practically better to be less than 0.6 (total copperto-slot area) to reduce manufacturing difficulty. Therefore, if a $\beta_{s}=17^{\circ}$ is chosen, the 
M.A.Sc. Thesis - Jianing Lin; McMaster University - Mechanical Engineering.

conductor cross-section area is decreased leading to a coil wire section of AWG 11. But this will result in a resistance increase, leading to a higher copper loss. Thus, $\beta_{s}<\beta_{r}$ is chosen for this design.

In addition, it has been proved that the rotor pole arc has a limited influence on the torque performance. It only results in width variation of the torque impulse. However, the magnitude is practically the same, as shown in Fig. 3.11. The torque profiles for $\beta_{r}=16^{\circ}$ and $\beta_{r}=18^{\circ}$ are studied here with a constant stator pole value $\beta_{s}=15^{\circ}$. It is shown that their maximum torque is almost the same and is about $8.5 \mathrm{Nm}$. The only the difference is that the torque of $\beta_{r}=18^{\circ}$ starts to increase $0.5^{\circ}$ earlier than $\beta_{r}=16^{\circ}$, which is much smaller compared with the $2^{\circ}$ rotor pole arc increase. Therefore, a much bigger rotor pole arc is not necessary, but a slightly bigger $\beta_{r}$ is preferred.

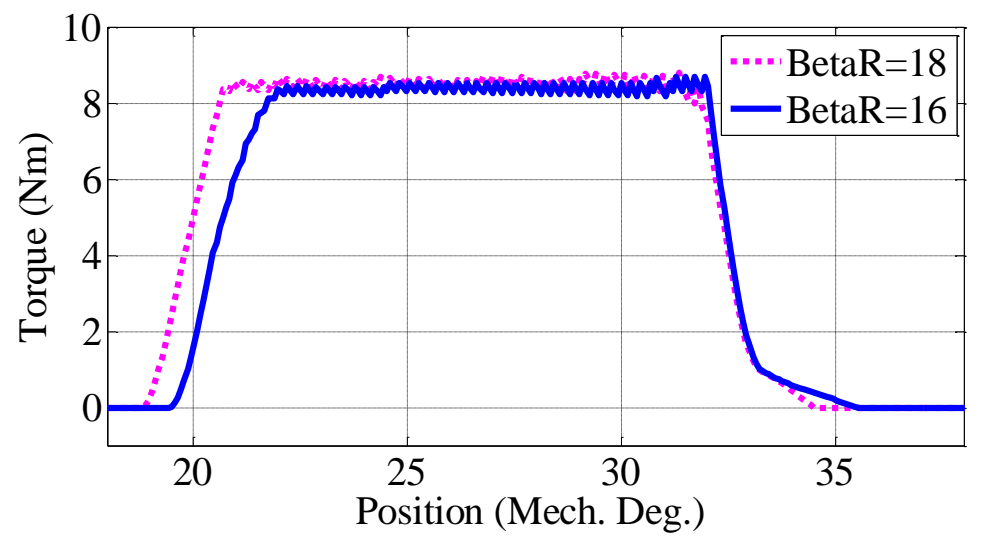

Fig. 3.11. Torque performance comparison for different rotor pole arcs.

\subsubsection{Winding Design}

The phase winding in a SRM is featured of having all turns of wires around one tooth, 
M.A.Sc. Thesis - Jianing Lin; McMaster University - Mechanical Engineering.

resulting in phases electrically isolated with each other. This is called concentrated winding, leading to higher fault tolerance than conventional motors, especially AC machines.

Fig. 3.12 shows the structures of the conventional concentrated winding. As known, the concentrated winding has the advantages of shorter and less complex end-windings, which is significantly important for this short length SRM design. Because end winding analysis is one of the most complex issues for the machine design study, which will bring the problem that the analyzed motor can't represent the real designed prototype machine, it will definitely increase the design difficulty. At the same time, the concentrated winding also has the advantage of lower copper losses and higher efficiency. However, it also brings the problem of bigger torque ripples than distributed winding. More attention is needed to reduce the torque ripples.

Apart from the winding method, another important issue is the winding filling factor. Due to spatial distribution of coils and the winding manufactory technologies, not all $100 \%$ of space can be used. And filling factor is sometimes the most important matter that limits the designed motor's performance, like the power over volume ratio. In this paper, the filling factor is defined as the ratio of effective copper wire area over the slot area. With some specific technology, the winding filling factor can be made even 0.8, and typically within 0.6 , however, much lower for hand-winding. For the prototype motor in this thesis, winding is going to be made by hand. Thus, it's better to be made within 0.4 , whose value is set by practical experience. 
M.A.Sc. Thesis - Jianing Lin; McMaster University - Mechanical Engineering.

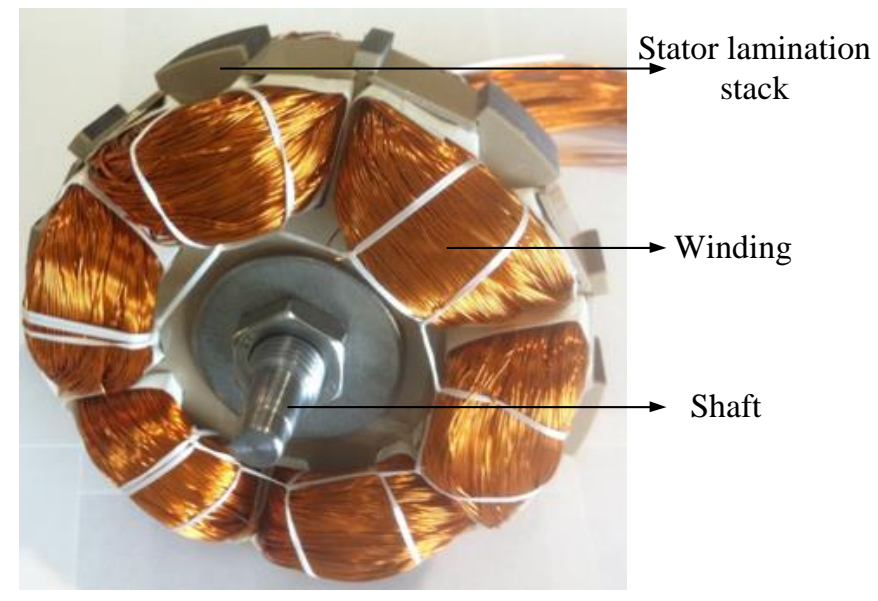

Fig. 3.12. Physical view of SRM concentrated winding.

Considering the winding sequences, there are two options. One is the traditional winding, called NNN-SSS in this paper, as shown in Fig. 3.13 (a). But another winding sequence, called NSN-SNS is tried in this paper as shown in Fig. 3.13 (b). The reason for choosing NSN-SNS is that, as shown in Fig. 3.13 (a), when the stator follows an energizing sequence of A-B-C, the space vector of magnetic field changes -30 degree for the commutation from energized phase $\mathrm{A}$ to phase $\mathrm{B}$, and another -30 degree for phase $\mathrm{B}$ to phase $\mathrm{C}$, however, it turns to be 120 degree when phase $\mathrm{C}$ is turned off, but phase $\mathrm{A}$ is turned on for another repeat of the energizing phase sequence. This will introduce imbalance between $\mathrm{C}$ to $\mathrm{A}$ phase changing and the other two phase changing commutation points. The NSNSNS is suggested to avoid this imbalance. As seen in Fig. 3.13(b), with the same A-B-C energizing sequence, they are all 120 degree vector angle changing for all A-B, B-C and C-A phase changing commutation points. 
M.A.Sc. Thesis - Jianing Lin; McMaster University - Mechanical Engineering.

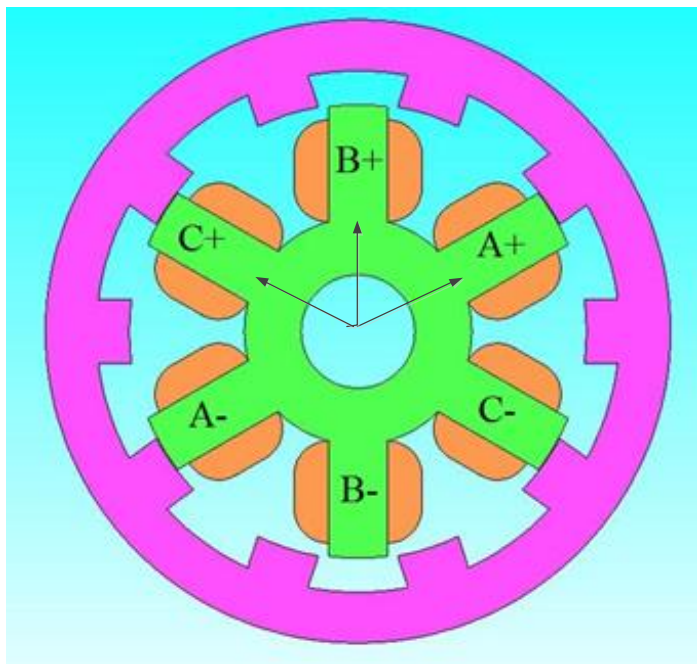

(a) NNNSSS

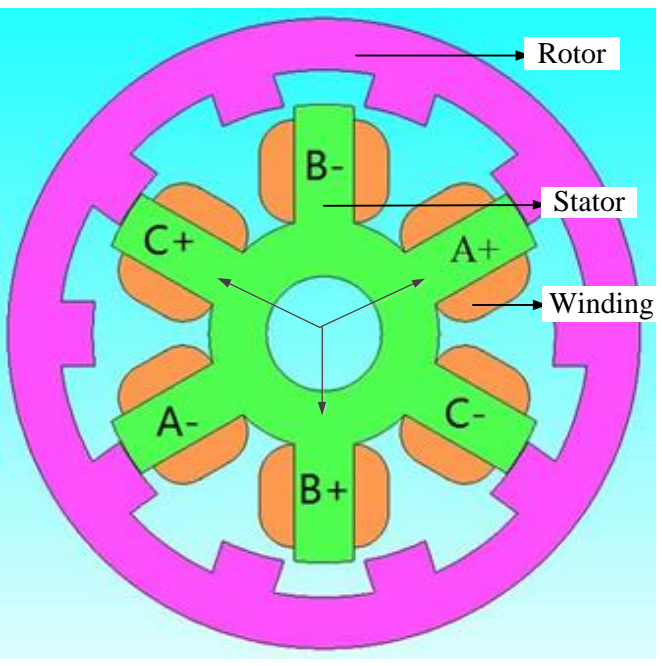

(b) NSNSNS

Fig. 3.13. Winding and energizing sequence comparison.

Comparison of these two winding sequences will be conducted in FEA, which will be shown in the FEA verification.

\subsection{Design solutions}

Considering the weight, output power, efficiency, and torque performance as well as thermal influence, the final design is shown in Fig. 3.14, with relevant parameters in Table 3.5. Motor performance is presented in Table 3.6. 
M.A.Sc. Thesis - Jianing Lin; McMaster University - Mechanical Engineering.

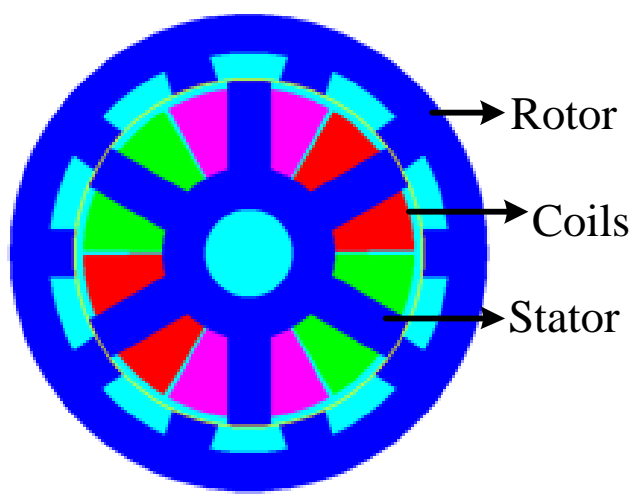

Fig. 3. 14. Designed SRM.

Table 3.5 Structure parameters.

\begin{tabular}{l|l}
\hline Number of stator poles & 6 \\
\hline Number of rotor poles & 10 \\
\hline Rotor outer diameter & $220 \mathrm{~mm}$ \\
\hline Stator bore diameter & $40 \mathrm{~mm}$ \\
\hline Air gap & $0.4 \mathrm{~mm}$ \\
\hline Length & $25 \mathrm{~mm}$ \\
\hline Stator pole arc & $15^{\circ}$ \\
\hline Rotor pole arc & $16^{\circ}$ \\
\hline Turns per phase & 104 \\
\hline Wire size & AWG \#8 \\
\hline Core material & $\mathrm{M} 19$ steel \\
\hline
\end{tabular}

Table 3.6 Performance of the 6-10 external rotor SRM.

\begin{tabular}{l|l}
\hline Rated power & $504.4 \mathrm{~W}$ \\
\hline Rated speed & $500 \mathrm{rpm}$ \\
\hline Torque & $9.6 \mathrm{Nm}$ \\
\hline Voltage & $48 \mathrm{~V}$ \\
\hline Current & $35 \mathrm{~A}$ \\
\hline Mass & $7.1 \mathrm{~kg}$ \\
\hline
\end{tabular}


M.A.Sc. Thesis - Jianing Lin; McMaster University - Mechanical Engineering.

\subsection{Design verification with FEA}

As discussed, to obtain detailed information, like the flux-linkage, torque and inductance at different rotor positions with different currents, which are most important for a designed machine and also of necessary to figure out its control strategies, FEA is conducted. However, 3D model is time-consuming and of lower efficiency. 2D modal is used. But to take this step, there is necessity to check the accuracy of 2D FEA analysis and also verify the result from the calculation above.

\subsubsection{D FEA accuracy verification}

It's first compared the results from both SPEED and 2D model in FEA. As known, the flux-linkage profiles of unaligned and aligned positions are definitely important for the motor performance. They determine not only the output torque but also the output power. Thus, the flux-linkage results from these two software are compared in Fig 3.15.

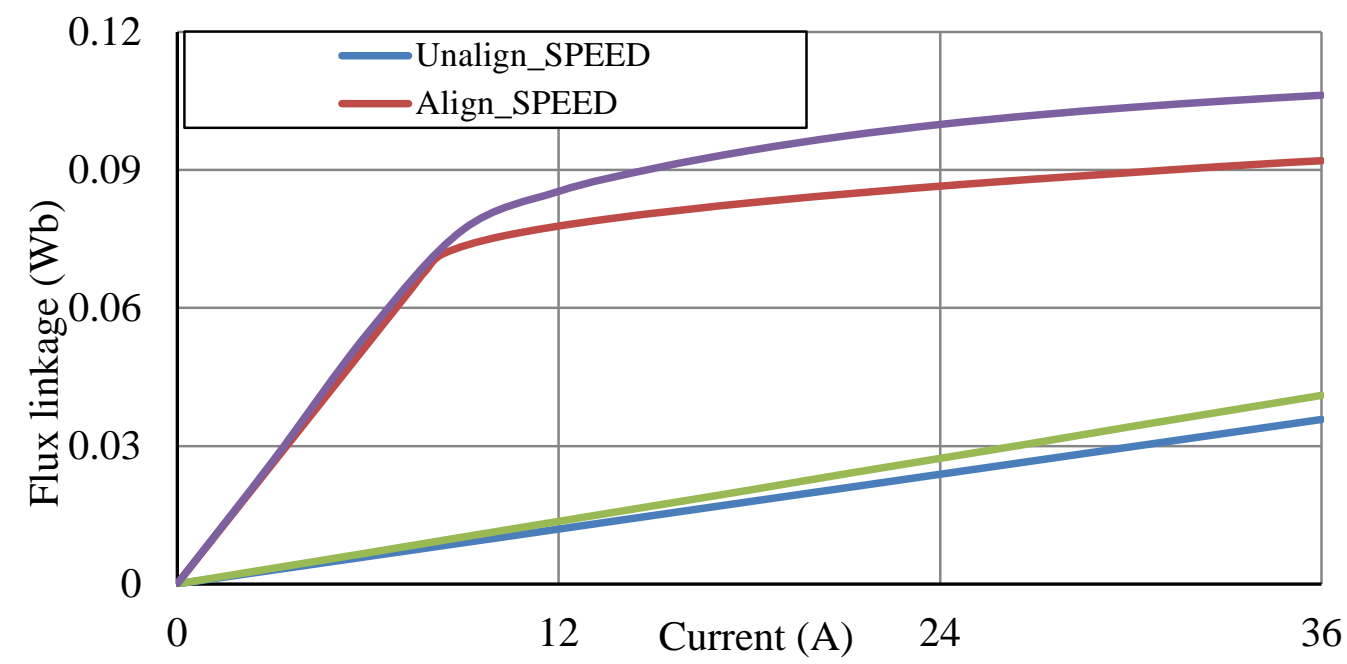

Fig. 3. 15. Flux linkage versus current comparison at aligned and unaligned rotor commutation positions. 
M.A.Sc. Thesis - Jianing Lin; McMaster University - Mechanical Engineering.

From it, it's seen that for unaligned potions, the difference is smaller and slopes of the two lines are a little different. As for the aligned position, the difference for unsaturated current is quite smaller, but it turns to be bigger after the saturated point. And the biggest difference appears at the maximal current of $36 \mathrm{~A}$, which is about $10 \%$. At the same time, the saturated points are also different for SPEED and FEA. There are some reasons for this. One is caused by the material data for M19 steel in these two software are different with each other. Another reason is the calculation method difference. To determine which is more accurate, comparison with the experiment will be conducted in last chapter.

At the same time, the different inductance values are listed in Table 3.7, with $\mathrm{Lu}$ as the inductance at unaligned position, Lau as the inductance at aligned position before saturation. Ls is also at aligned position but after saturation, while Las is defined as fluxlinkage over current. In Table 3. 7, the differences are all acceptable. Thus, the FEA is used to obtain more information.

Table 3.7 Inductance comparison between FEA and SPEED.

\begin{tabular}{c|l|l|l}
\hline & FEA $(\mathrm{mH})$ & SPEED $(\mathrm{mH})$ & Difference $(\%)$ \\
\hline $\mathrm{Lu}$ & 1.1 & 1.0 & 9.09 \\
\hline $\mathrm{Lau}$ & 8.5 & 8.7 & 2.35 \\
\hline $\mathrm{Ls}$ & 0.408 & 0.400 & 2.44 \\
\hline Las & 2.952 & 2.956 & 0.14 \\
\hline
\end{tabular}

To verify that the $2 \mathrm{D}$ model is enough for this design, a comparison between a $3 \mathrm{D}$ model in JMAG and 2D model in Flux 2D is studied in Fig. 3.16. The result is the total torque with the same turn-on and turn-off angle control and both are one-phase-energizing 
M.A.Sc. Thesis - Jianing Lin; McMaster University - Mechanical Engineering.

at one time. As no enough information for 3D model, the difference are very small. Finally, only 2D model is used in FEA to save time.

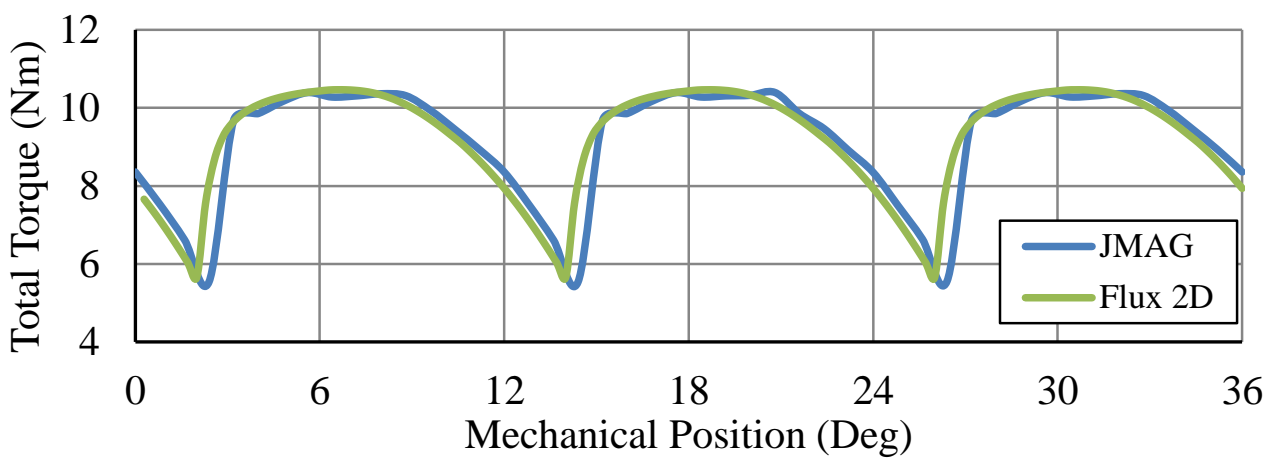

Fig. 3. 16. Total torque comparison between 2D and 3D models.

\subsubsection{Results from 2D FEA}

Flux-linkage distributions at unaligned and aligned commutation positions are shown in Fig. 3.17. The motor flux-linkage and torque versus mechanical rotor positions with different current, from 2D FEA are shown in Fig. 3.18 and Fig 3.19.

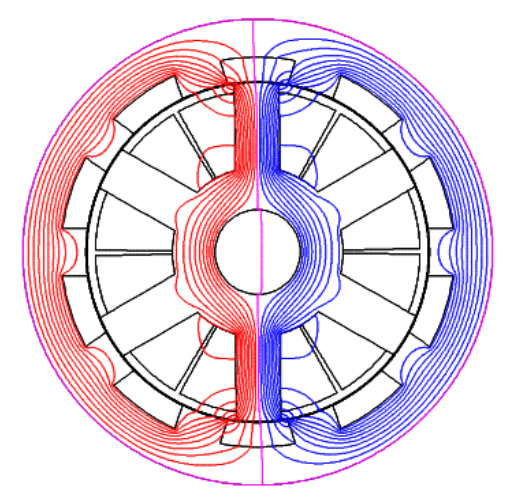

Unaligned commutation position

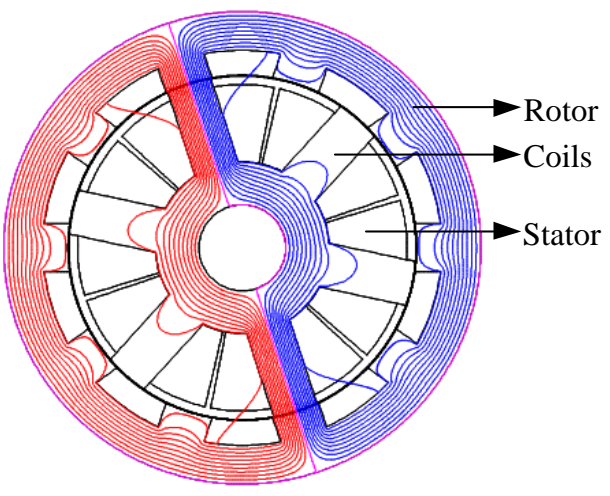

Aligned commutation position

Fig. 3.17. Flux path for unaligned and aligned commutation positions. 
M.A.Sc. Thesis - Jianing Lin; McMaster University - Mechanical Engineering.

As already shown in Fig. 3.15, the saturation starts (for the aligned position), when the current increases to about 9A. This can also be explained in Fig. 3.18. Aligned position is of 18 mechanical degree in this thesis. As seen, when the current is less than 9A, the flux linkage increases proportionally with the current, and the increasing is significantly large, which shows a big space between the curves for a different current. However, the increase with currents turns to be very slow after 9A. That means that a much smaller inductance and saturation happens.

Saturation is the reason for SRM's advantage of high torque and power density. However, it also results in a nonlinear torque-current-position characteristic of the machine. This nonlinearity introduces difficulties for SRM modeling and control. However, compared with other electric machine, SRM has the big advantage of smaller mutual inductance, which increases its faults tolerance and decreases the study difficulties. The self-inductance and mutual-inductance are compared in Fig. 3.20. It has been proved for this SRM that its mutual-inductance is less than $1 \%$ of self-inductance. Thus, mutual inductance is ignored during the analysis.

The torque profiles of the three phases are shown in Fig. 3.21. The phase difference between each is 12 degree and repeat cycle is 36 mechanical-degrees. As already shown in Fig. 3.16, with the simplest current turn-on and turn-off angles control, the total torque can be generated. And the average torque of 2D FEA in Fig. 3.16 is about 9.2N.m, a little smaller than the result from SPEED of 9.6N.m. However, the torque ripple is very big, about $52 \%$, which is using ideal current without lagging after switches turning on and off. 
M.A.Sc. Thesis - Jianing Lin; McMaster University - Mechanical Engineering.

More control work needs to be done to get this machine prepared for the E-bike application.
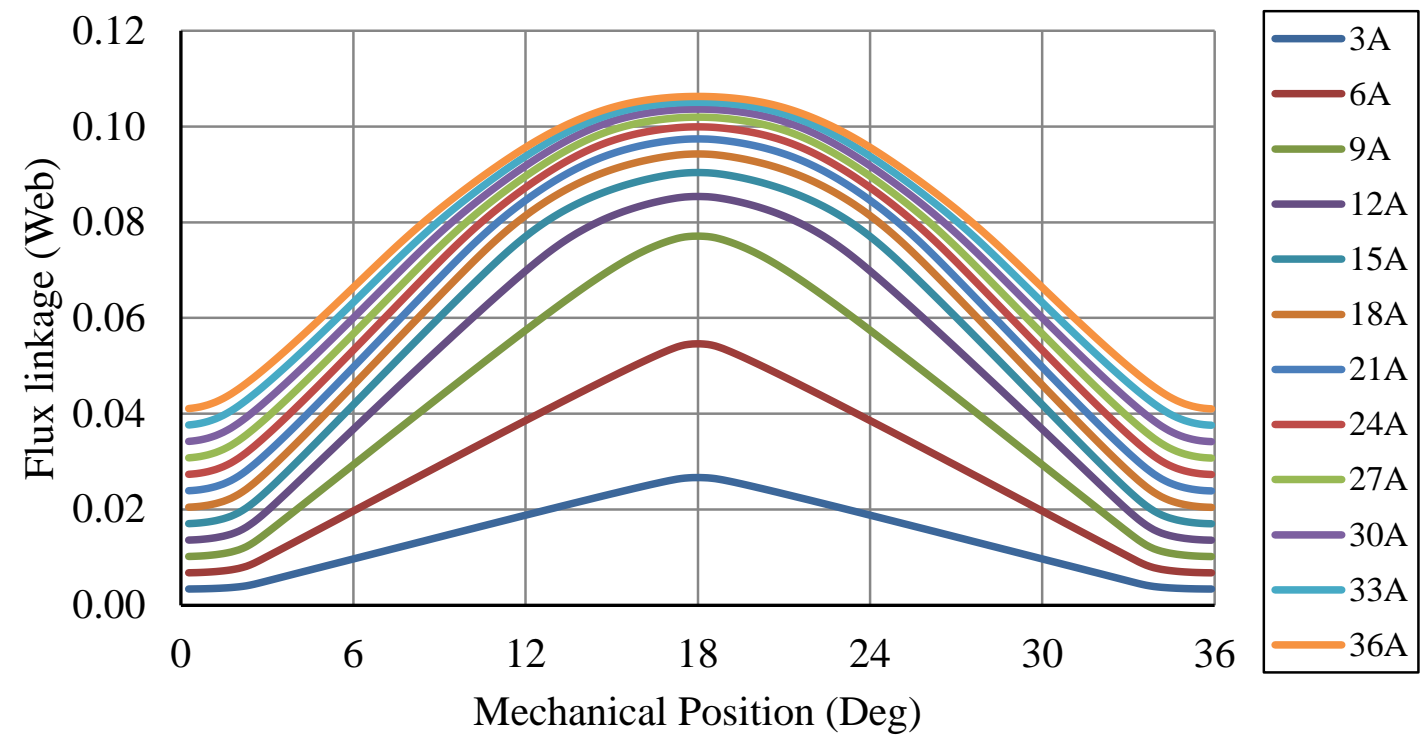

Fig. 3.18. Flux linkage versus position for different currents.
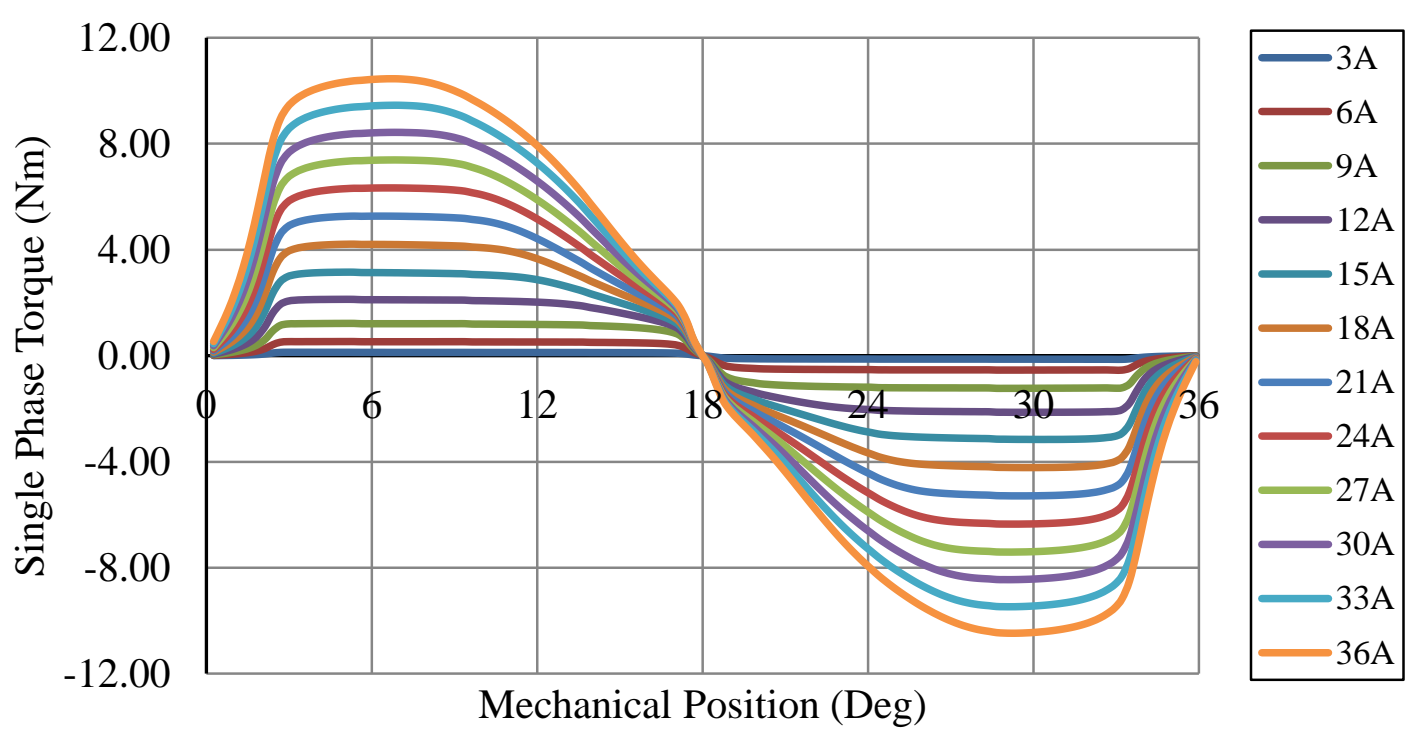

Fig. 3.19. Single phase torque versus position for different currents. 
M.A.Sc. Thesis - Jianing Lin; McMaster University - Mechanical Engineering.
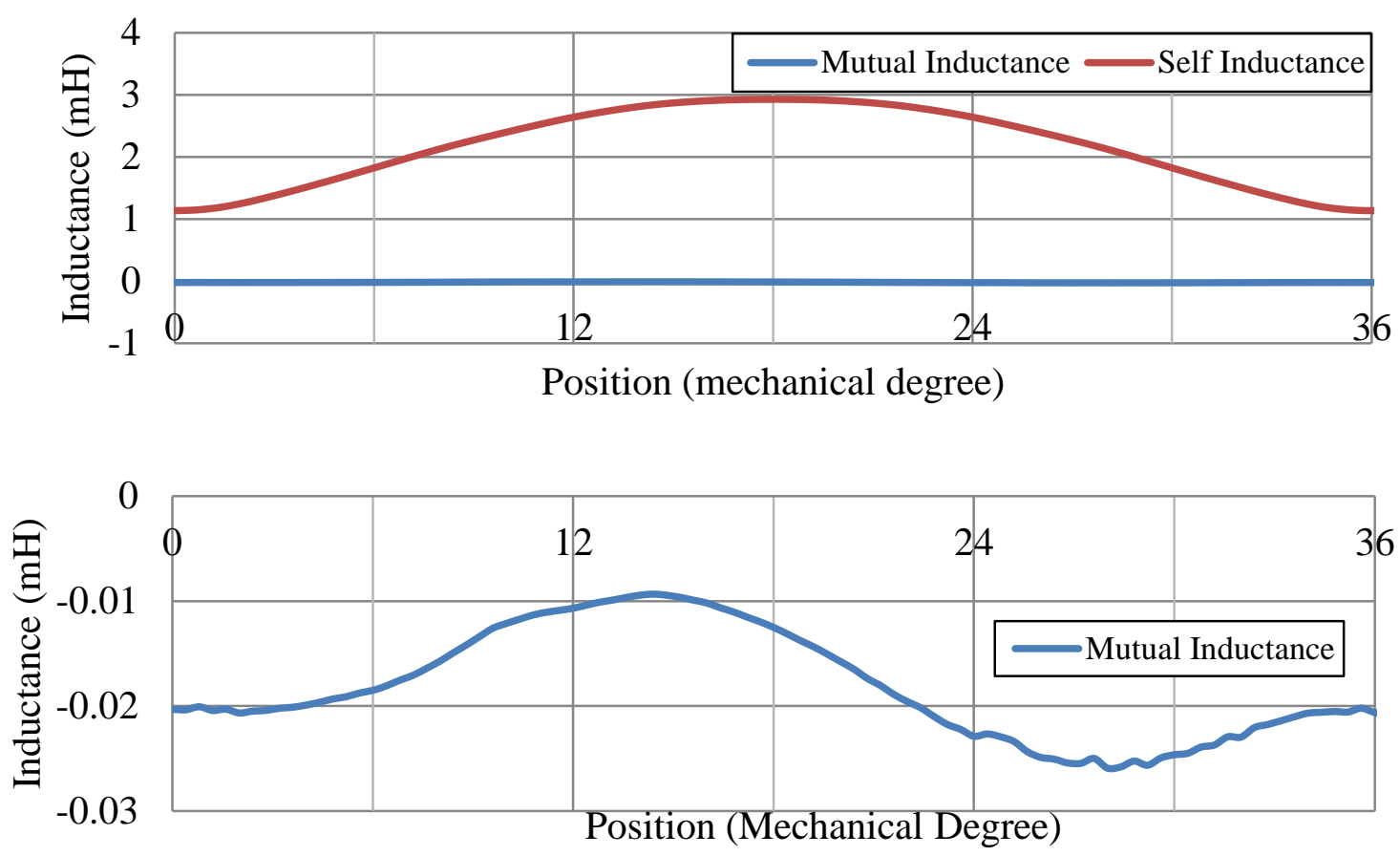

Fig. 3.20. Inductance comparison.

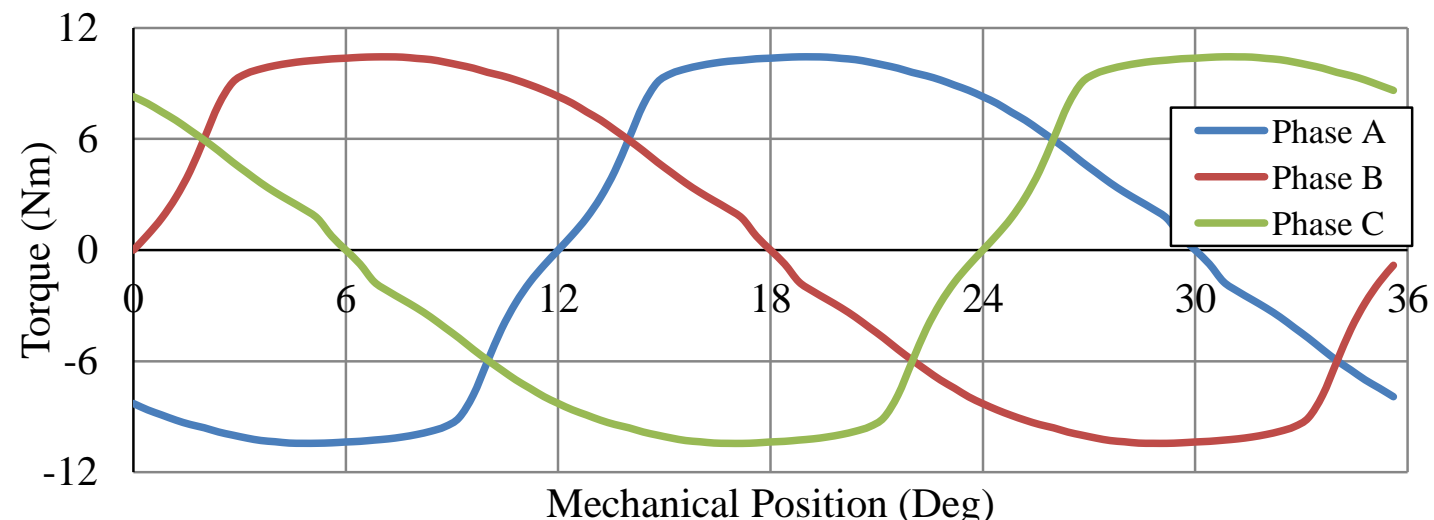

Fig. 3.21. Torque profiles for three phases.

\subsubsection{Energizing sequence}

As mentioned, for the winding and energizing sequence, NSNSNSN is preferred than NNNSSS. This is verified by checking the torque difference between these two in 2D- 
M.A.Sc. Thesis - Jianing Lin; McMaster University - Mechanical Engineering.

FEA, with the current exciting sequence shown in Fig. 3.22, which is in the turn of A-BC-A. Each phase is energized for 18 mechanical degrees, for the position range with positive torque generation. So there are overlaps between phases, like phase A and phase $\mathrm{C}$ is overlapped from 0 to 6 degree, phase A and phase B are overlapped from 12 to 18 degree. And the output torque with those current is shown in Fig. 3.23.

In Fig. 3.23 with NSNSNS winding and energizing sequence, for every 36 degree, there is a repeat for every 12 mechanical degrees. Like from 0 degree to 12 degree, the output torque is bigger from 0 to 6 degree, as there are two phases energized, however, only phase A is on from 6 to 12 degree. But for NNNSSS, there is a difference between the first and the last two. For example, the torque at 6 degree is bigger than 18 degree and 30 degree. Like for 6 degree, phase $\mathrm{A}$ is on and phase $\mathrm{C}$ is going to turn off. So there are two phase, $\mathrm{A}$ and $\mathrm{C}$ is on for this commutation points. The flux linkage distribution is studied in Fig. 3.24.

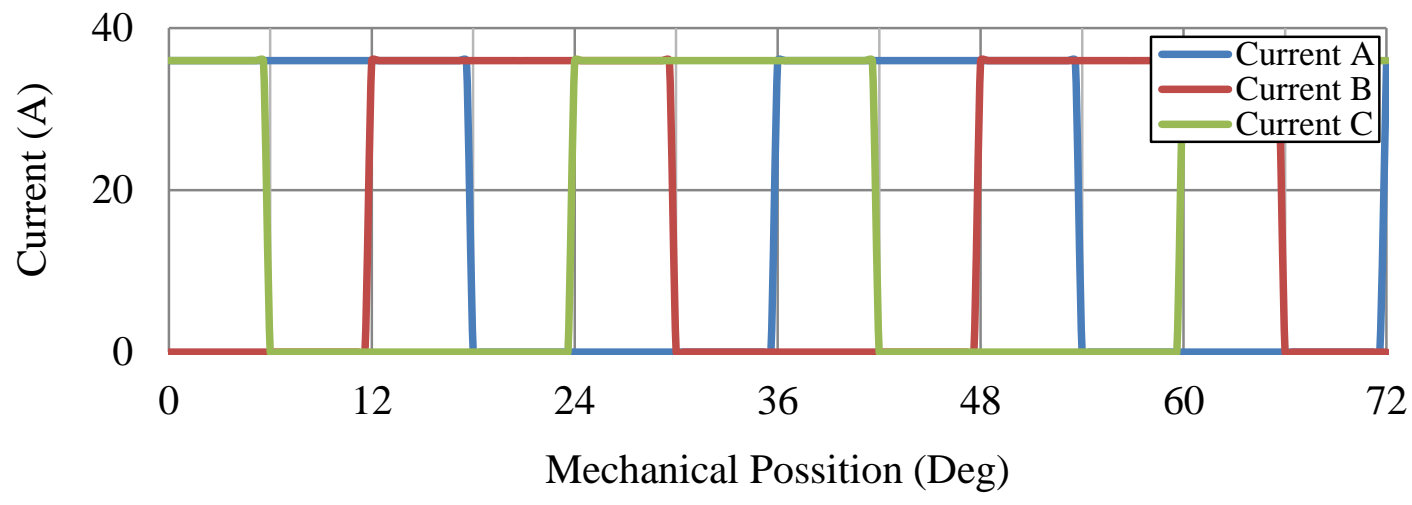

Fig. 3.22. Currents energizing sequence for the three phases. 
M.A.Sc. Thesis - Jianing Lin; McMaster University - Mechanical Engineering.

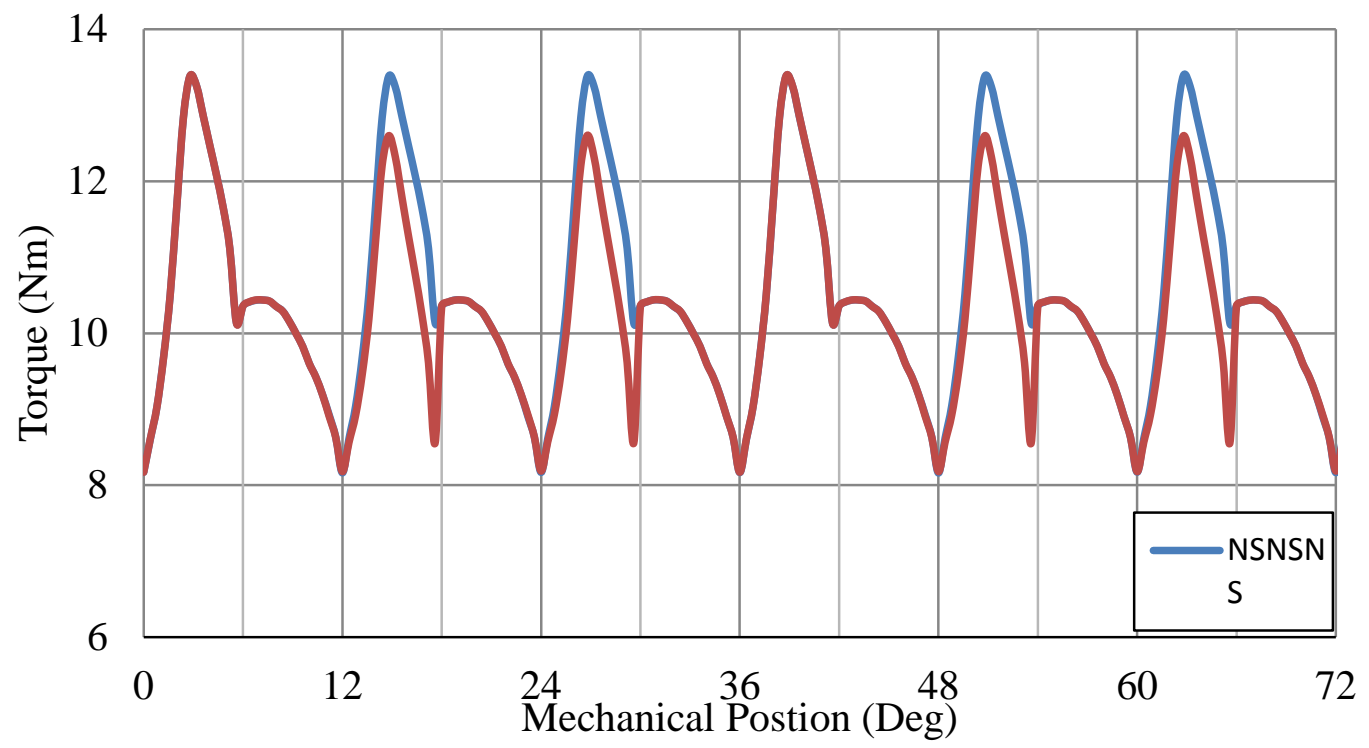

Fig. 3.23. Total torque comparison for NSNSNS and NNNSSS.
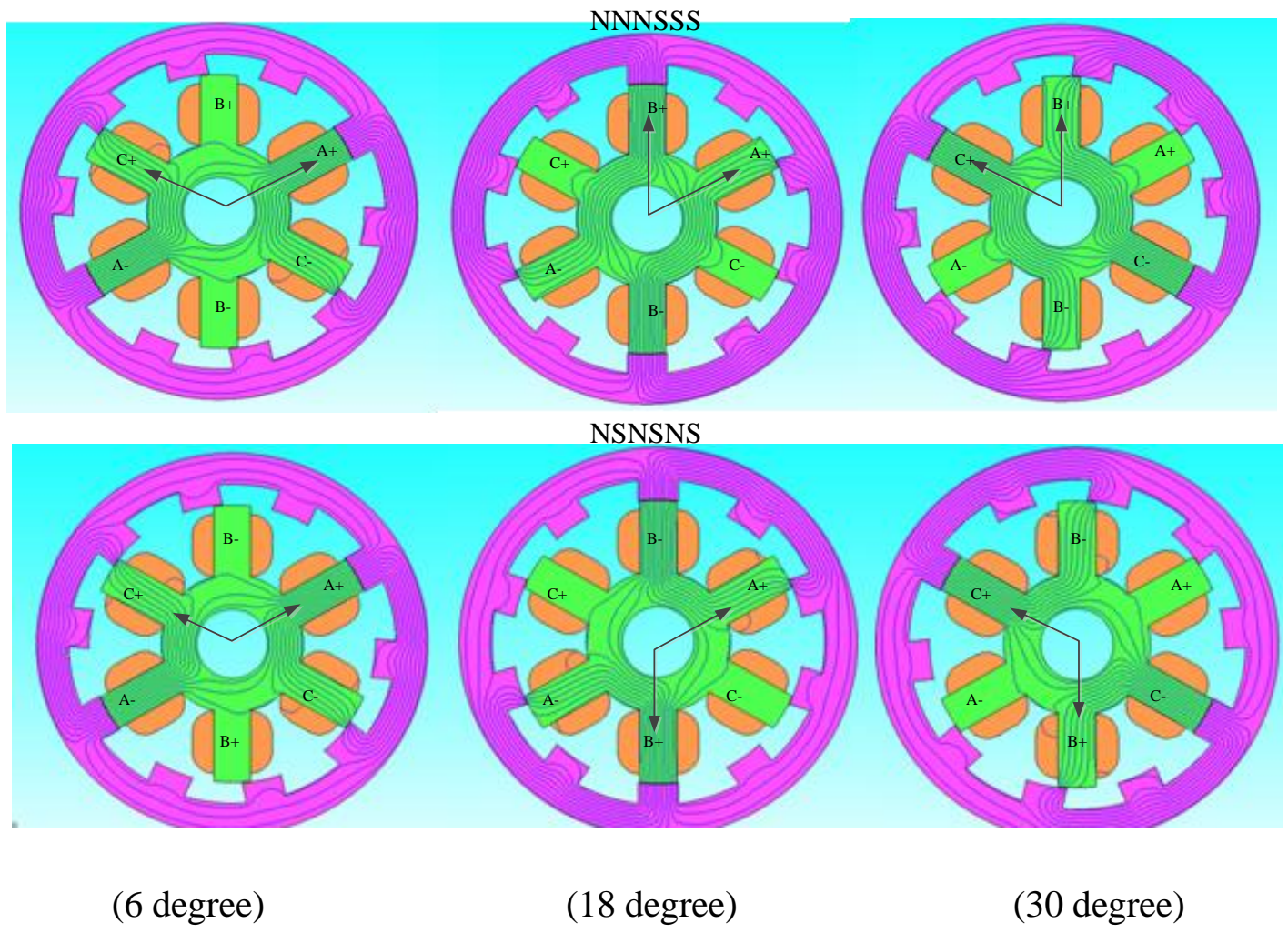

Fig. 3.24. Flux linkage distribution for NSNSNS and NNNSSS. 
M.A.Sc. Thesis - Jianing Lin; McMaster University - Mechanical Engineering.

As seen in Fig. 3.24, if the phase difference between the overlapped two phases is 30 degree, less than 90 degree, there is a strengthening effect on flux for each phase. And this will increase the saturation. The total torque of these two phase will be decreased. But for those two phases, which have a phase difference of 120 degree, they are cancelling each other out. This will decrease the saturation of each phase. Thus, for a certain phase current, it will generate more torque. And the total torque will increase. Therefore, NSNSNS winding and energizing sequence is preferred in this paper.

\subsection{Conclusion}

By studying the kinetic model and a typical velocity cycle, a 500W and $500 \mathrm{rpm}$ SRM is decided to be designed for the E-bike application in Ontario, Canada. It also requires a 3:1 reduction gear box. All the geometry diameters are discussed during the design process, which finally propose a solution of 3-phase, 6-stator-pole, 10-rotor-pole external rotor structure.

Its magnetic profile is also studied and verified by 2D FEA. Compared with conventional winding and energizing sequence, a NSNSNS is recommended, which is proved with better consistency between phases and higher torque output. 
M.A.Sc. Thesis - Jianing Lin; McMaster University - Mechanical Engineering.

\section{Chapter 4 Switched Reluctance Drive Design and Torque Ripple}

\section{Reduction}

SRM has well-known advantages of simple structure, ruggedness, high torque and power density, making it really attractive in academic area. However, its extensive development in industry has been quite slow and fluctuating. The main reason lies in its big torque ripple and noise as compared with other electric machines.

To reduce torque ripple, there are two ways. One is by optimizing SRM's geometry, like changing the phase numbers, pole numbers and different dimensions, which has been studied in Chapter 3. The other way is conducted by its drive, mainly by its specialized control strategy, which will be discussed in this chapter.

The origin of SRM torque ripple has been discussed when it is compared with other conventional electric machine. And then the SR drive construction is introduced. After that, two different control strategies, angular position control (APC) and torque sharing function (TSF) control, are compared when being applied on the designed SRM.

\subsection{Origin of Torque Ripple}

The definition of torque ripple in this thesis is shown in equation (4-1).

$$
\text { Torque ripple }=\frac{\text { Maximum torque }- \text { Minimum torque }}{\text { Average torque }} \times 100 \%
$$

Following the operation principle of minimum magnetic resistance, the salient stator pole and rotor pole always tend to get aligned, where the flux has smaller magnetic 
M.A.Sc. Thesis - Jianing Lin; McMaster University - Mechanical Engineering.

resistance but higher resistance when the two salient poles are unaligned. By commutation of different phases, the flux keep changing its loop and produce positive torques, resulting from this tendency of aligning the stator and rotor poles. This torque is called reluctance torque. Differently, SRM only has reluctance torque and no back electromotive torque. As back electromotive torque is caused by Lenz's low, thus usually, it is smoother than reluctance torque.

In addition, for traditional AC motor, it has sinusoidal stator and rotor magnetic fields. These two magnetic field work together to generate the torque. However, for SRM, its concentrated winding and double salient pole structure cannot produce sinusoidal flux distribution in circumferential direction. Together with the square wave excitation, it introduces more harmonic, which is the reason for non-smooth torque. Besides, the commutation introduced by square wave excitation will result in more torque ripples at those commutation points. As for DC motor, it also has the problem of commutation. However, DC motor is using brushes or permanent magnet, which makes multiple structure more achievable. This is one way to reduce the torque ripple.

At the same time, for the traditional AC or DC motor, they normally has two magnetic field. These two magnetic field can be controlled for most conditions, which makes the torque ripple reduction easier. As only one magnetic field definitely reduces its torque/current generation ratio to obtain higher torque, SRM has to be energized with bigger current. This will introduce deep saturation condition especially at aligned positions. The serious nonlinear characteristics of SRM also presents higher torque ripple and also makes its control strategy harder to achieve. 
M.A.Sc. Thesis - Jianing Lin; McMaster University - Mechanical Engineering.

Another reason that make SRM's torque ripple harder to be reduced is that the torque produce of SRM deeply depends on its position, not only the current. Because of the nonlinear feature, this function is also nonlinear, which is complex for not only SRM's modeling, but also its control.

\subsection{Classic Control Diagram with Angular Position Control}

There are usually three close loops for the classic control diagram. A typical control diagram is shown in Fig. 4.1.

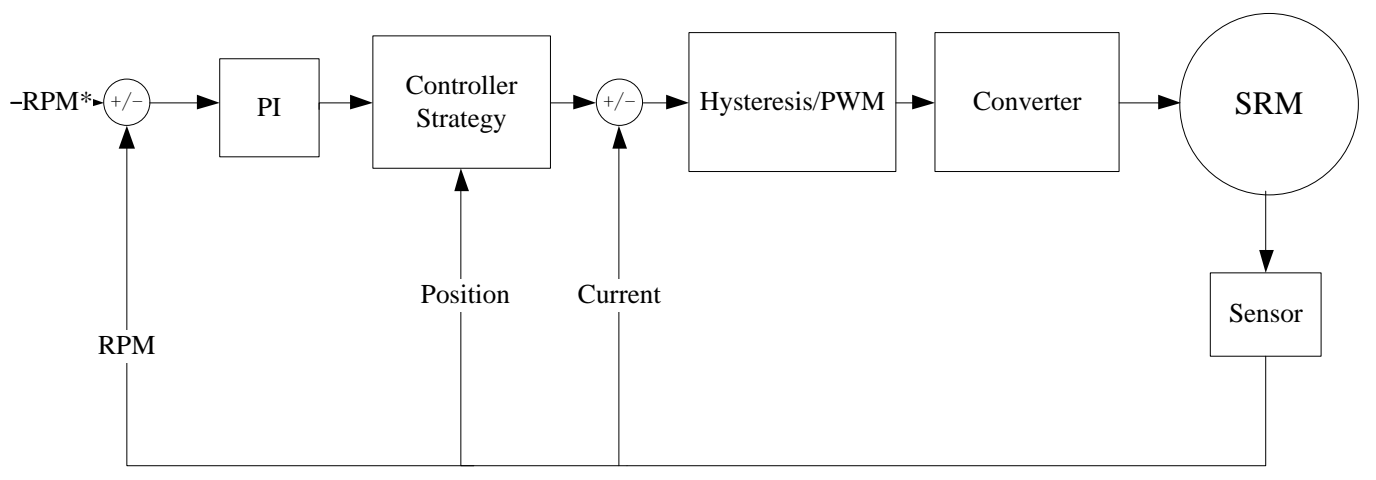

Fig. 4.1. Classic control diagram for SRM.

As seen, one loop is for the speed control, which is called speed loop. Usually, PI controller is enough for SRM's speed control. The difference between the real speed and the desired speed is the input for a PI controller, which generates a desired torque to overcome the speed difference. The reason for choosing a PI controller instead of a PID controller is to minimize the tuning complexity. At the same time, PI controller is more widely used on motor as it can keep a good track on the required speed, however, the D parameter sometimes will increase the noise of the system. Based on the torque ripple reduction consideration of this thesis, PI controller is more suitable. 
M.A.Sc. Thesis - Jianing Lin; McMaster University - Mechanical Engineering.

Another loop is to generate the signals for switches of the converter. Normally, there are hysteresis current and PWM voltage controls. In this thesis, hysteresis current control is used instead of PWM due to hysteresis control's easy implementation and fast dynamic response. But hysteresis control suffers bigger current noise, resulted from varying the switching frequency to maintain constant hysteresis current band.

The other control loop is to find out what the proper current for each phase to generate the desired torque. As discussed in chapter 2, for one phase, there is a certain range of positions, which can generate positive output torque. Controlling the phase to be energized at those positions will avoid negative torque. And to reduce the torque ripple, it also requires specific turn-on and turn-off angle, and different current magnitude at different positions. In classic control diagram, angular position control is used. Certain turn-on and turn off position is chose for each phase.

As discussed above, the single-phase energizing each time is a very simple and easy, however, it also suffers bigger torque ripple as there is no overlap between phases. The improvement applies multi-phase energizing angular control. The biggest problem for multi-phase angular control is the mutual inductance. As known, SRM is famous for its smaller mutual inductance. So each phase is independent with other. This is also proved in chapter 2. 
M.A.Sc. Thesis - Jianing Lin; McMaster University - Mechanical Engineering.

\subsection{Torque Sharing Function Control}

The idea of the torque sharing function is that multiple phases will be used to generate the total desired torque following a sharing function. If $T_{d}$ is the desired total torque, the sharing function $f(\theta)_{i}$ for the phase $i$ should meet the following constraints:

$$
\begin{gathered}
T_{i}=T_{d} \cdot f(\theta)_{i} \\
\sum_{i=1}^{m} f(\theta)_{i}=1 \\
0 \leq f(\theta)_{i} \leq 1
\end{gathered}
$$

where $T_{i}$ is the desired torque for phase $i$.

It is shown that, there is more than one unique solution for the equations of (4-2) to (44). Actually many functions of $f(\theta)_{i}$ can satisfy the constraints. However, there are usually a few factors to consider when choosing a TS function.

One is the copper losses. The torque sharing function may assign one phase to generate torque at a position of small rate of change of phase inductance. The required current will be big, but the copper losses will increase significantly. Therefore, one of the optimizing objectives to decide the proper torque sharing function is to reduce the copper losses.

Another is to maximize the speed range of a certain TS function. As known from equation (3-9) in chapter 3, voltage of the DC link $\left(V_{D C}\right)$ is constant, approximately equaling the rate-of-change of flux linkage, when ignoring the voltage drops on switching components and phase resistance. It can derive equation (4-5).

$$
\frac{d \psi_{k}}{d \theta} \approx \frac{V_{D C}}{w}
$$


M.A.Sc. Thesis - Jianing Lin; McMaster University - Mechanical Engineering.

It shows that, for the given constant DC linkage voltage, a bigger $\frac{d \psi_{k}}{d \theta}$ will limit the speed. To obtain a wider speed range means a minimized rate-of-change of flux linkage vs. position.

The last but not the least is to evaluate its torque ripple reduction ability. Torque ripple reduction ability is always one of the most important factors to evaluate the TS functions. But there is always a trade-off between the three factors.

A number of TSF has been studied to smooth SRM's torque. Illic-Spong et al. developed an early TSF in [47] exponentially rising and exponentially decreasing, which is called the m-functions in his paper. A linear torque distribution function is proposed by in D.S. schramm et al., which results in a higher torque/current ratio and at the same time widens the speed range [48]. And N. C. Sahoo et al. proposed TS functions of cubic form in [49]. This TSF uses iterative learning to improve the control performance, which makes it of strong robustness, especially when the electromagnetic data of the SRM is limited. Another simple but popular TS function is sinusoidal TSF, which is developed by Husain and Ehsani in [50].

$$
f(\theta)_{i}=\left\{\begin{array}{cl}
0 & \theta_{0} \leq \theta \leq \theta_{o n} \\
0.5-0.5 \cos \left(\frac{\theta-\theta_{o n}}{\theta_{o v}}\right) & \theta_{o n} \leq \theta \leq \theta_{o n}+\theta_{o v} \\
1 & \theta_{o n}+\theta_{o v} \leq \theta \leq \theta_{o f f} \\
0.5+0.5 \cos \left(\frac{\theta-\theta_{o n}}{\theta_{o v}}\right) & \theta_{o f f} \leq \theta \leq \theta_{o f f}+\theta_{o v} \\
0 & \theta_{o f f}+\theta_{o v} \leq \theta \leq \theta_{p}
\end{array}\right.
$$


M.A.Sc. Thesis - Jianing Lin; McMaster University - Mechanical Engineering.

An improved sinusoidal TSF developed in [49] is shown in (4-6) and its signal is shown in Fig. 4.2.

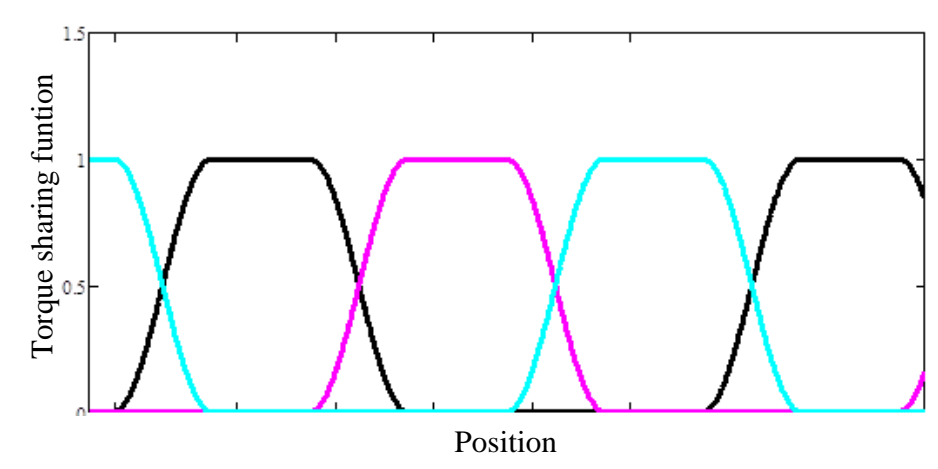

Fig. 4.2. Torque sharing function of three phases.

where $\theta_{p}-\theta_{0}=\frac{2 \pi}{P_{r}}$, which is one rotor period of the designed motor. To avoid the negative torque producing, it is usually suggested to satisfy $\theta_{o f f}+\theta_{o v}-\theta_{0} \leq \frac{\pi}{P_{r}}$, and $P_{r}$ is the rotor pole number. As it is known, the phase difference between adjacent two phases is $\theta_{\text {on }_{-} b}-\theta_{\text {on }_{-} a}=\frac{2 \pi}{P_{r} \frac{P_{s}}{2}}$. And during the period when one phase is fully used to generate torque, torque output for all the other phases should be 0 . That is to say $\theta_{\text {on_ }_{-}}=\theta_{\text {off_a }}$, By putting these two equations together, it can be easily concluded that, $\theta_{o n_{-} b}-\theta_{o n_{-} a}=\frac{2 \pi}{P_{r} \frac{P_{s}}{2}}$. 
M.A.Sc. Thesis - Jianing Lin; McMaster University - Mechanical Engineering.

\subsection{Other Control Strategies}

\subsubsection{Linearization and decoupling techniques}

This control strategy is first proposed by Martija Ilic'-Spong in [52]. It used a state feedback control algorithm, which compensated for all the nonlinearities by matching the model with experiment data. By translating the coupled nonlinear model into a simple decoupled linear system, traditional control techniques will be valid to generate a high quality performance in SRM. However, to realize this state control technique requires that all the state variables are testable, like current, position, and velocity, which definitely increase its cost.

\subsubsection{Sliding mode variable structure control}

Sliding mode control is also a state feedback control strategy, which forces the system to slide along a special cross-section of the system's state and finally get stable. SMV is more robust, better dynamic response ability and easy to implement, compared with LDT. However, it will introduce high frequency chatter and therefore increase SRM's torque ripple [53].

\subsubsection{Other control strategies}

As for SRM's control strategies, it must be able to reduce its torque ripple and noise, as these are the biggest problems hindering SRM's development. At the same time, it should have better dynamic performance, easy implementation and robustness. Thus, some intelligent control algorithms, like fuzzy-logic-based [54] and neural-network-based techniques [55], [56], are also studied on SRM. They both have good self-learning and 
M.A.Sc. Thesis - Jianing Lin; McMaster University - Mechanical Engineering.

adaptive capability. However, they are still time and money-costing. Further developments are still required to apply these intelligent control strategies in real industry.

\subsection{APC vs. TSF in Matlab/Simulink}

The nonlinear model of the designed SRM in Matlab/Simulink is shown in Fig. 4.3, based on the FEA in chapter 3. The inductance-position-current look-up table and torqueposition-current look-up table are used in the model.

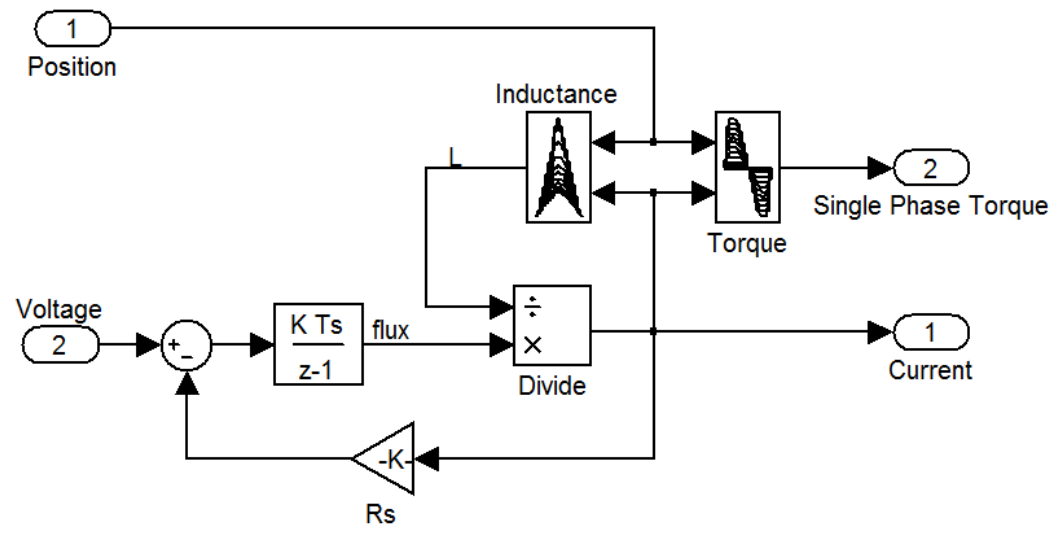

Fig. 4.3. SRM modeling in Matlab/Simulink.

Data of the two look-up table both comes from the 2D FE analysis of JMAG. Detailed condition setting, careful meshing sensitivity checking and step setting are conducted during the FE analysis to make sure its accuracy.

\subsubsection{Turn-on/off angle control (angular position control)}

Angular position control is one of the most classic control strategies for SRM. The idea of APC is by defining the turn-on and turn-off angles of each phase. If the position is within this range, this phase will be turned on, otherwise it is off. The simulation diagram is shown in Fig.4.4. 


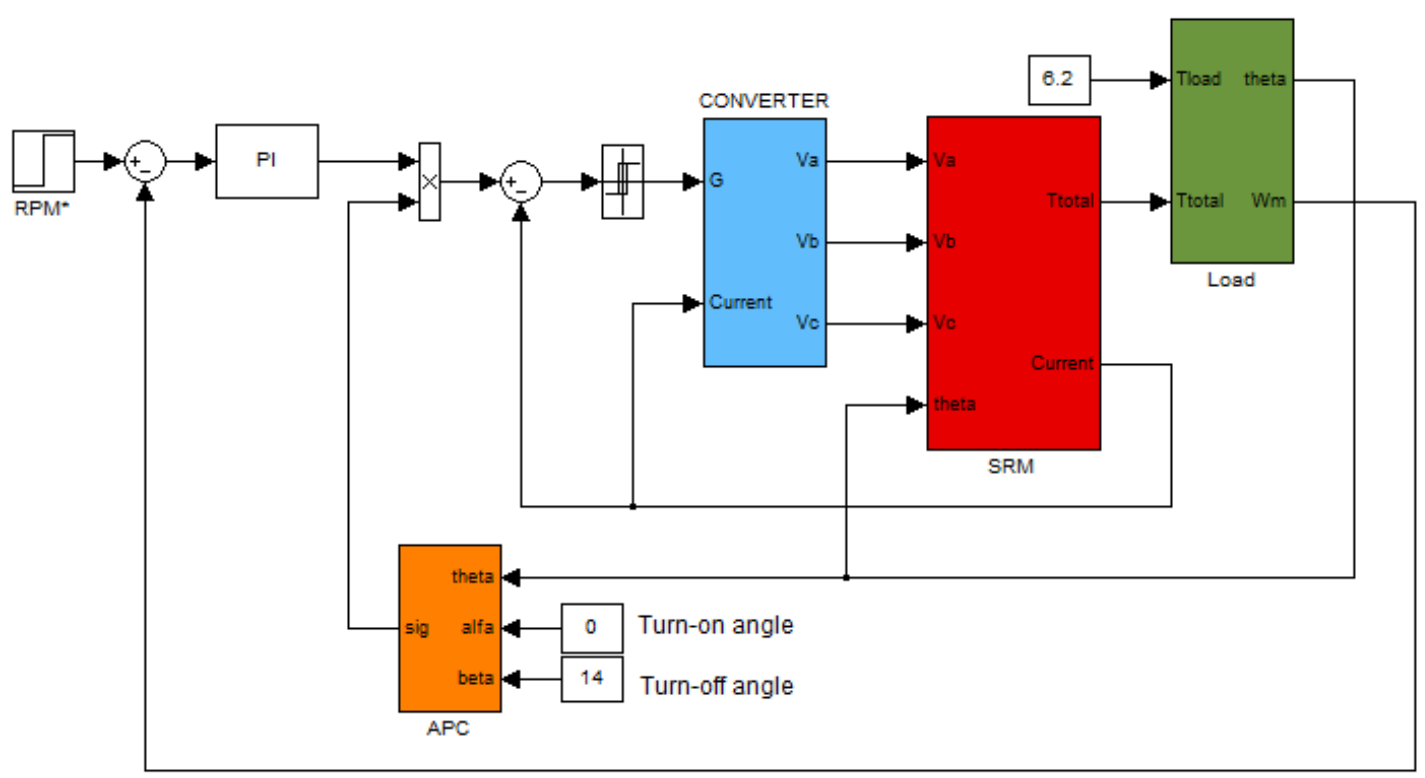

Fig. 4.4. Angular position control diagram in Matlab/Simulink.

As the load torque is time-varying as mentioned in chapter 3. An average load torque of 6.2 $\mathrm{Nm}$ is chosen in Matlab/Simulink. The reference speed is set to $500 \mathrm{rpm}$ from 0 at the time of 0.1 second. The required reference current is restricted within $35 \mathrm{~A}$, and the bandwidth of hysteresis current regulator is between $-1 \mathrm{~A}$ and $1 \mathrm{~A}$.

Two issues are to evaluate the control strategy's performance. One is its starting ability. To some extent, the starting torque should be as big as possible. The second one is torque ripple reduction. The torque ripple at rated speed is studied as one of the optimizing objectives of this thesis.

By changing the turn-on or turn-off angle, the machine's output performance varies. In this thesis, the starting torque is set to be the average torque from the time motor to accelerate, 0.1 second, to the time of 0.3 second, which is defined as the starting period for the motor. 
M.A.Sc. Thesis - Jianing Lin; McMaster University - Mechanical Engineering.

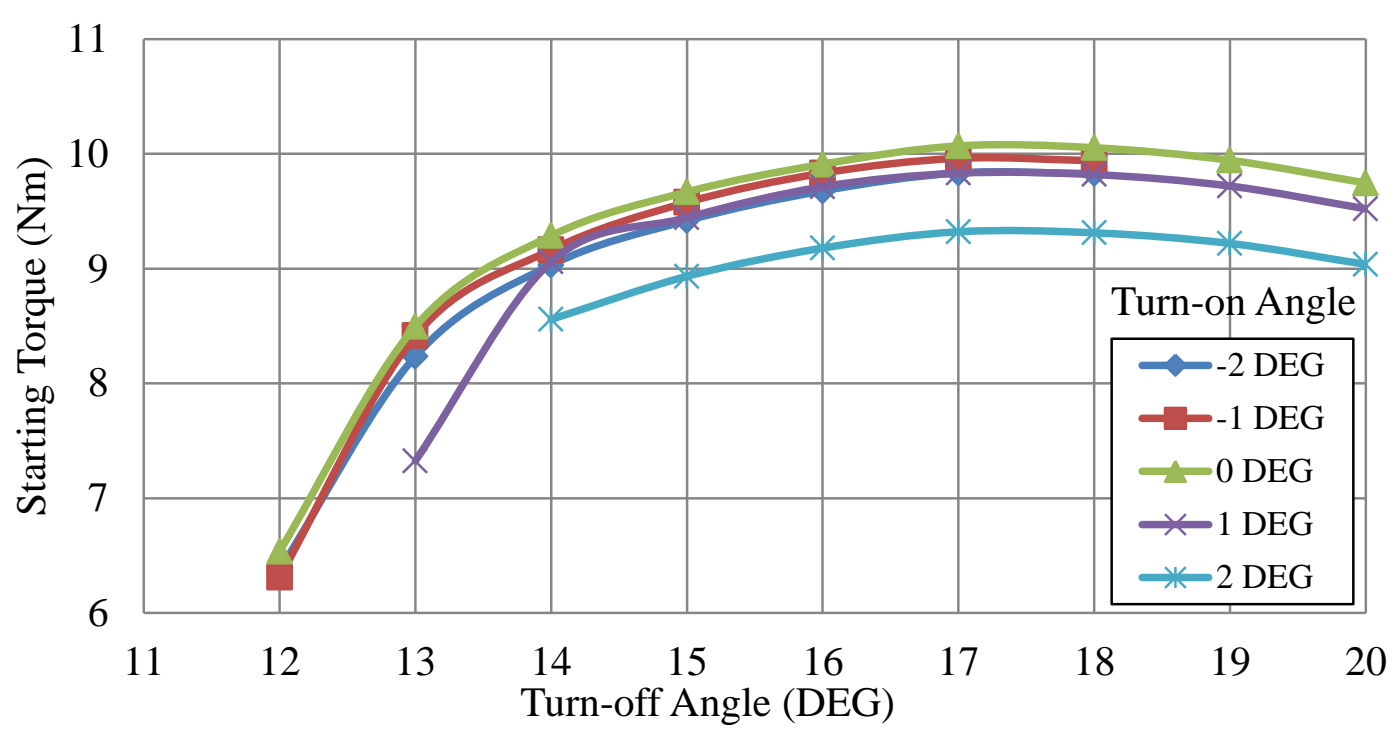

Fig. 4.5. Starting ability with different turn-on and turn-off angles.

As shown in Fig. 4.5, the turn-on angle has very limited affection on the starting torque especially for those smaller turn-on angles. For the turn-on angle of -2 degree, -1 and 0 degree, the starting torque rises slightly with the turn-on angle increasing. However, when the turn-on angle keeps increasing and getting larger than 0 degree, the average starting torque begins to drop. The decrease is much more significant. For a constant turn-on angle, the starting torque versus turn-off angle curve increases firstly with a high slope. After reaching to the highest value, it starts to move downward. That is to say, by increasing the turn-off angle, the overlap period rises, and the starting torque keeps increasing. However, it reaches the peak when the turn-off angle is between 17 and 18 degree, because that after the position of 18 degree the machine will generate negative torque. A little advanced turnoff angle than 18 degree is necessary to make sure the current drop back to 0 before negative torque generates. It is the same with a minus turn-on angle, which will decrease the average starting torque, as it introduces negative torque. However, as the current takes 
M.A.Sc. Thesis - Jianing Lin; McMaster University - Mechanical Engineering.

time to rise, the average torque drop isn't that significant. The highest starting torque is with the turn-on angle of 0 degree and turn-off angle of 17.625 degree in this study. The best starting torque is $10.08 \mathrm{Nm}$.

However, the torque ripple profile shows a different conclusion, as seen in Fig. 4.6. With the turn-on angle increasing, the torque ripple vs. turn-off angle curve moves rightwards and upwards in general. For the same turn-off angle, an earlier turn-on angle has better influences on the output torque ripple, which also implies a bigger overlap period. However, there is also intersection between curves, as seen in the Fig above. That is to say, the general principle doesn't work all the time, for a certain turn-off angle, a latter turn-on angle may be better for the torque ripple reduction.

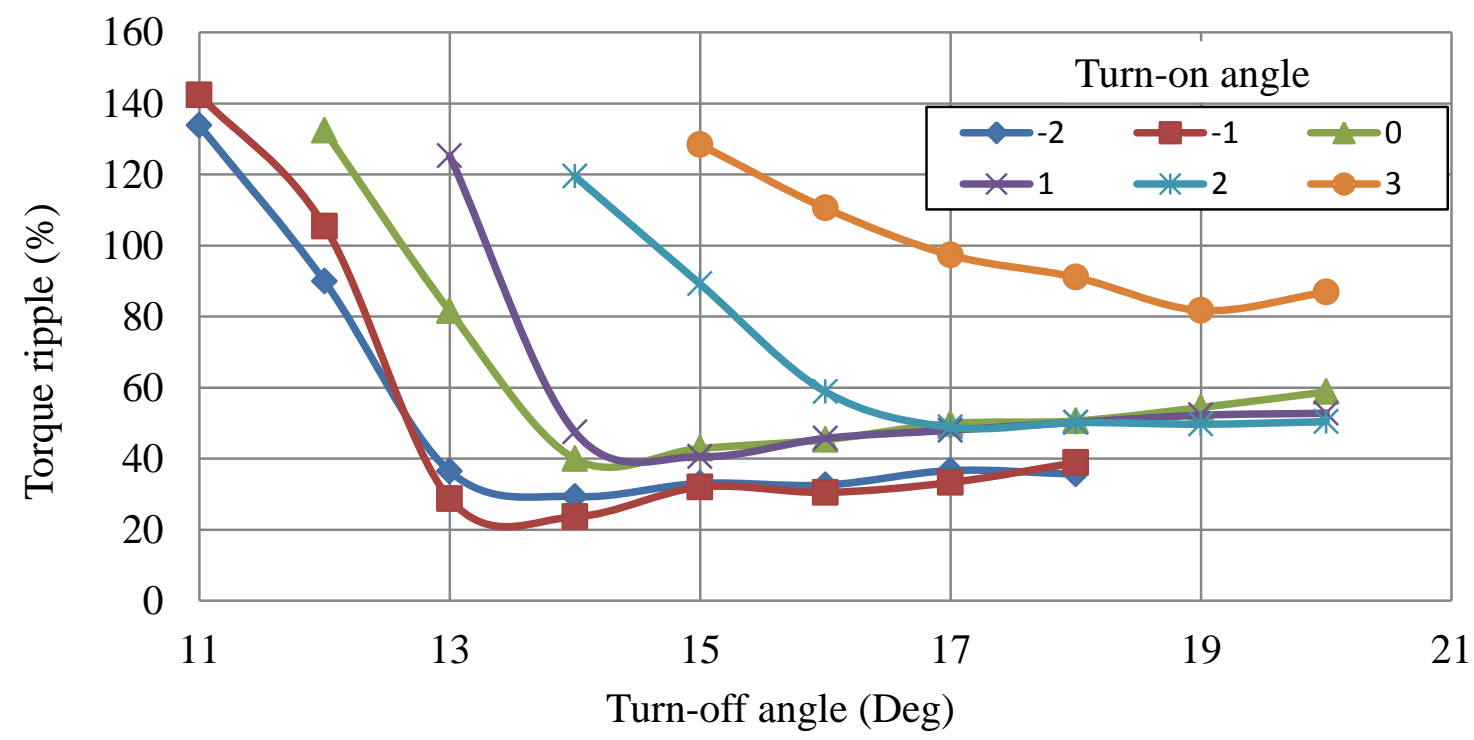

Fig. 4.6. Torque ripple at rated speed with different turn/off angles.

For a certain turn-on angle, the torque ripple decreases significantly as the turn-off angle increases for a smaller turn-off angle. However, the decrease stops after certain 
M.A.Sc. Thesis - Jianing Lin; McMaster University - Mechanical Engineering.

value and it starts to increase, but with a small the increasing slope. In another word, after a certain turn-off angle, the torque ripple reduction is harder to improve by increasing turnoff angle. The best reduced torque ripple is of $23.64 \%$ with the turn-off angle of 14 degree, and turn-on angle of -1 degree.

From Fig. 4.5 and Fig. 4.6, it is concluded that there is a trade-off between the average torque and torque ripple. In this thesis, more attention is paid to the torque ripple reducing, so there has to be a decrease of output average torque. The torque and current profile for the best torque ripple reduction at rated speed is shown in Fig. 4.7.
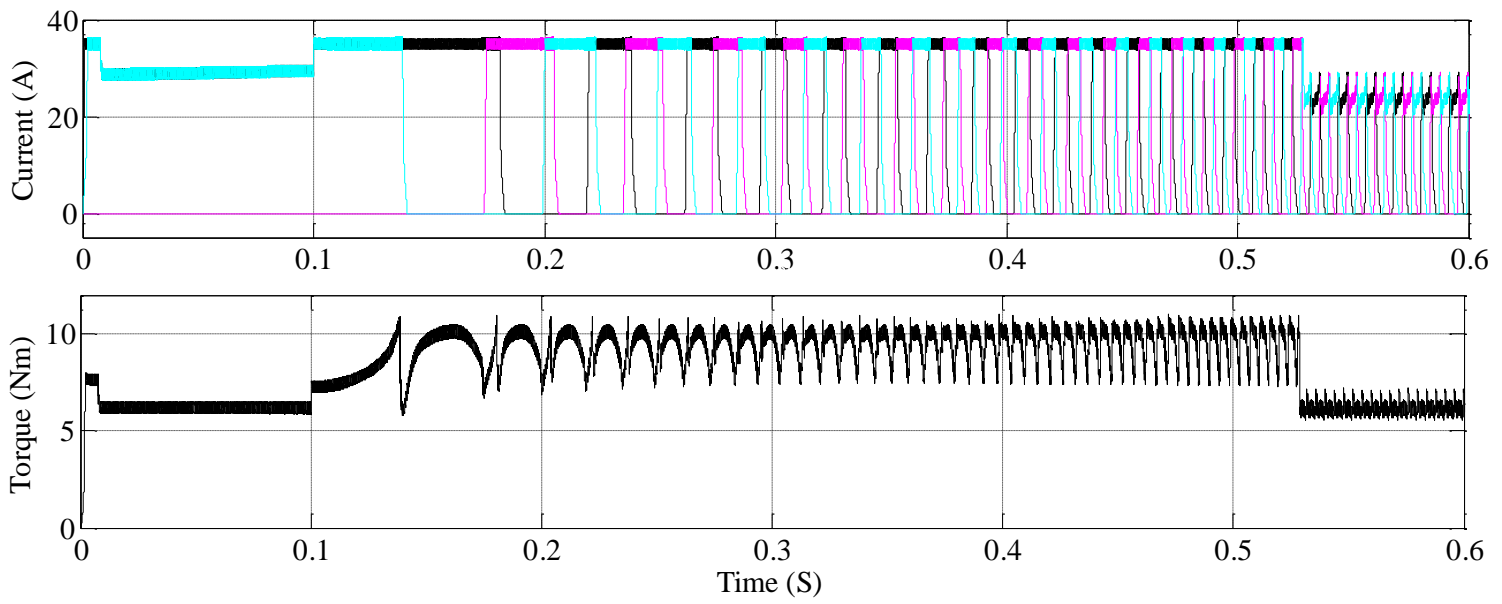

Fig. 4.7. Current and torque profile with turn-on angle of -1 degree and turn-off of 14 degree.

As shown, the motor is static at the beginning, and starts to accelerate when the reference speed jumps to $500 \mathrm{rpm}$ at the time of 0.1 second. During the accelerating period, the required torque, calculated by the PI controller is bigger and the currents are all the maximum value of 35A. And it reaches the rated speed at the time of 0.529 second. Then 
M.A.Sc. Thesis - Jianing Lin; McMaster University - Mechanical Engineering.

the required torque reduces to about $6.2 \mathrm{Nm}$, which is the required load torque. A detailed figure for the torque performance at rated speed is shown in Fig. 4.8.
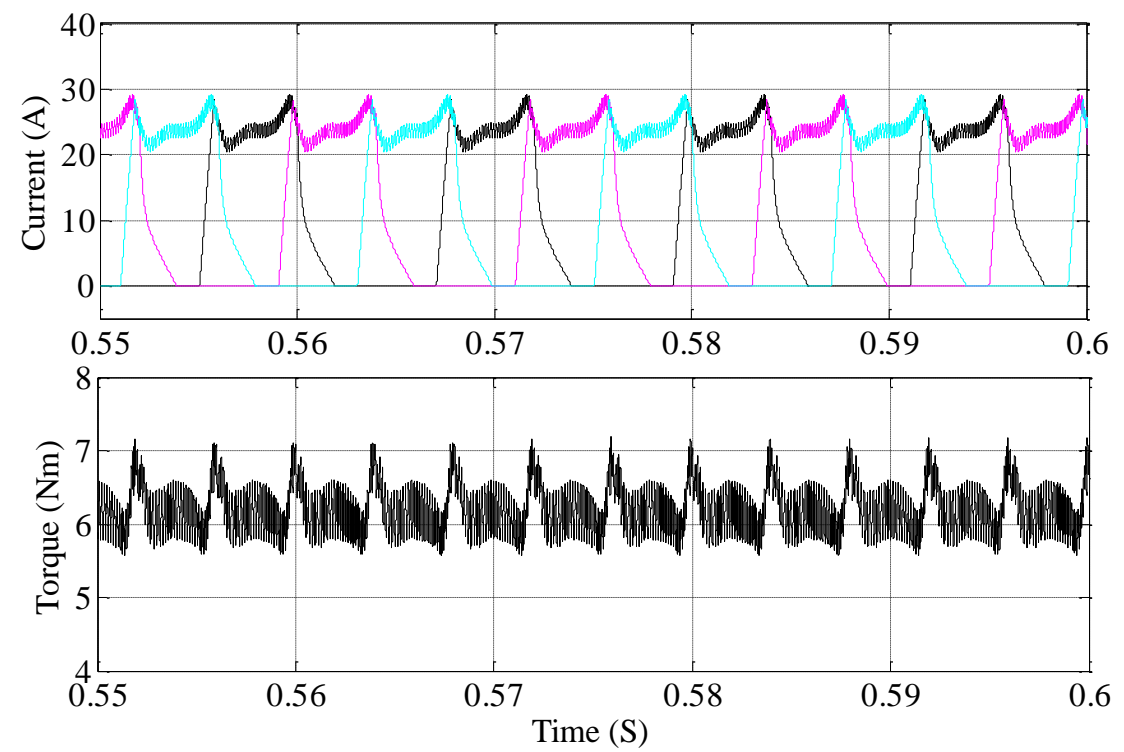

Fig. 4.8. Current and torque profile at rated speed with turn-on/off of -1 and14 degree.
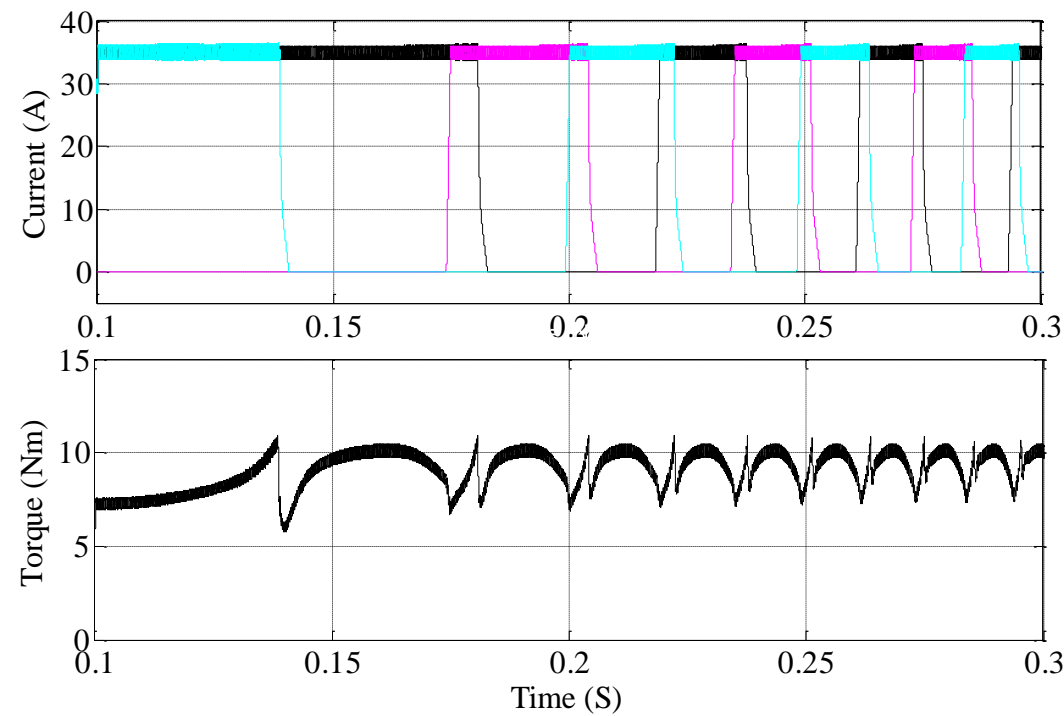

Fig. 4.9. Current and torque profile during accelerating with turn-on/off of -1 and14 degree. 
M.A.Sc. Thesis - Jianing Lin; McMaster University - Mechanical Engineering.

The torque ripple happens at the commutation period. During the commutation period, the turn-on phase usually has much smaller induction than the turn off phase, so it will respond quickly. However, the turn-off phase reacts much slower. They work separately to generate the torque and un-required torque ripple happens. By optimizing turn-on and turn-off angles, torque ripple at rated speed can be reduced to $23.64 \%$, which is an acceptable level.

For the accelerating period, the torque ripple is much bigger, as shown in Fig.4.9. However, the torque ripple reduction is especially important at the lower speed range. Thus, another torque ripple study is conducted to find out an optimal turn-on and turn-off angle at the low speed (accelerating) period. In this thesis, a lower speed range is defined as 100 to $200 \mathrm{rpm}$. Fig. 4.10 shows the relationship between the torque ripple and turnon/off angle at lower speed range.

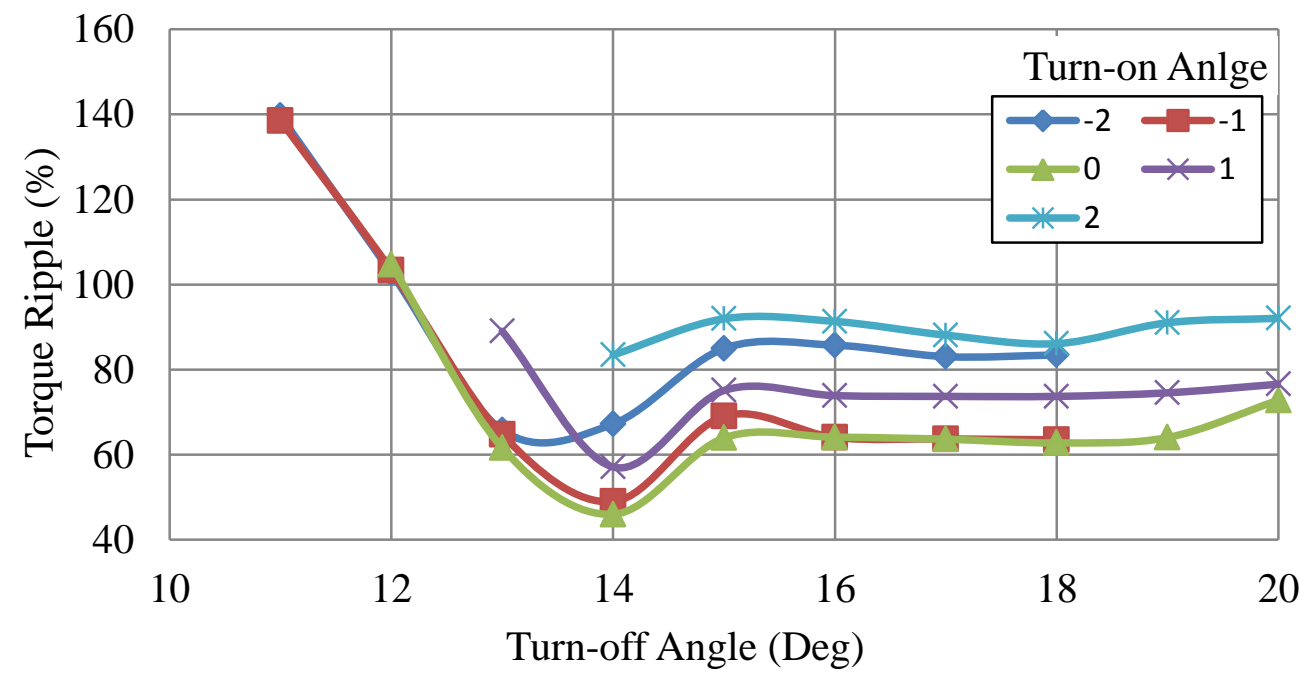

Fig. 4.10. Torque ripple at lower speed range with different turn/off angles. 
M.A.Sc. Thesis - Jianing Lin; McMaster University - Mechanical Engineering.

It shows a different feature with the torque ripple at rated speed. In general, the curve will move downwards as the turn-on angle increases but less than 0 degree. However, it changes to move upwards after turn-on angle reaches positive. That means, the torque ripple decreases first and then increases as the turn-on angle gets bigger for the accelerating period. For a certain constant turn-on angle, the motor's torque ripple will first decrease with the increase of turn-off angle, but turn to increase after some certain value, and then become almost constant when keeping increasing the turn-off angle. It has been found out that the best turn-on and turn-off angles for the reduction of torque ripple at low speed range is with turn-on angle of 0 degree, and turn-off angle of 14 degree. The torque ripple is about $45.99 \%$, as shown in Fig. 4.11.

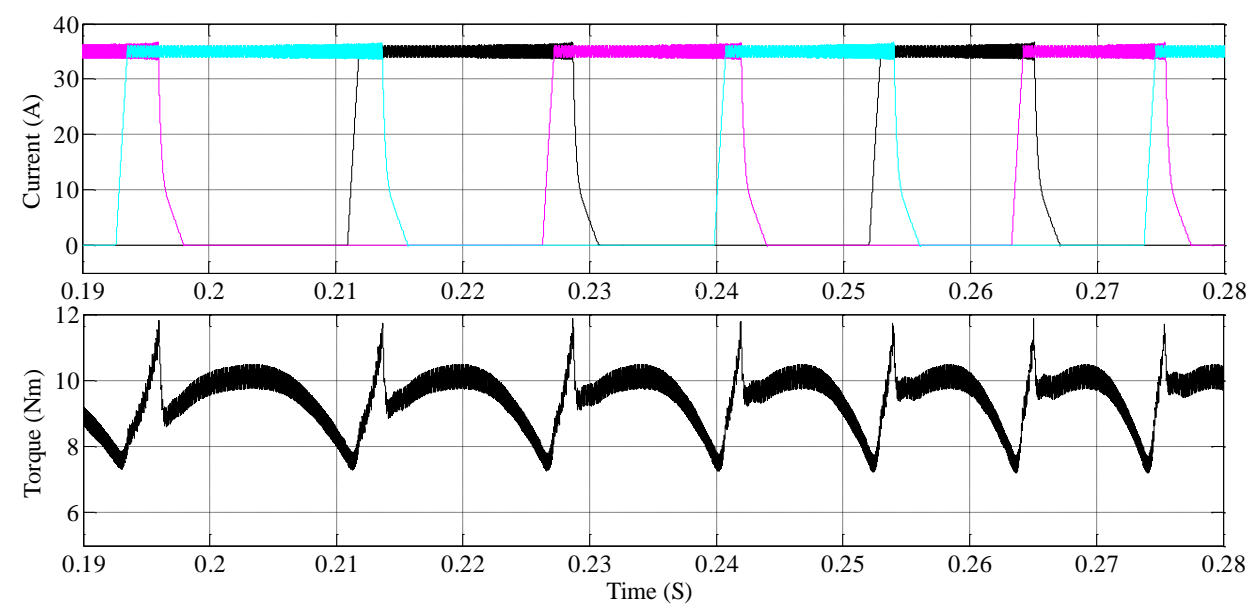

Fig. 4.11. Current and torque profile at lower speed range with turn-on/off of 0 and14 degrees.

To conclude, the turn-on turn-off angle control is not a good solution for varying speed. A certain turn-on and turn-off angle may be only suitable for a certain speed. It is better to find out a time-varying turn-on and turn-off angle for the future work. At the same time, 
M.A.Sc. Thesis - Jianing Lin; McMaster University - Mechanical Engineering.

the study above shows that the torque ripples can be reduced to $23.64 \%$ for the rated speed, but only $45.99 \%$ for low speed range.

\subsubsection{Torque sharing function control strategy}

Overlap period between phases has very good affection on the torque production as well as the torque ripple reduction especially at lower speed range, however, as the overlapped two phases generate the torque independently, the two phases both generate torques during the overlap period, and the sum of the two phases' torque will increase significantly, which will lead to big torque ripple. Hence, the torque sharing function control is introduced to improve the torque ripple, which introduces the sharing function to assign the desired torque for each phase during overlap period.

The control configuration is shown in Fig 4.12. The orange block in 4.12 will assign the desired torque to each phase based on the torque sharing function pre-defined. Then it's to find out the required current to generate the desired torque at certain position. Taking the system's nonlinearity, the 2D look-up table with input of position and assigned torque and output of required current is used.

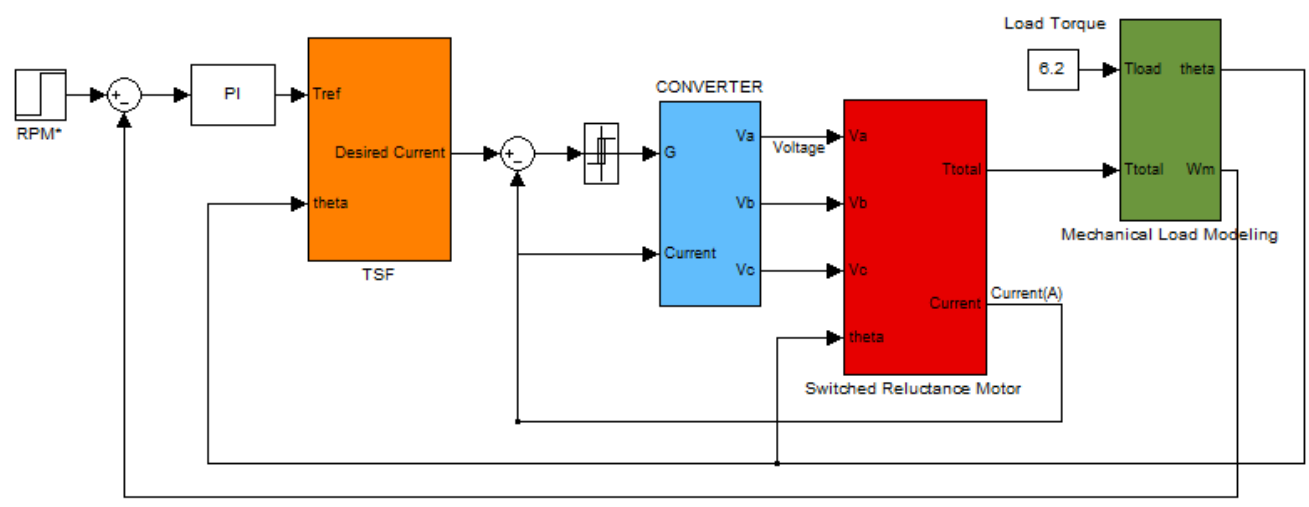

Fig. 4.12. Torque sharing function control diagram. 
M.A.Sc. Thesis - Jianing Lin; McMaster University - Mechanical Engineering.

The 2D lookup table is calculated from the FEA result of JMAG. However, there is only the relationship from current and theta to torque. Fitting functions are used to get the relationship inverted to get the desired current from the input of position and torque.

A higher bandwidth of current regulator is necessary to regulate the phase current, which in turn, maintains the desired phase torque instantaneously. So to study the influence of torque sharing block, it's simply assumed that the power source is able to generate the desired current and the power electronic is also ideal, but within 120A, which is the maximum current calculated in FEA.

For the sharing function in (4-6), $\theta_{0}$ is defined as the exactly unaligned position. With the position angle increasing, the rotor moves from unaligned position to aligned position, and then unaligned position. $\theta_{p}$ is defined as the aligned position. For all the variations, there are only two independent variations, and all the others can be calculated based on these two. One is turn-on angle $\theta_{o n}$, and the other is the overlap angle $\theta_{o v}$.

The starting torque curves are shown in Fig. 4.13. It shows that starting torque has very little change during the turn-on angle and overlap angle changing. Except the one with turn-on angle of 0 degree and overlap angle of 1 degree, all the others are between $10.7 \mathrm{Nm}$ to $11.1 \mathrm{Nm}$. For the smaller overlap angle range, a latter turn-on angle is preferred for a big starting torque. Otherwise, its inductance increasing slope will be too small for the required starting torque. However, with the overlap angle increasing, an earlier turn-on angle is suggested. As a big overlap angle with a latter turn-on angle will lead to a late turn-off angle. So, there are intersections between the starting torque curves. For a constant 
M.A.Sc. Thesis - Jianing Lin; McMaster University - Mechanical Engineering.

turn-on angle, the starting torque rises with the overlap angle with bigger slop, but then climbs slowly, and finally starts to slightly fluctuations.

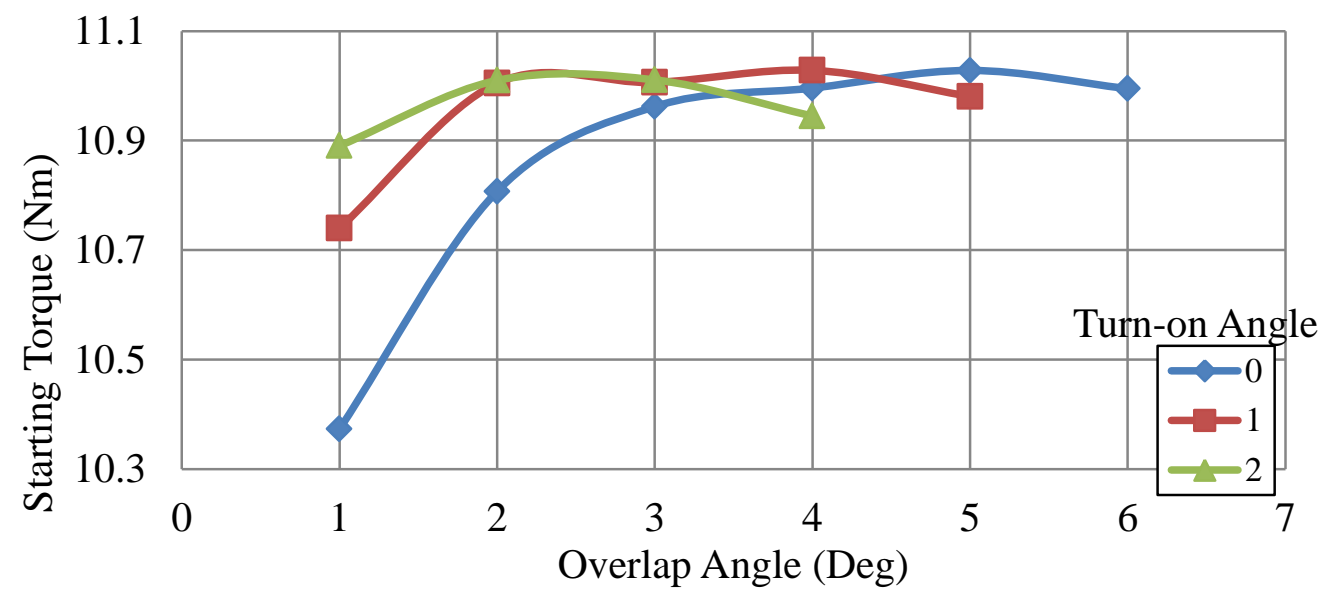

Fig. 4.13. Starting torque with TSF control.

Besides the starting torque, the torque ripple at lower speed range of $100 \mathrm{rpm}$ to 200 rpm is shown in Fig. 4.14. It's concluded that, the curve moves leftwards and downwards in general. But there are intersections between curves. That's to say, the starting torque ripple for $100 \mathrm{rpm}$ to $200 \mathrm{rpm}$ is decreasing as the turn-on angle increases for smaller overlap angles. However, with the overlap angle keeps increasing, the torque ripple changes to increase with the turn-on angle. For a constant turn-on angle, the torque ripple decrease very significantly as the overlap angle increases, however, the decreasing slope slows down after certain overlap angles. It shows those overlap angles vary with the turnon angles, however, they are leads to a turn-off angle of 14 degree. That's to say, for the designed SRM with TSF control, the torque ripple reduction isn't that significant with turnoff angle larger than 14 degree. 
M.A.Sc. Thesis - Jianing Lin; McMaster University - Mechanical Engineering.

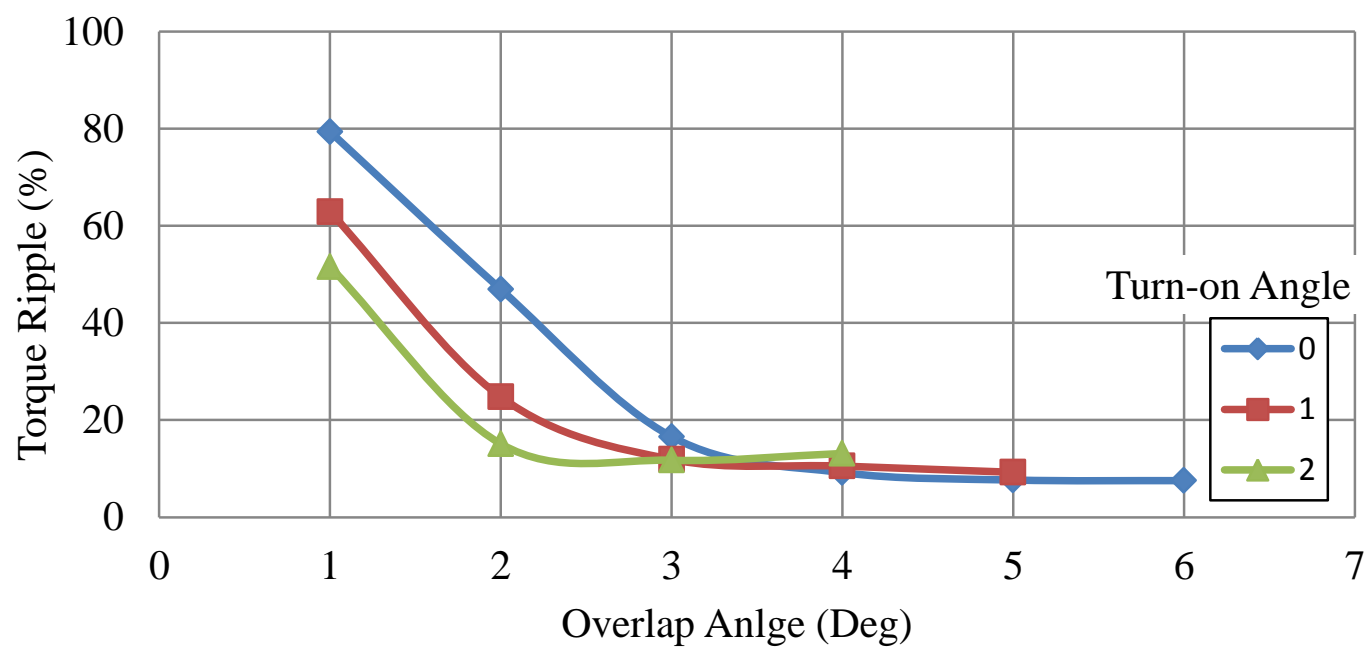

Fig. 4.14. Torque ripple at lower speed range with TSF.

Detail current and torque profiles with a constant but smaller overlap angle of 1 degree, and different turn-on angles are compared in Fig. 4.15. It shows that, to track the required torque, an earlier turn-on angle will introduce a big current jump at the beginning, however a later one will leads to a latter turn-off angle, which also introduce a current jump, but at the end of the energizing period. Turn-on of 2 degree and overlap of 1 degree shows a better torque ripple reduction, however at a big expense of current oscillation.

At the same time, the current and torque profile with the constant but larger overlap angle, but different turn-on angles is also compared in Fig. 4.16. The comparison shows that, a big overlap angle plus a late turn-on angle will lead to a very late turn-off angle, which will introduce very big current jump at the turning-off region, which will then lead to higher losses, and sometime dangerous for the power electronics. 
M.A.Sc. Thesis - Jianing Lin; McMaster University - Mechanical Engineering.
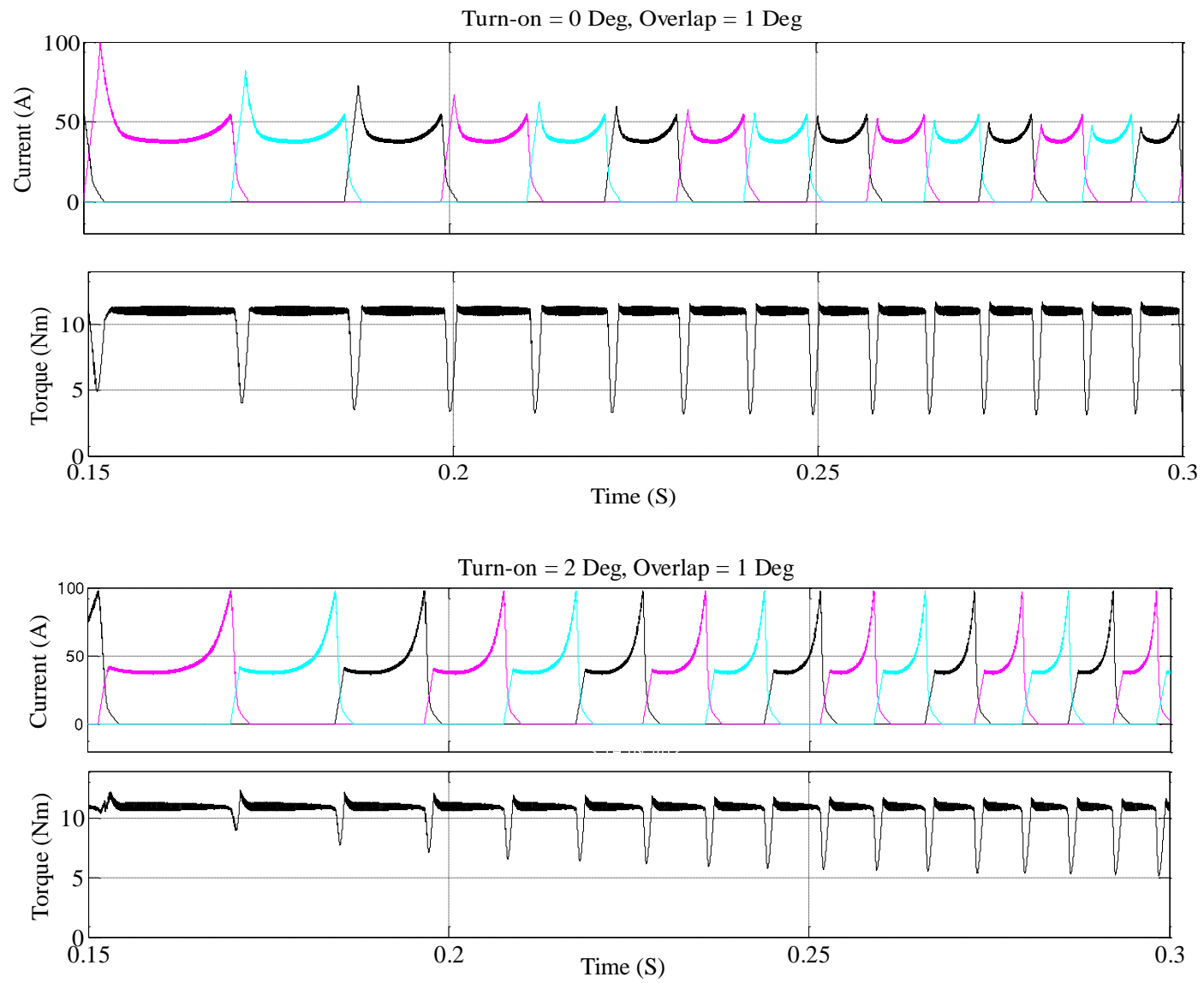

Fig. 4.15. Comparison of current and torque profiles with smaller overlap angle but different turn-on angles.

After all, the best reduced torque ripple for this designed SRM with TSF control is $7.53 \%$, within $10 \%$. The turn-on angle is 0 degree, and overlap angle of 6 degree, which is shown in Fig. 4.17. The torque ripple at rated speed is studied in Fig. 4.18. The optimally reduced torque ripple for the rated speed range here is $15.49 \%$ with turn-on angle of 0 degree and overlap of 6 degree as shown in Fig. 4.19. 
M.A.Sc. Thesis - Jianing Lin; McMaster University - Mechanical Engineering.
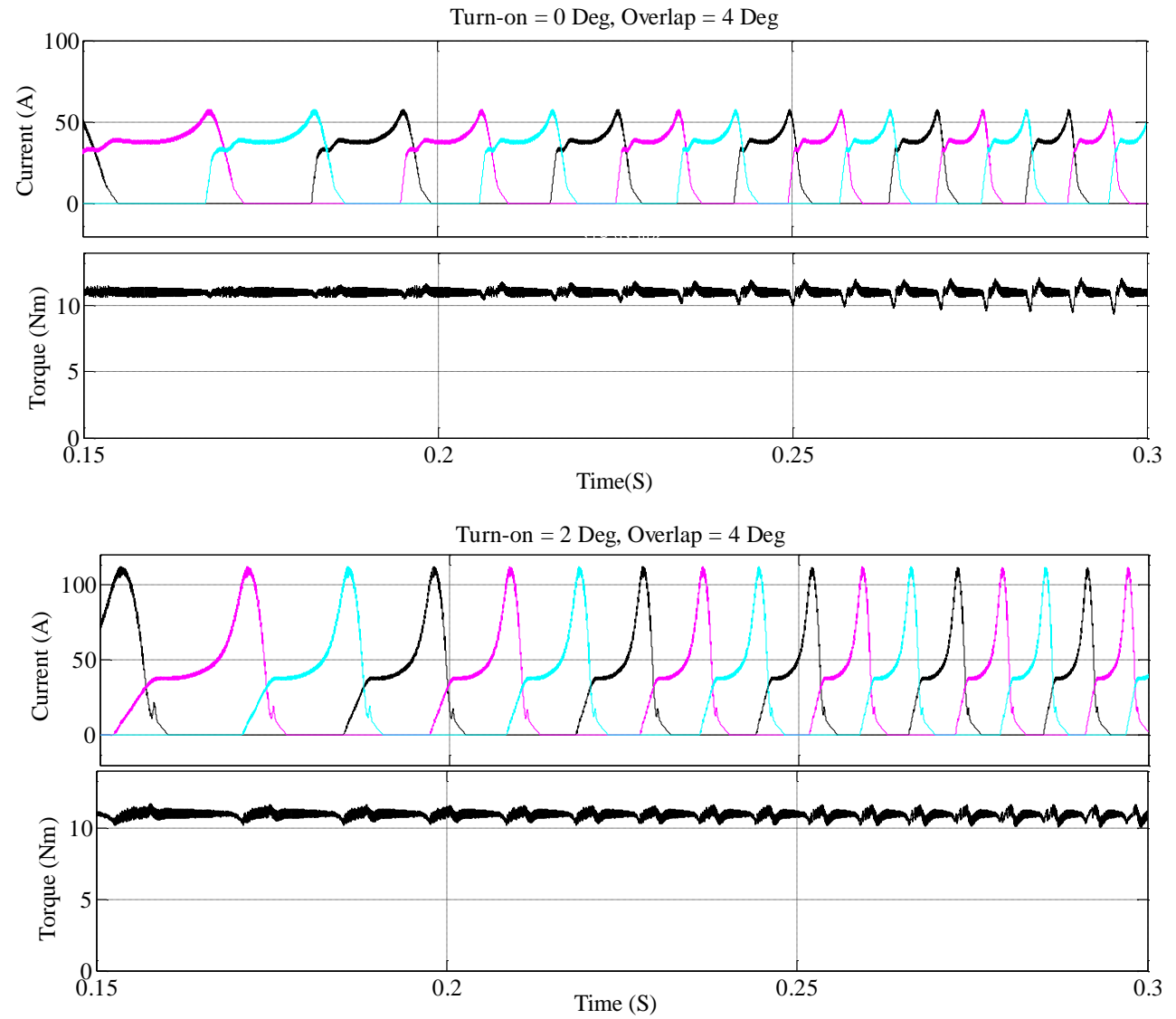

Fig. 4.16. Comparison of current and torque profiles with larger overlap angle but different turn-on angles.

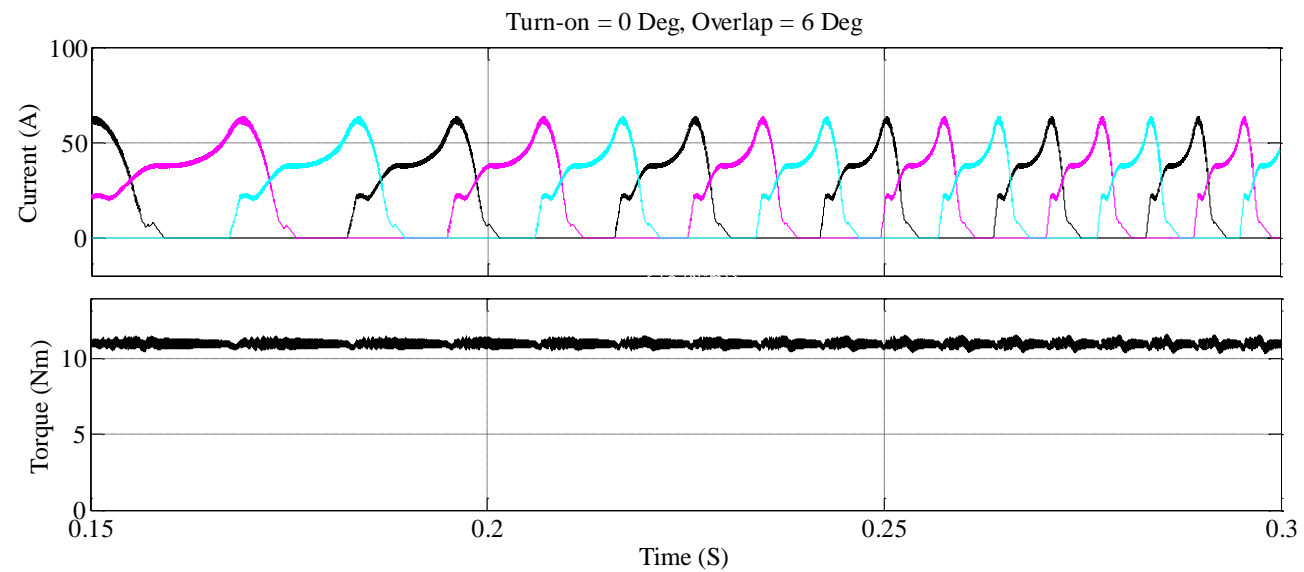

Fig.4.17. Current and torque profile with turn-on/overlap of 0 and 6 degrees for TSF control. 
M.A.Sc. Thesis - Jianing Lin; McMaster University - Mechanical Engineering.

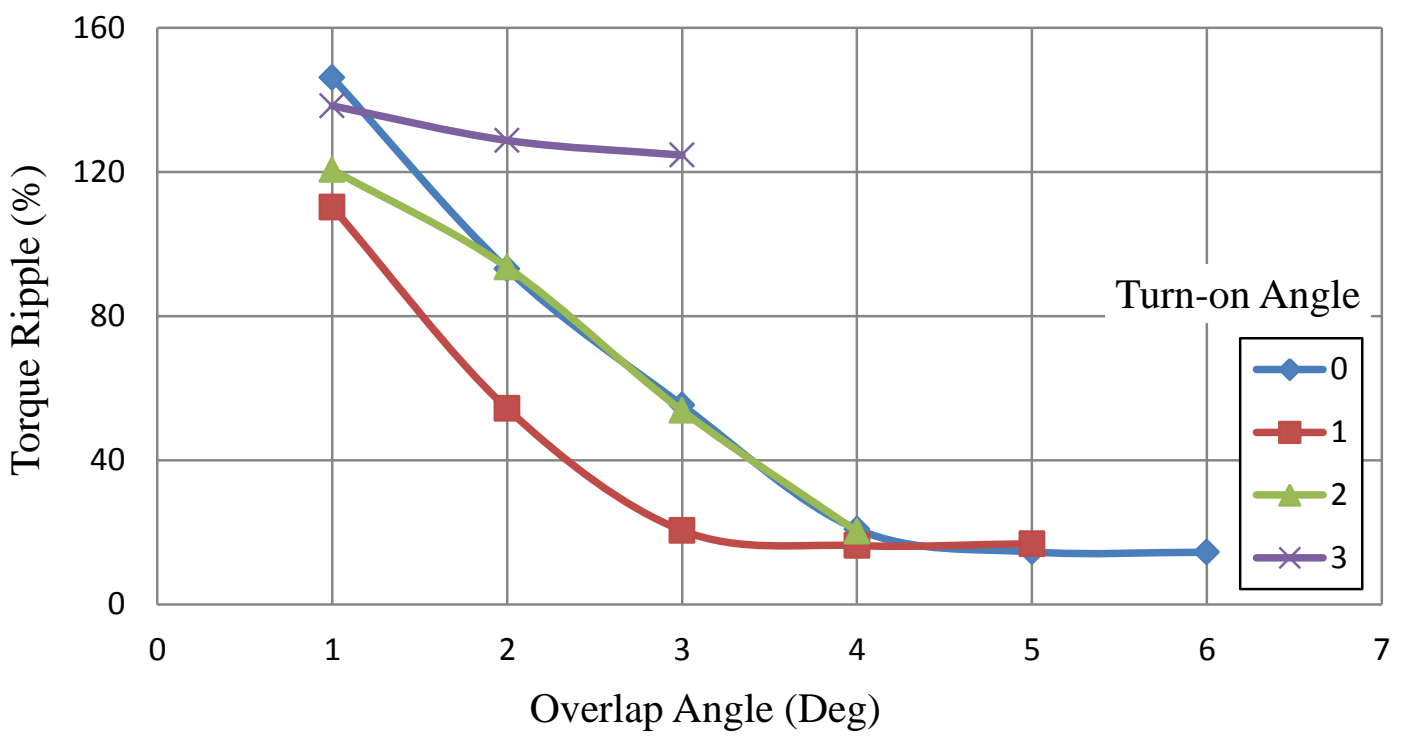

Fig. 4.18. Torque ripple at rated speed with TSF control.

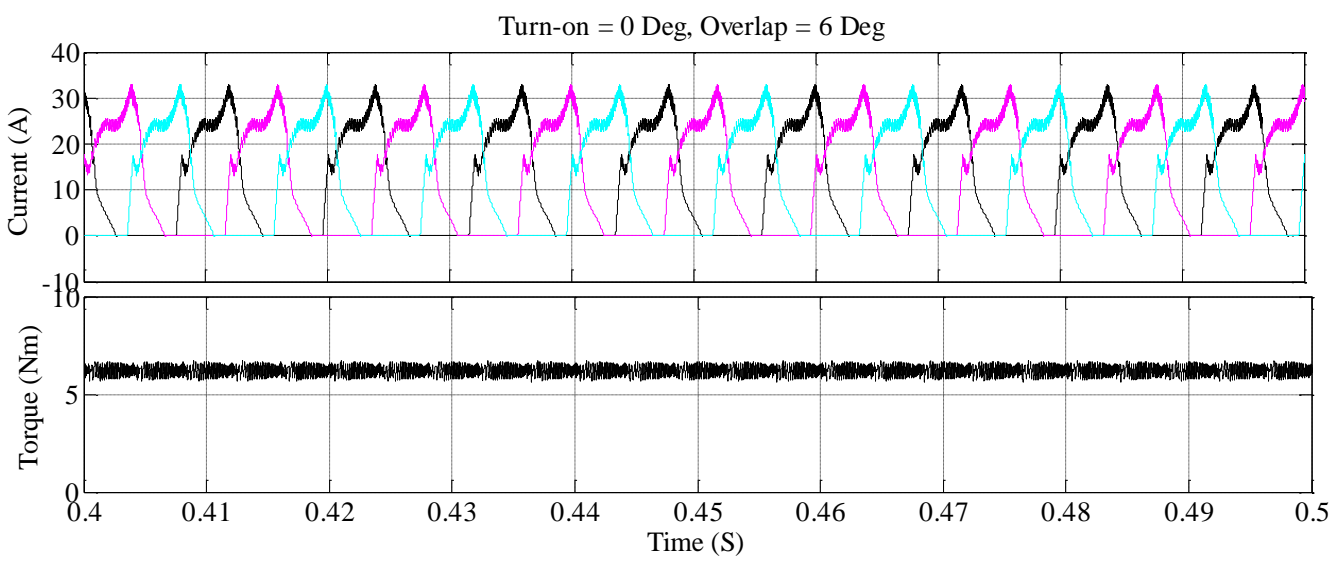

Fig. 4.19. Current and torque profile at rated speed with TSF.

It's clear that the TSF has better starting ability and torque ripple reduction than APC. However, this is at the expense of bigger current and losses. 
M.A.Sc. Thesis - Jianing Lin; McMaster University - Mechanical Engineering.

\subsection{Conclusions}

The doubly salient structure and nonlinearity of SRM, resulting from its deep saturated operation condition, make its torque ripple more serious than other machines. Two torque ripple reduction control strategies are conducted and compared in this chapter. The angular position control can reduce the torque ripple to $23.64 \%$ for the rated speed, but only $45.99 \%$ for low speed range. And it can be achieved to $7.53 \%$ for acceleration speed range, but $15.49 \%$ at rated speed with torque sharing function control, however at an expense of higher current and larger copper losses. 
M.A.Sc. Thesis - Jianing Lin; McMaster University - Mechanical Engineering.

\section{Chapter 5 Thermal Analysis of Designed SRM}

For the designed SRM, as an external-rotor and internal-stator electric machine, it has advantage of simple structure, reduced torque ripple. In addition, the external rotor structure makes it easy for in-wheel mechanical manufactory and assembly. However, its internal stator structure also limits the heating radiating out compared with a normal external stator one. Especially for a 35A current, the temperature increases more significantly. This will further result in poor machine performance, also destroy the coil insulation, reducing winding life and leading to safety problem. Thus, thermal analysis is conducted in this chapter to predict and avoid those problems.

In this chapter, a detail 3D finite element thermal analysis is conducted to predict the temperature variation and distribution of the designed SRM, both at rated operation condition and also for taking into consideration of velocity cycle.

\subsection{Thermal Analysis Process}

One of the most important reasons to handle the generated heat is increasing motor's efficiency, also with higher performance. Therefore, to obtain the thermal flow of the designed SRM is the objective of this project. FEA is chosen here for the thermal analysis of this motor and JMAG is used [57].

As known, the temperature variation is based on the generated heat, which is the copper and iron loss for this SRM. Copper loss is much easier to calculate, but the iron loss is related to the magnetic result. Thus the magnetic result will be got first and then used for 
M.A.Sc. Thesis - Jianing Lin; McMaster University - Mechanical Engineering.

the iron loss analysis. Thus, thermal analysis for each element is conducted by coupling the magnetic result together with the thermal analysis.

Normally there are two coupling ways, one-way and two-way as shown in Fig. 5.1. It's easy to tell that the two-way coupling is more accurate since it also includes the affection of temperature variation on magnetic performance. However, two-way coupling is much more complex and takes much longer time and more computer resource. Thus the one-way coupling is more widely used.
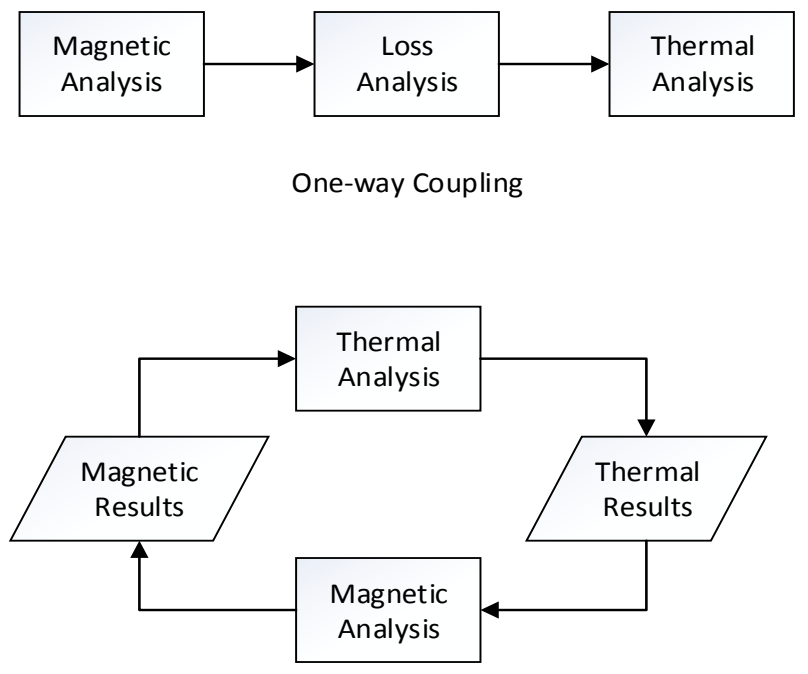

Two-way Coupling

Fig. 5.1. Coupling ways of thermal finite element analysis.

As for the magnetic analysis, 2D model is enough to get an accurate results and it saves computer time. However, 3D model is required for thermal analysis in JMAG.

\subsection{Loss Analysis at Rated Condition}

Temperature variation results from the generated losses of the motor. That's to say, calculating the losses become the first step for the whole process. The heat generation in 
M.A.Sc. Thesis - Jianing Lin; McMaster University - Mechanical Engineering.

the electric motors can be divided in four groups, Joule losses, iron losses, stray load losses and mechanical losses.

Joule losses is directly related to the electric resistance of the conductor and changes proportionally to the square of the current. This kind of heat occurs in the copper windings, which locates in the stator.

Iron losses are due to the conversion of electric energy into thermal energy in the iron. They are divided in hysteresis and eddy currents losses. The eddy-current losses are Joule losses that occur in the iron due to the flow of an induced electric current. The hysteresis losses are due to the energy expended to align the iron magnetic poles to the applied magnetic field and their order of magnitude corresponds to the area of the hysteresis loop [58].

The stray load losses are minor losses in the electric motor operation and their quantification is very difficult. They include the losses due to the skin effect, high frequency, among others, that are unknown or not easily quantified.

Mechanical losses comprise the conversion of the mechanical energy into thermal energy due to mechanical friction and viscous losses.

In this thesis, only the joule losses and iron losses are considered as the heat source. Losses at rated condition are shown in Table 5.1.

Table 5.1. Losses and efficiency at rated condition.

\begin{tabular}{c|c|c|c}
\hline Stator Iron Loss (W) & Rotor Iron Loss (W) & Copper Loss (W) & Efficiency (\%) \\
\hline 16.67 & 23.3 & 68.6 & 78 \\
\hline
\end{tabular}


M.A.Sc. Thesis - Jianing Lin; McMaster University - Mechanical Engineering.

To improve the efficiency, losses need to be reduced. However, the iron losses are related to the flux density and its variation frequency and the decrease of this will also change the motor's other output performance. Analysis here is trying to avoid this complexity.

For the copper loss, there are two ways to reduce the loss. One is to decrease the current value and the other is to reduce the electrical resistance. As the reduced current causes a significant drop of electromagnetic torque, electrical resistance is chosen to improve the efficiency at last. As discussed above, the filling factor for AWG 9 is 0.54 within the recommend constrain 0.6. However, for the special internal stator structure, it is easier to add the windings. In fact, the geometry between stator poles decreases the winding difficulty. Therefore, AWG 8 is also acceptable for the winding, with a filling factor of 0.64. The copper loss is then reduced to $34 \mathrm{~W}$. The efficiency is improved to $86 \%$, as shown in Table 5.2.

Table 5.2. Losses and efficiency comparison for different winding.

\begin{tabular}{l|l|l|l}
\hline AWG & Copper Loss $(\mathrm{W})$ & Total Loss $(\mathrm{W})$ & Efficiency \\
\hline 11 & 68.6 & 108.6 & $78 \%$ \\
\hline 9 & 43.0 & 83.0 & $84 \%$ \\
\hline 8 & 34.1 & 74.1 & $86 \%$ \\
\hline
\end{tabular}

\subsection{Average Loss Calculation Considering the Velocity Cycle}

\subsubsection{Copper losses}

By integrating equation (2-10) on mechanical angle, it can calculate the average torque from unaligned position to aligned position, as the whole rotation process is the repeat of 
M.A.Sc. Thesis - Jianing Lin; McMaster University - Mechanical Engineering.

this. The average torque is shown in equation (5-1), while the current is simply assumed constant.

$$
T_{a v}=\frac{1}{2} \cdot \frac{L_{a}-L_{u}}{\beta_{r}} \cdot i^{2}
$$

where $L_{a}$ and $L_{u}$ are inductance at aligned and unaligned position.

At the same time, the copper loss is also proportional to the square of current, as shown in (5-2), where $R$ is the copper resistance of single phase.

$$
P_{\text {copper }}=R \cdot i^{2}
$$

It's very obvious that, the copper loss is almost proportional to the motor output torque. The copper loss calculation is following the steps shown in Fig. 5.2. Following the relationship and steps, the copper loss variation for one velocity cycle is obtained and shown in Fig. 5.3.

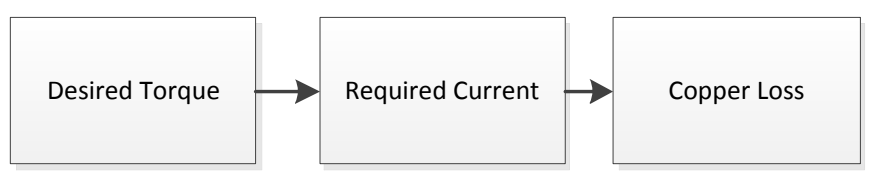

Fig. 5.2. Copper losses calculation for a typical velocity cycle.

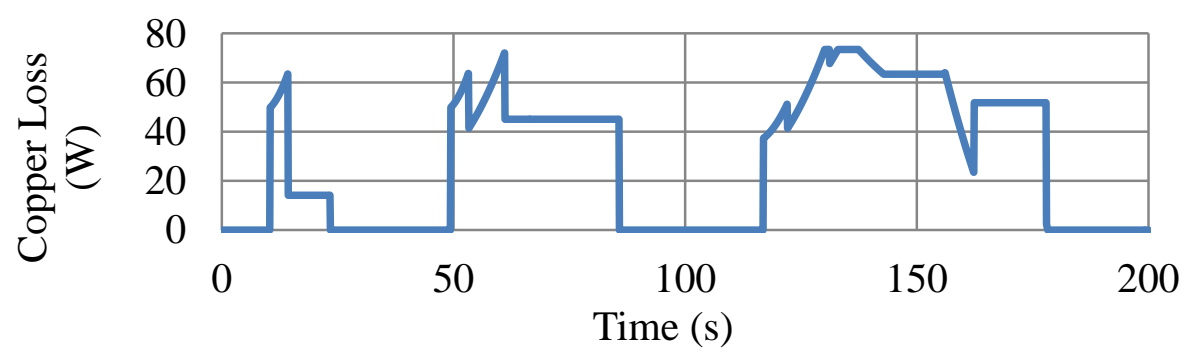

Fig. 5.3. Copper loss variation for one velocity cycle. 
M.A.Sc. Thesis - Jianing Lin; McMaster University - Mechanical Engineering.

\subsubsection{Iron Loss}

Iron losses in cores include eddy current loss $P_{e}$ and hysteresis loss $P_{h}$.It's assumed that the eddy loss is about $20 \%$ of the total iron loss, and the hysteresis loss is about $80 \%$ [59]. With the knowledge of the stator and rotor iron losses at rated condition, with speed of 500rpm, also as known,

$$
\begin{aligned}
& P_{e} \propto f^{2} \\
& P_{h} \propto f
\end{aligned}
$$

where $f$ is the flux variation frequency at rotor or stator core, which has a linear relationship with the rotation speed for a designed machine.

The iron losses are simply calculated in Fig. 5.4, with (a) is the iron losses on stator and (b) is on rotor. By putting the output power, copper losses and iron losses together as shown in Fig. 5.5, the efficiency variation for one velocity cycle is shown in Fig. 5.6. Thus, the average losses and efficiency is calculated for one velocity cycle, as shown in Table 5.3. It will be used as the heat source of a realistic thermal analysis. 
M.A.Sc. Thesis - Jianing Lin; McMaster University - Mechanical Engineering.

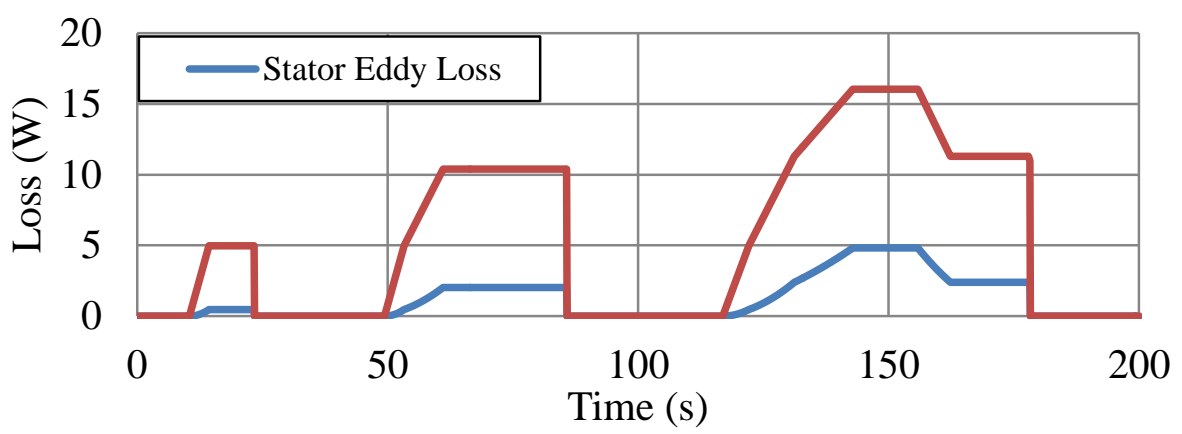

(a)

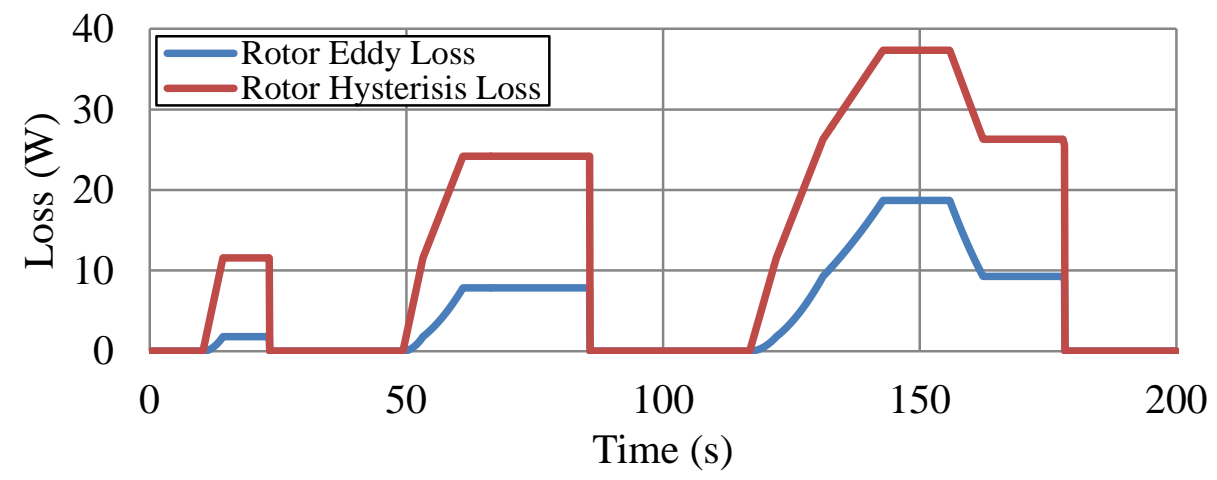

(b)

Fig. 5.4. Iron losses variation for one velocity cycle.

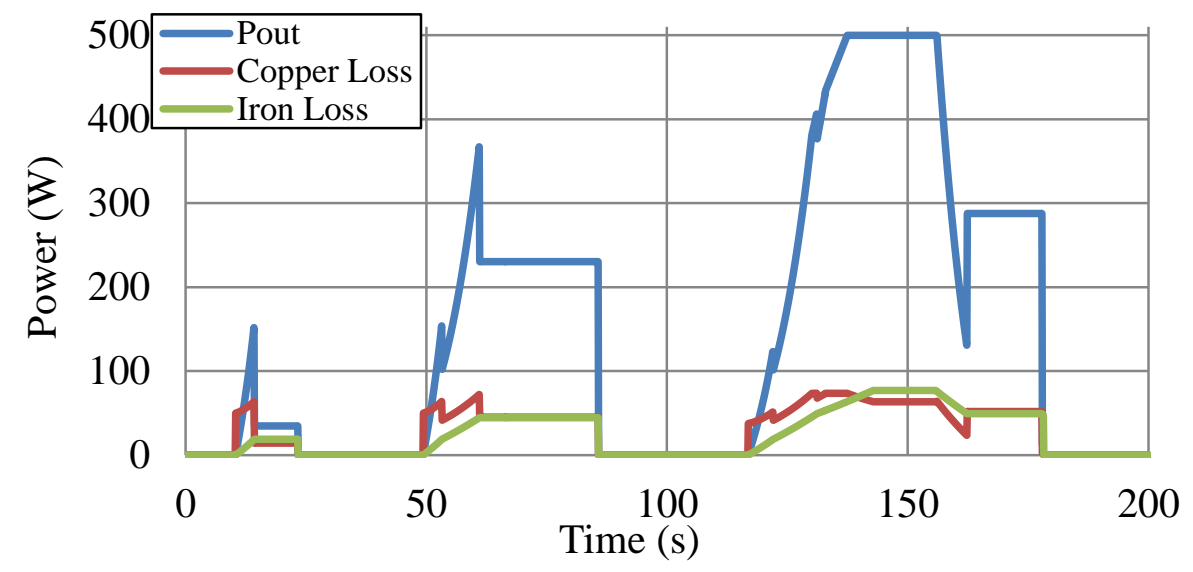

Fig. 5.5. Output power, copper and iron losses variation for one velocity cycle. 
M.A.Sc. Thesis - Jianing Lin; McMaster University - Mechanical Engineering.

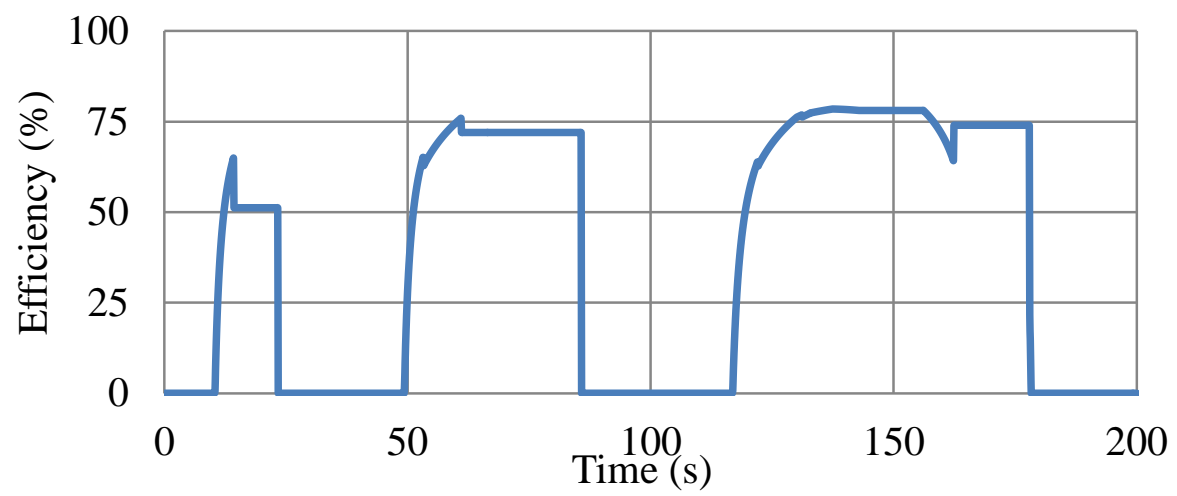

Fig. 5.6. Efficiency variation for one velocity cycle.

Table 5.3. Average losses for one velocity cycle.

\begin{tabular}{l|l|l|l}
\hline $\begin{array}{l}\text { Average Stator } \\
\text { Iron Loss (W) }\end{array}$ & $\begin{array}{l}\text { Average Rotor } \\
\text { Iron Loss (W) }\end{array}$ & $\begin{array}{l}\text { Average Copper } \\
\text { Loss (W) }\end{array}$ & $\begin{array}{l}\text { Efficiency for the } \\
\text { whole cycle (\%) }\end{array}$ \\
\hline 6.72 & 17.54 & 28.0 & 74 \\
\hline
\end{tabular}

\subsection{Transient Thermal Analysis}

The loss calculated above is the heat source for the temperature variation analysis. Then the boundary conditions should be set correctly before the thermal FE analysis, for example, the heat transfer coefficient between different parts and between part and air, the initial temperature as well as the contact thermal resistant between different parts [60].

A constant heat transfer coefficient is specified for the entire temperature range [61]. Since there is a special air gap between the rotor and stator, the heat transfer coefficient of the gap between stator core and rotor core is calculated with an empirical equation in (5-5).

$$
h_{1}=\frac{6.6}{10^{5}} \frac{v r^{0.67}}{\lg ^{0.33}}
$$

where $v r$ is the rotor rotation speed in $\mathrm{cm} / \mathrm{sec}$ and $\mathrm{lg}$ is the air gap width in $\mathrm{mm}$. 
M.A.Sc. Thesis - Jianing Lin; McMaster University - Mechanical Engineering.

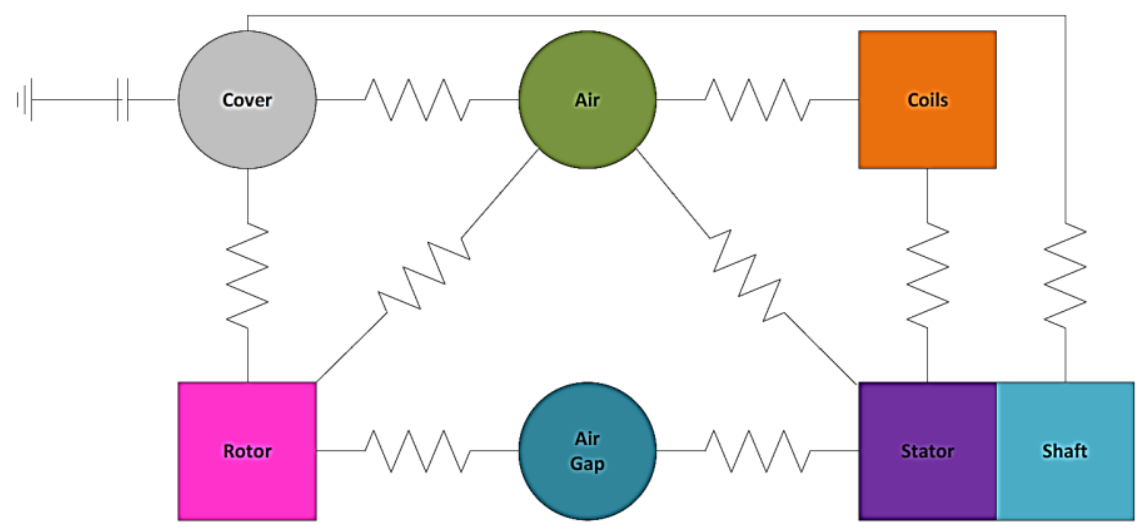

Fig. 5.7. Thermal connection between different parts.

Heat transfer coefficient between parts and air is set $15 \mathrm{~W} / \mathrm{m}^{2} \cdot$ Celsius. The thermal resistance caused by the insulation between the coil and stator, between rotor the cover and between shaft and cover is simulated using the contact thermal resistant condition. The value is finally chosen as $0.15 \mathrm{~W} / \mathrm{m} \cdot$ Celsius for the coil and stator, $0.027 \mathrm{~W} / \mathrm{m} \cdot$ Celsius for the others. Initial temperature is set to $20{ }^{\circ}$ Celsius as a standard room temperature. Thermal capability of the cover is set to be $100 \mathrm{~J} /{ }^{\circ} \mathrm{C}$. And the heat connections between different parts are shown in Fig.5.7.

\subsection{Results and Discussion}

Temperature Variation of different parts of the motor is then calculated by analysis the effect of loss for each element in JMAG.

Temperature Variation of different parts at rated operation condition with winding AWG 11 is shown in Fig.5.8, and the temptation distribution is shown in Fig.5.9. It is noticed that the coils have the highest temperature, which is caused by the copper loss they 
M.A.Sc. Thesis - Jianing Lin; McMaster University - Mechanical Engineering.

produce, as it's harder to eliminate this heat out than an external stator structure. At the time of 0.43 Hours, the temperature of coils starts to exceed $180^{\circ} \mathrm{C}$ which means unsafe for the motor's insulation thermo-stabilization requirement, thus extra cooling is needed to avoid this. However, this will absolutely increase the mechanical design complexity. Most important, it will increase the design cost.

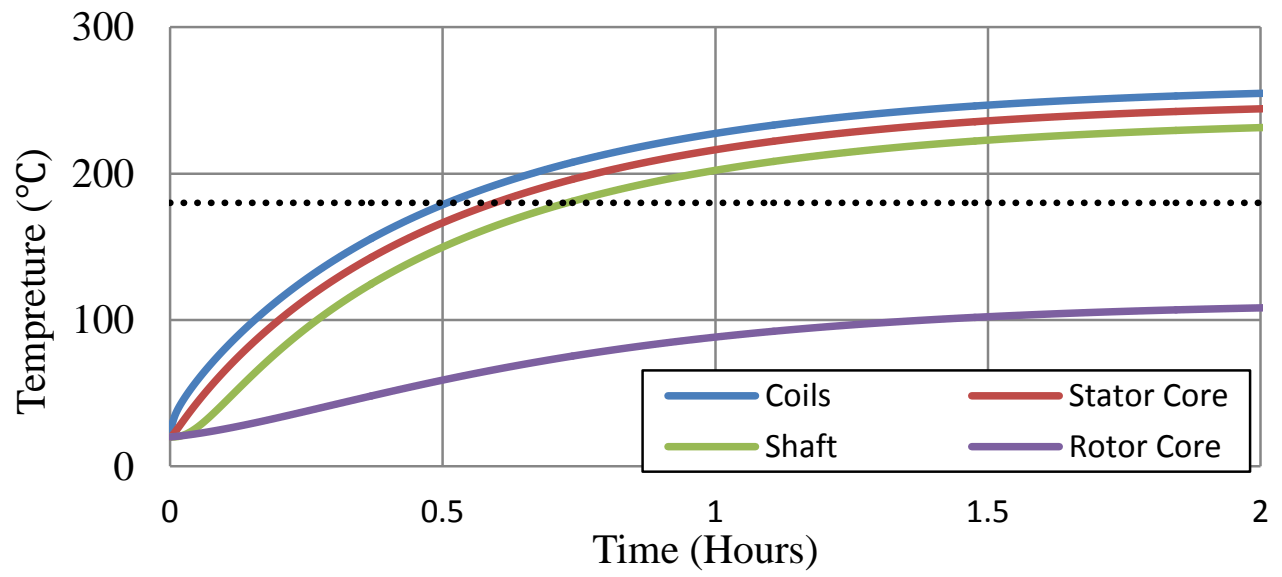

Fig. 5.8. Temperature variation at rated operation condition.
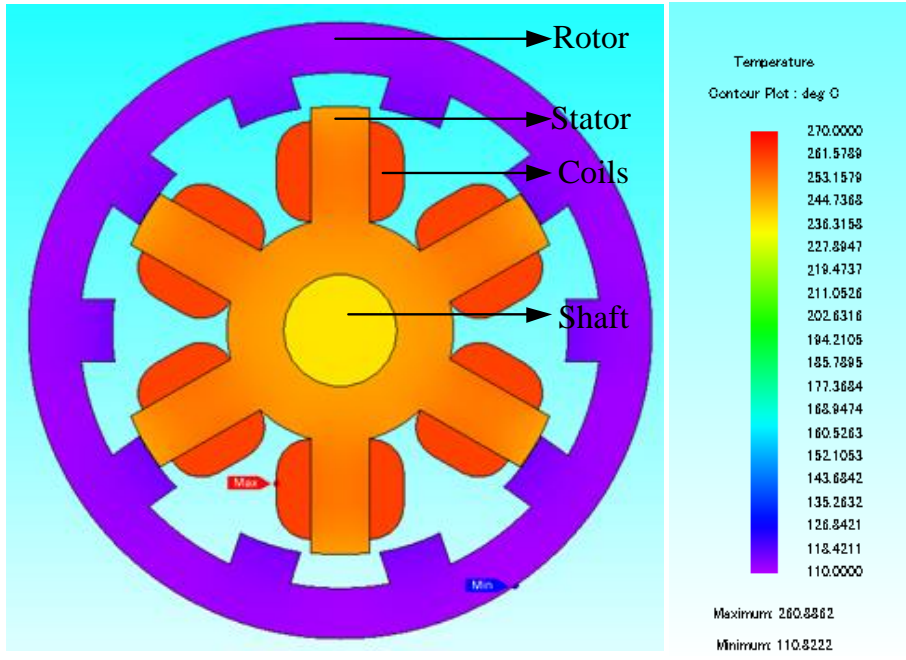

Fig. 5.9. Temperature distribution at rated operation condition. 
M.A.Sc. Thesis - Jianing Lin; McMaster University - Mechanical Engineering.

Thus, AWG 8 is recommended to decrease the copper losses and reduce the temperature increasing. Its temperature variation for different parts changes as shown in Fig.5.10. As shown, the temperature becomes stable at the time of 1.66 Hours. Till now, the heat generated by the motor equals to the radiating. A balance has been reached. The stable temperature of the coil is only $143^{\circ} \mathrm{C}$ lower than $180^{\circ} \mathrm{C}$. This meets the motor's insulation thermo-stabilization requirement.

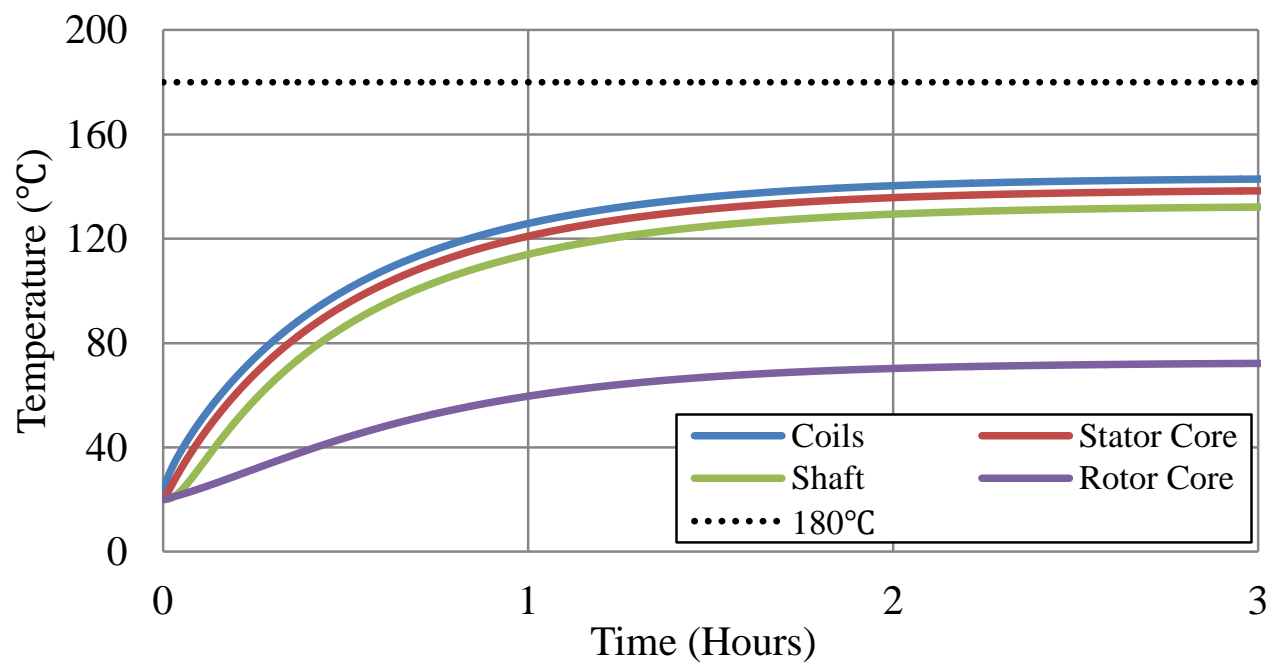

Fig. 5.10. Temperature variation with AWG\#8.

However, taking into account the velocity cycle, a realistic thermal analysis has been conducted instead of the temperature variation at rated motor operation. The typical urban cycle is already used to calculate the time-varying copper and iron losses. The required battery power for one velocity cycle is $0.0105 \mathrm{kWh}$. For a chosen $48 \mathrm{v} 10 \mathrm{Ah}$ Samsung LiMn battery with maximum current of $35 \mathrm{~A}$, it can provide $0.48 \mathrm{kWh}$ for each charge, supporting the motor for running about 3 hours. . The temperature variation caused by the calculated loss above for the 3 hours is expressed in Fig. 5.11. It shows that the 
M.A.Sc. Thesis - Jianing Lin; McMaster University - Mechanical Engineering.

temperature is only $80^{\circ} \mathrm{C}$ for the coils, which is safe enough. Considering the hand winding for the prototype motor, AWG11 is still used.

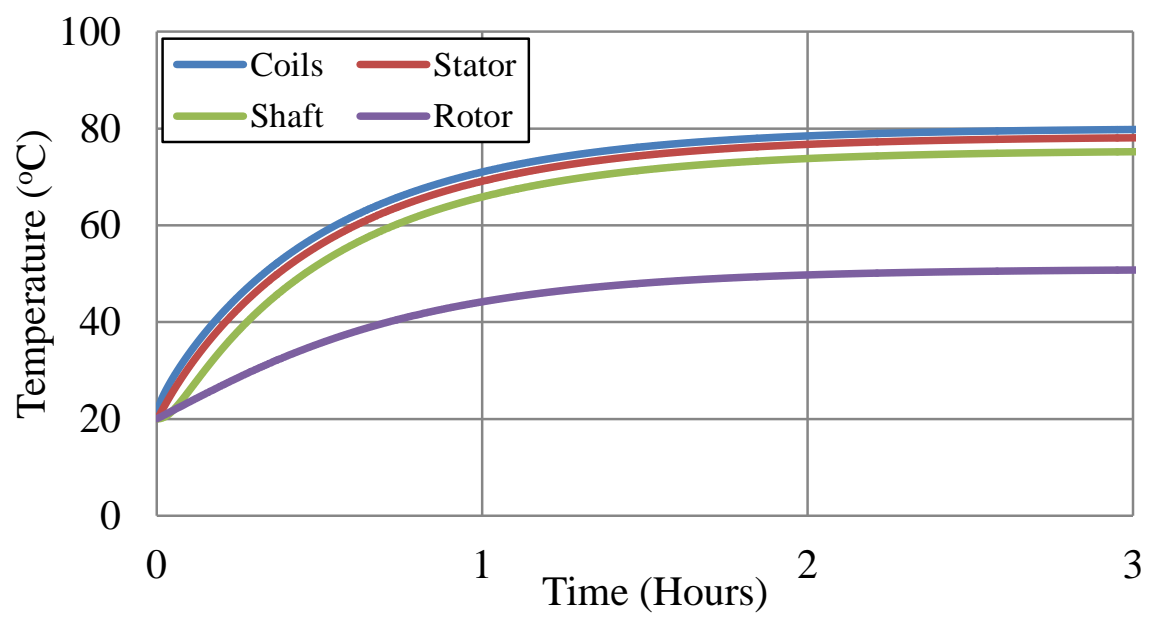

Fig.5.11. Temperature variation from average loss of velocity cycle.

\subsection{Conclusions}

For the internal stator, thermal safety verification is more important for the machine, especially with a current of 35A. Detail FEA is conducted for copper losses and iron losses calculation as well at the temperature variation and distribution. At the rated condition, conduction cross-section increase can be a good way for the temperature reduction. However, thinking about the realist condition, the average losses for the velocity cycle is more convincing for machine's thermal analysis. In this situation, it's proved that all the parts can be kept within a very safe temperature of $80^{\circ} \mathrm{C}$, when there reaches a balance between the heat generation and radiation. 
M.A.Sc. Thesis - Jianing Lin; McMaster University - Mechanical Engineering.

\section{Chapter 6 Prototype Motor Mechanical Realization}

To realizing the design and verify the analysis results, a test bench is set-up. The test system includes mainly two parts, one is the motor prototype, and the other is the power electronics, which include the converter board and the control box to drive the motor. Besides that, the power supply here is using a controllable DC power source for test purpose, which is better to control the current and voltage output than the $48 \mathrm{~V}$ battery, used on the E-bike.

For this external-rotor design, the hub motor structure is used, with the internal stator located on the static shaft and the external rotor on the rotational case. As discussed in Fig. 3.1, to realize the speed legal in Ontario, a 3:1 reduction gear box will be applied on this motor, whose sunny gear will be connected with the rotational case and spike located on the ring gear. However, for the experimental test consideration, the mechanical design is more considering test convenience. The expansion view of motor is shown in Fig.6.1.
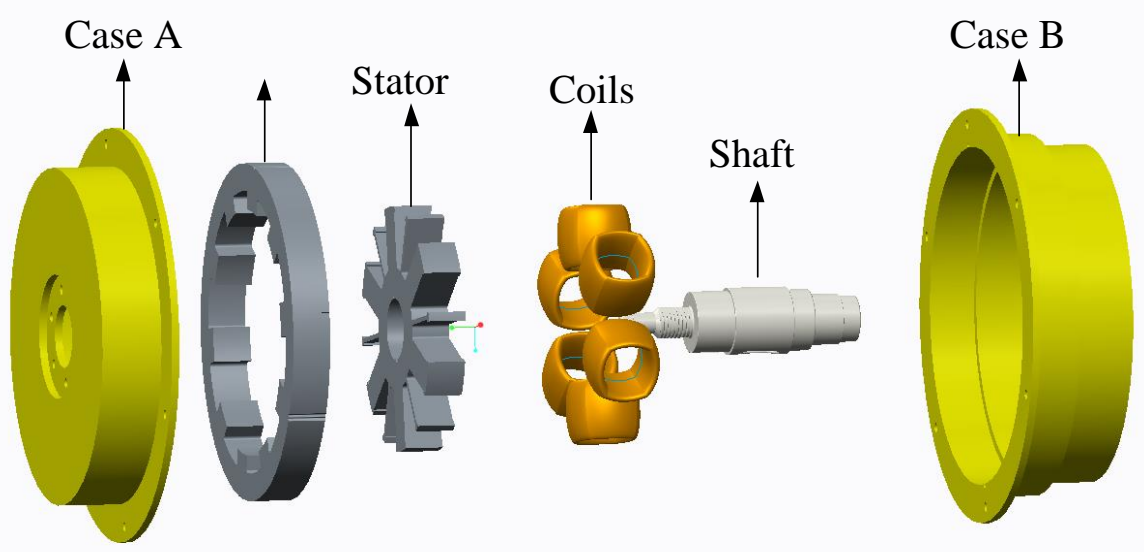

Fig. 6.1. Expansion view of the designed motor in 3D drawing. 
M.A.Sc. Thesis - Jianing Lin; McMaster University - Mechanical Engineering.

\subsection{Lamination Design}

Compared with the conventional SRM, the in-wheel design leads to an interior stator construction. Considering winding, the interior stator has the advantage of larger slot open, which makes the winding more easily. However, the larger slot open also introduce a big problem of instability of holding the windings in the slot during the operation. Considering this, wedges are used to locate the windings. To hold the wedges, tips on stator poles are needed.

As the stator pole length is long, this will introduce a very big distance between the two poles. One wedge is hard to hold, which also results in a big loss of the winding space, because the wedge is usually chosen to be hard enough, which is difficult to bend. It's clearly a straight line between the tips of two adjacent poles will waste a lot of winding space. Considering this, a narrow part is added in the middle of the two poles. The initial stator and the one with wedge tips are compared in Fig.6.2.

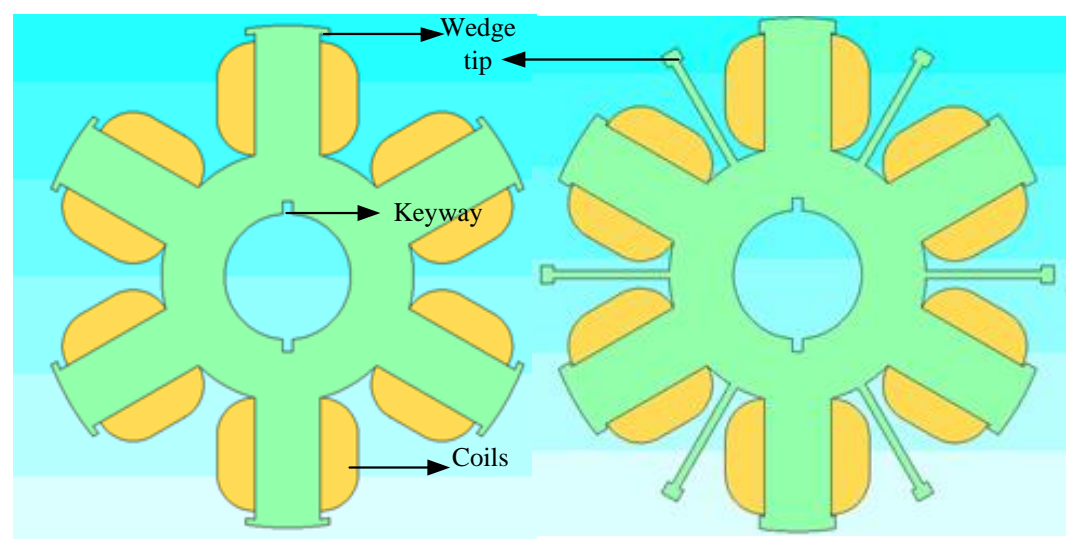

Fig.6.2. Comparison of the original stator and the design with wedge tips. 
M.A.Sc. Thesis - Jianing Lin; McMaster University - Mechanical Engineering.

However, the tips may influence the output performance of the motor. A detail analysis of choosing the geometry and dimensions of these tips are conducted in FEA. The analysis shows that the added tips will increase the inductance at unaligned position. And this will also reduce the difference of aligned inductance over unaligned inductance, which will lead to a reduction of the output average torque. However, it shows that the reduction is very slightly.

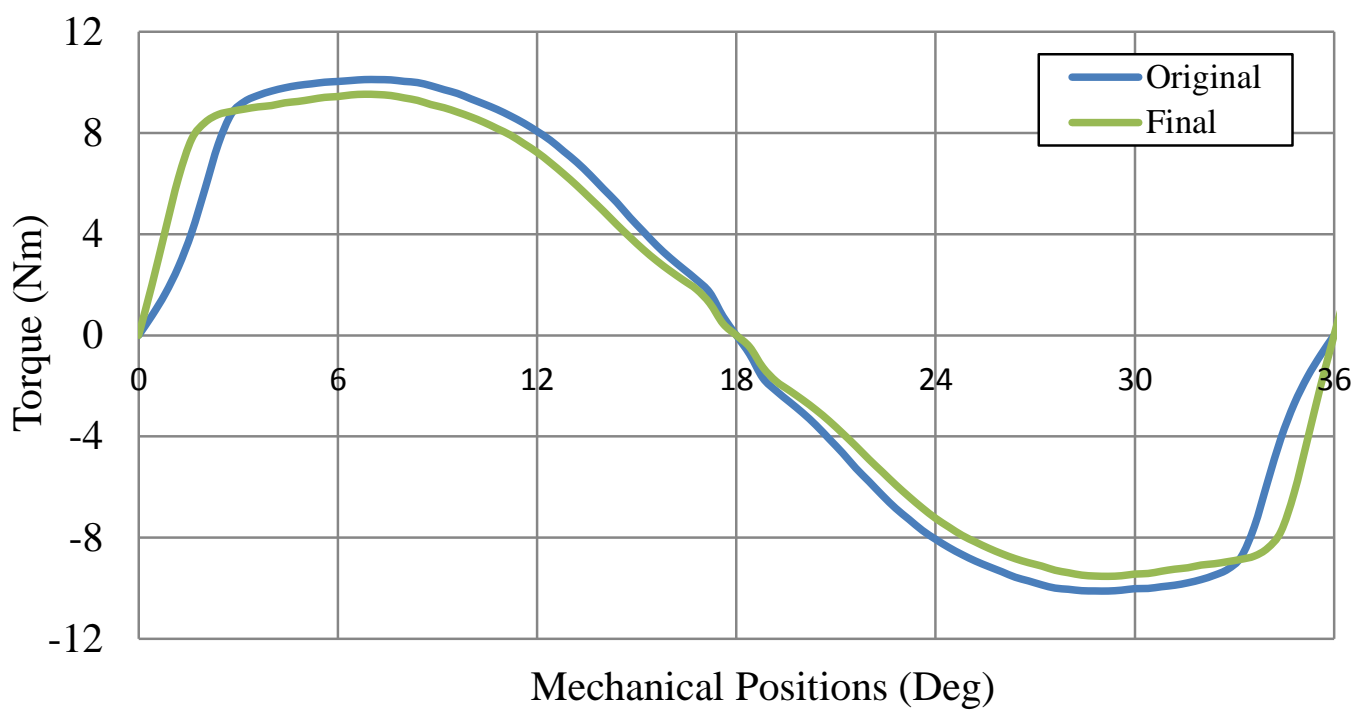

Fig. 6.3. Comparison of the original design, the final design with wedge tips.

Table.6.1. Comparison of the origin stator design and the final design with wedge tips.

\begin{tabular}{l|c|c|c}
\hline & Average Torque $(\mathrm{Nm})$ & Torque Ripple & Filling Factor \\
\hline Origin & 8.63 & $42.90 \%$ & 0.31 \\
\hline Final & 8.73 & $40.87 \%$ & 0.35 \\
\hline
\end{tabular}


M.A.Sc. Thesis - Jianing Lin; McMaster University - Mechanical Engineering.

What's more, the added tips also increase the possibility to reduce the torque ripples. As shown in the Fig.6.3, the new structure has the torque over $8 \mathrm{Nm}$ more flat. With a simply APC control, the average torque and torque ripple for these two structure have been compared in Table. 6.1. There is a little drop for the average torque, and slightly decrease of the torque ripple. The flux distributions at unaligned and aligned positions are also shown bin Fig.6.4. When compared with the flux distribution figures for the original design in chapter 3, they are almost the same. Thus, taking into consideration the advantage and disadvantage, this special design is significantly good for the manufactory process, but the performance changing is small enough to be accepted.

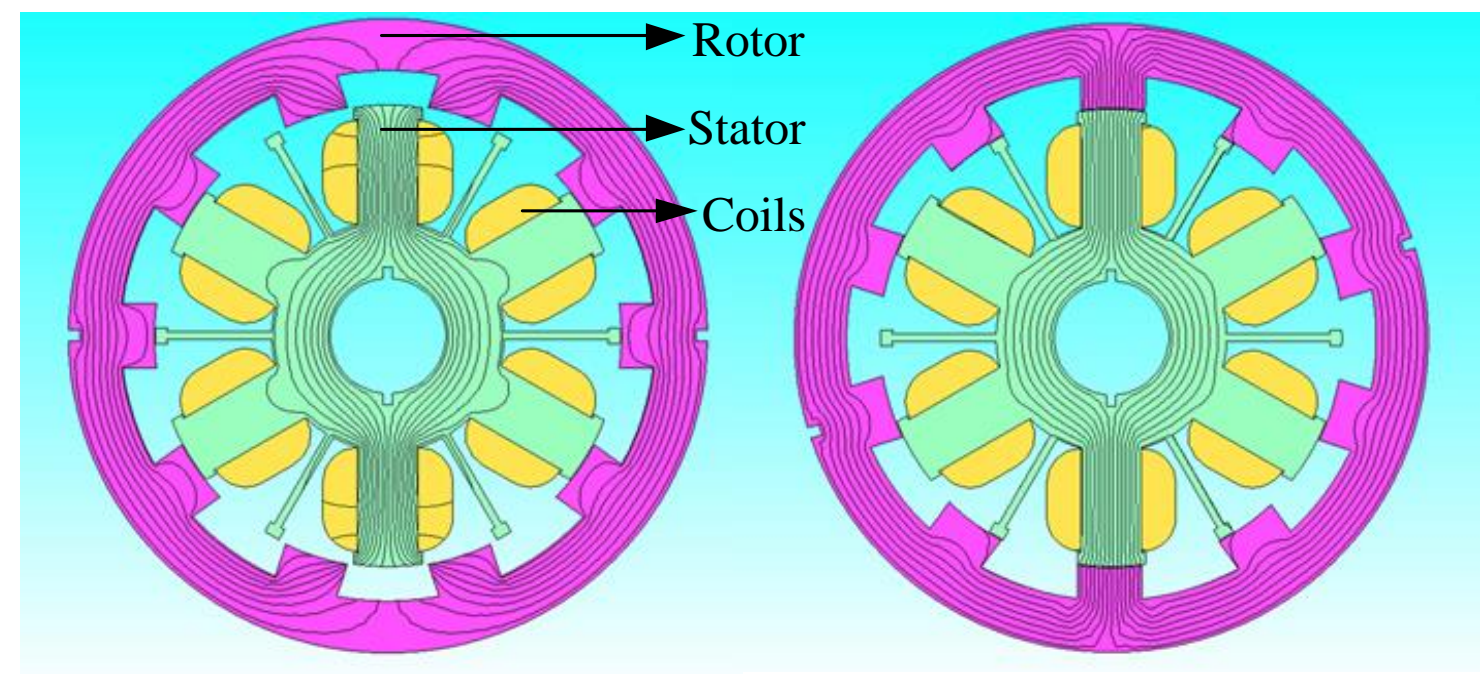

Fig. 6.4. Flux distribution on the final stator design with wedge tips. 
M.A.Sc. Thesis - Jianing Lin; McMaster University - Mechanical Engineering.

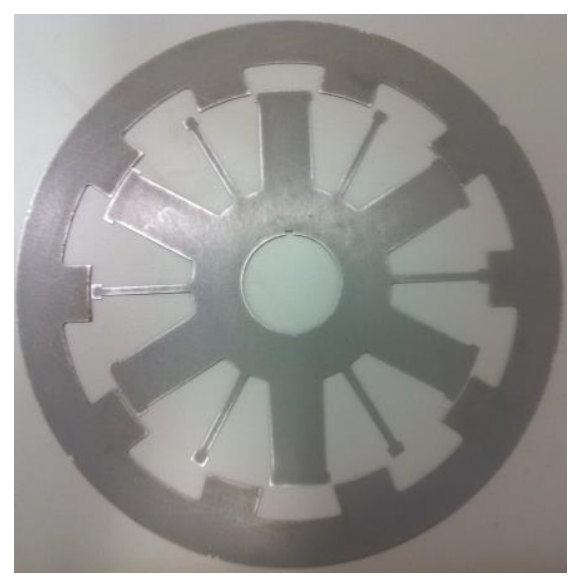

Fig.6.5. Lamination of the final stator and rotor.

Keyways are placed on those point where the flux distribution is small, to avoid big influence. Two keyways are designed on the hollow hole of the stator, and two on the outer side of the rotor yoke. The machined lamination for both stator and rotor is shown in Fig.6.5.

\subsection{Stator Mechanical Design}

\subsubsection{Shaft design}

The shaft is static in this design, which connected with the stator. So the first design requirement for the shaft is to hold the lamination stack. Keyway is used to avoid the lamination movement in circumferential redial. Screw thread design on the shaft with washer and nut is used to push the lamination together.

As the case is rotational together with the rotor, the winding is designed to come out from the static shaft, instead of the case. A hollow shaft construction is considered, enough 
M.A.Sc. Thesis - Jianing Lin; McMaster University - Mechanical Engineering.

space for the winding is needed. Wild steel is used as the material to provide enough strength. The 3D construction is shown in Fig. 6.6.

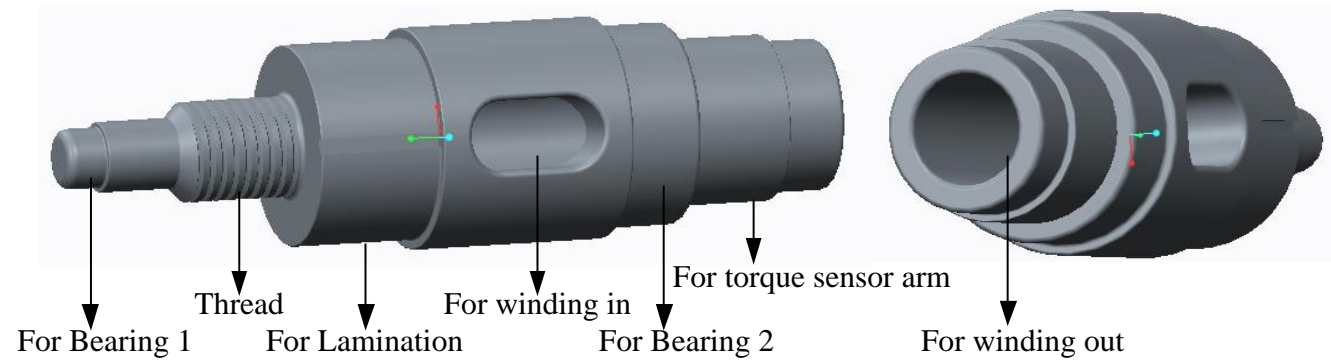

Fig.6.6. 3D shaft construction.

\subsubsection{Winding process}

The magnetic analysis chooses the AWG 11 for winding. However for the manufactory consideration, the AWG 24 is much easier for hand winding. The electric data is compared in Table 6.2.

Table.6.2. Parallel winding data [62].

\begin{tabular}{l|c|c|c}
\hline & $\begin{array}{c}\text { Cross-section } \\
\text { Area }(\mathrm{mm})\end{array}$ & $\begin{array}{c}\text { Maximum } \\
\text { Current }(\mathrm{A})\end{array}$ & $\begin{array}{c}\text { Resistance } \\
(\mathrm{mohm} / \mathrm{m})\end{array}$ \\
\hline AWG 11 & 4.17 & 39.3 & 4.132 \\
\hline AWG 24 & 0.20 & 1.93 & 84.22 \\
\hline $21 \times$ AWG 24 & 4.20 & 40.5 & 4.01 \\
\hline
\end{tabular}

21 parallel will also introduce an increasing of winding filling factor as the cross section area increases, but at the same time reduce the copper losses. As talked, the internal stator requires a wedge to hold the windings. To avoid winding damage, two insulation sheets are applied on both sides of the lamination stack. 
M.A.Sc. Thesis - Jianing Lin; McMaster University - Mechanical Engineering.

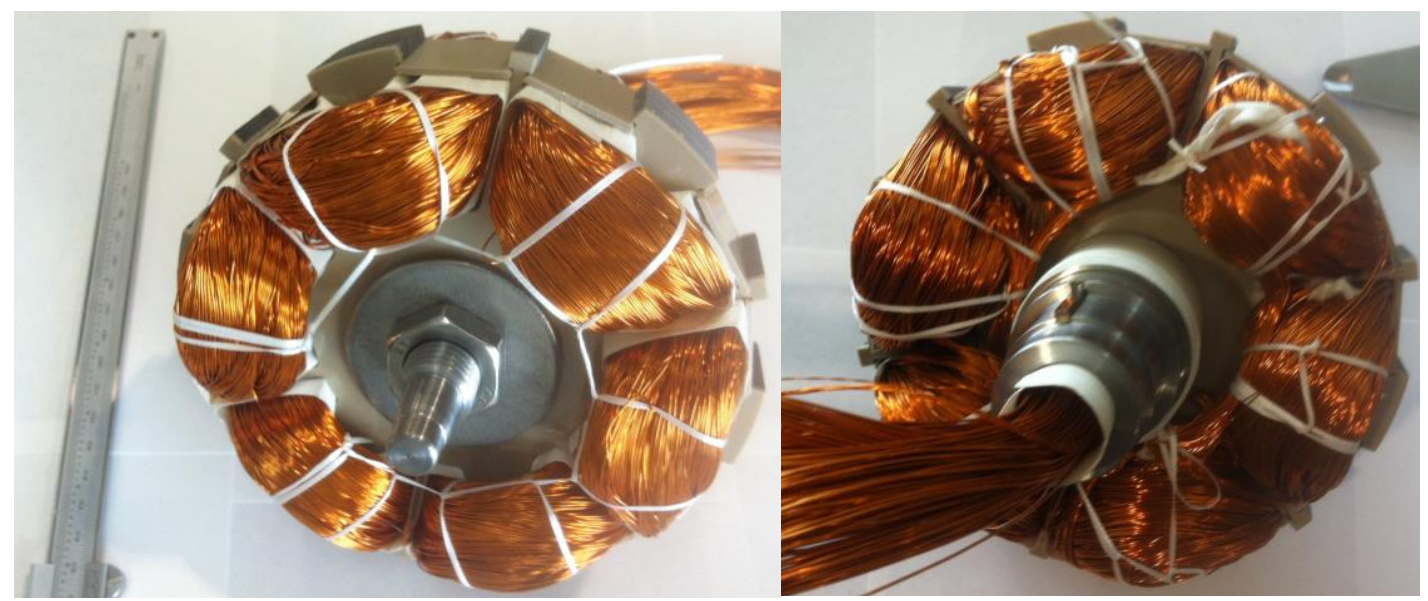

Fig. 6.7. Physical view of the stator on the shaft after winding.

After winding, insulation test between different phases, lamination stack and shaft is conducted to verify there is not short circuit. Vacuum pressure impregnation (VPI) is applied to make the connection between lamination tight enough. This is very important for the robust consideration of the stator and it can avoid additional vibration. The final structure of the designed stator is shown in Fig. 6.7.

\subsection{Rotor Mechanical Design}

The rotor lamination is welded together. Four welding points are chosen, which is on the outer diameter. As welding is possible to introduce electromagnetic and thermal effect on the stack, the welding points are choosing locations, where their flux linkages are small for the entire time. VPI is also applied on the rotor lamination.

For assembly the rotor stack and rotor case, shrink fit is used. Before shrink fit, there is the necessity to skim furnish the surface of the rotor lamination stack after welding. The rotor's outer diameter is $219.66 \mathrm{~mm}$ after skimming. For shrink fit, the mechanical 
M.A.Sc. Thesis - Jianing Lin; McMaster University - Mechanical Engineering.

parameters for these two materials are compared in Table 6.3, with electrical steel for rotor and aluminum for rotor case. Finally, S7h6 is chosen for the shrink fit.

Table. 6.3. Mechanical data comparison for the two shrink-fit parts [63], [64].

\begin{tabular}{l|c|c}
\hline & Young's modulus $\left(\times 10^{-5} \mathrm{MPa}\right)$ & Poisson's ratio \\
\hline Lamination & $1.95 \sim 2$ & $0.25 \sim 0.27$ \\
\hline Aluminum & 0.7 & 0.33 \\
\hline
\end{tabular}

\subsection{Other Issues}

To measure the position, an absolute encoder is chosen. As the shaft is statics in this project, an end part on the rotor case is developed to get the rotor's speed and position. There are totally 4 bearings used in this design, two for the case and shaft, and the other two for the brackets and shaft. By putting them together, the whole motor can be assembled; and its explosion view is shown in Fig. 6.9, with the physical view below. (a) is the physical rotor case with lamination and bearing 1 , (b) is the stator on the shaft with bearing 2, (c) is the other half of the rotor case and (d) is the designed rotational end part of the rotor case, which will connected with the encoder.

As the shaft is statics in this project, an arm is designed on it to measure reaction torque between the stator and rotor, which is also the output torque. The arm is getting the reaction torque to a force on the load cell. By reading the load cell is actually a strain gauge, and its electric resistance changes when the strain changes caused by the force. An amplification circuit gets this electric resistance information as a voltage output. The torque arm is $150 \mathrm{~mm}$. A DC machine is used as the load machine, which is the same 
M.A.Sc. Thesis - Jianing Lin; McMaster University - Mechanical Engineering.

function of the gravitational, air friction, and wheels friction forces on the E-bike. A pulley system is applied to connect these two machines together.

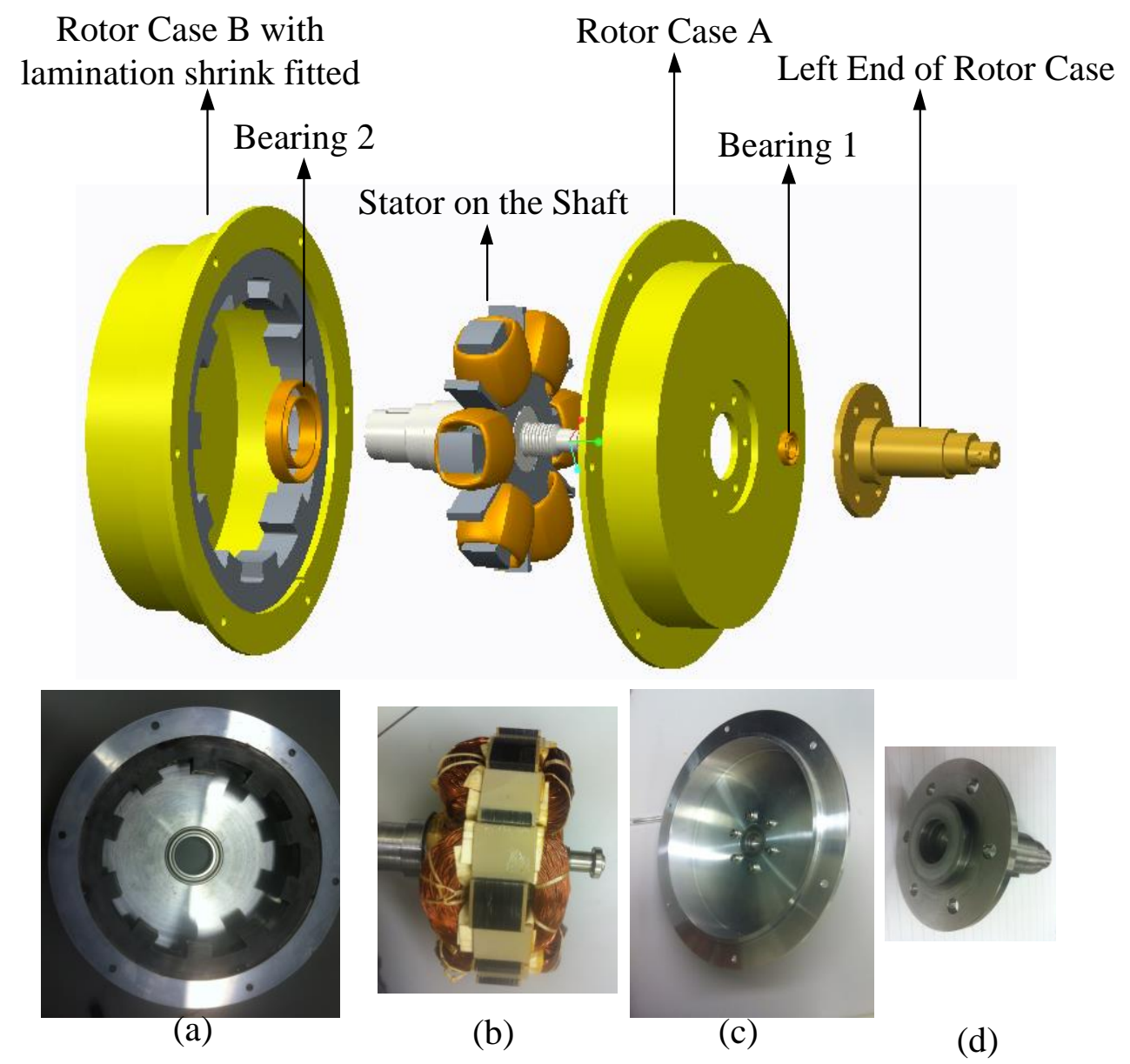

Fig. 6.8. 3D assembly view with physical parts below.

\subsection{Conclusions}

During the prototype machine manufactory, wedge tips are designed on the stator lamination, which can hold the winding in the slot. The rotor lamination is welded, skimmed, and shrink fitted in the cases. Using 11 parallel strands of the AWG 21 wire is 
M.A.Sc. Thesis - Jianing Lin; McMaster University - Mechanical Engineering.

easier for hand-winding, which is used instead of the original AWG 11 wire. Hollow shaft structure becomes the solution for getting the winding out from the machine, and load cell is used to measure the output average torque. At the same time, pulley system connected with a DC machine works as the load for the SRM. 
M.A.Sc. Thesis - Jianing Lin; McMaster University - Mechanical Engineering.

\section{Chapter 7 Experimental Results}

Structure parameters of the SRM are already listed in Table 3.4 and 3.5. However, during the prototype manufactory, some machine processing factors have to be considered, especially as a hand-made machine. As already talked in Chapter 6, wedge tips are added on the stator lamination to hold the winding, and the rotor is welded, skimmed and shrink fitted. Also, the winding is 11 parallel strands of AWG 21, which is easier for hand winding, but increases the copper losses than AWG 8. At the same time, as two insulation sheets are placed on the two sides of the stator lamination stack, the thickness of stator has to be reduced to $21.8 \mathrm{~mm}$ instead of $25 \mathrm{~mm}$. However, the rotor is still $25 \mathrm{~mm}$ thick, which is the initial design parameter. Taking all the issues into consideration, the prototype machine's structure parameters are listed in Table 7.1.

Table 7.1 Structure parameters.

\begin{tabular}{l|l}
\hline Number of stator poles & 6 \\
\hline Number of rotor poles & 10 \\
\hline Rotor outer diameter & $219.66 \mathrm{~mm}$ \\
\hline Stator bore diameter & $40 \mathrm{~mm}$ \\
\hline Air gap & $0.4 \mathrm{~mm}$ \\
\hline Length of rotor & $25 \mathrm{~mm}$ \\
\hline Length of stator & $21.8 \mathrm{~mm}$ \\
\hline Stator pole arc & $15^{\circ}$ \\
\hline Rotor pole arc & $16^{\circ}$ \\
\hline Turns per phase & 104 \\
\hline Wire size & 11 parallel of AWG 21 \\
\hline Core material & M15 steel \\
\hline
\end{tabular}


M.A.Sc. Thesis - Jianing Lin; McMaster University - Mechanical Engineering.

At the same time, there are some assumptions made during the FE analysis. As 3D model is not practicable, especially when the dimension of end winding is not available and its modeling accuracy is low, 2D model is used to represent the machine. However, this is only applied on the normal electric machines, which has much larger dimension in longitudinal direction than in radial direction. As for the designed 6/10 SRM, it's flatter than conventional SRM. Its end winding may have larger influence on its performance.

In addition, the lamination material of the prototype may not be exactly the same as that from the database applied on the analyzed model. Also considering the lamination factor, which respects the tightness between lamination, it will also introduce a flux drop especially at the aligned position, because the space between the lamination will lead to a higher saturation. At the same time, there will be test error during the experiment. They will all lead to a difference between the analysis and test results.

\subsection{Static Tests}

Torque and flux-linkage profiles are of the most important characteristics describing a designed machine. The 2D FEA results are already presented in Chapter 3 . To verify the analysis, the prototype's torque and flux linkage profiles are necessary to be checked before the dynamic test. The static test bench is shown in Fig. 7.1.

As seen in Fig. 7.1, the stator is locked by the load cell arm which located on the shaft. The rotor on the case is hold by a protractor, which is also called rotor position holder. By incrementally rotate its dial, the relative position of the stator and rotor can be accurately achieved. For the stator, as the connection between the shaft and bracket is a 
M.A.Sc. Thesis - Jianing Lin; McMaster University - Mechanical Engineering.

bearing, the reaction torque between the rotor and stator are all placed on the load cell.

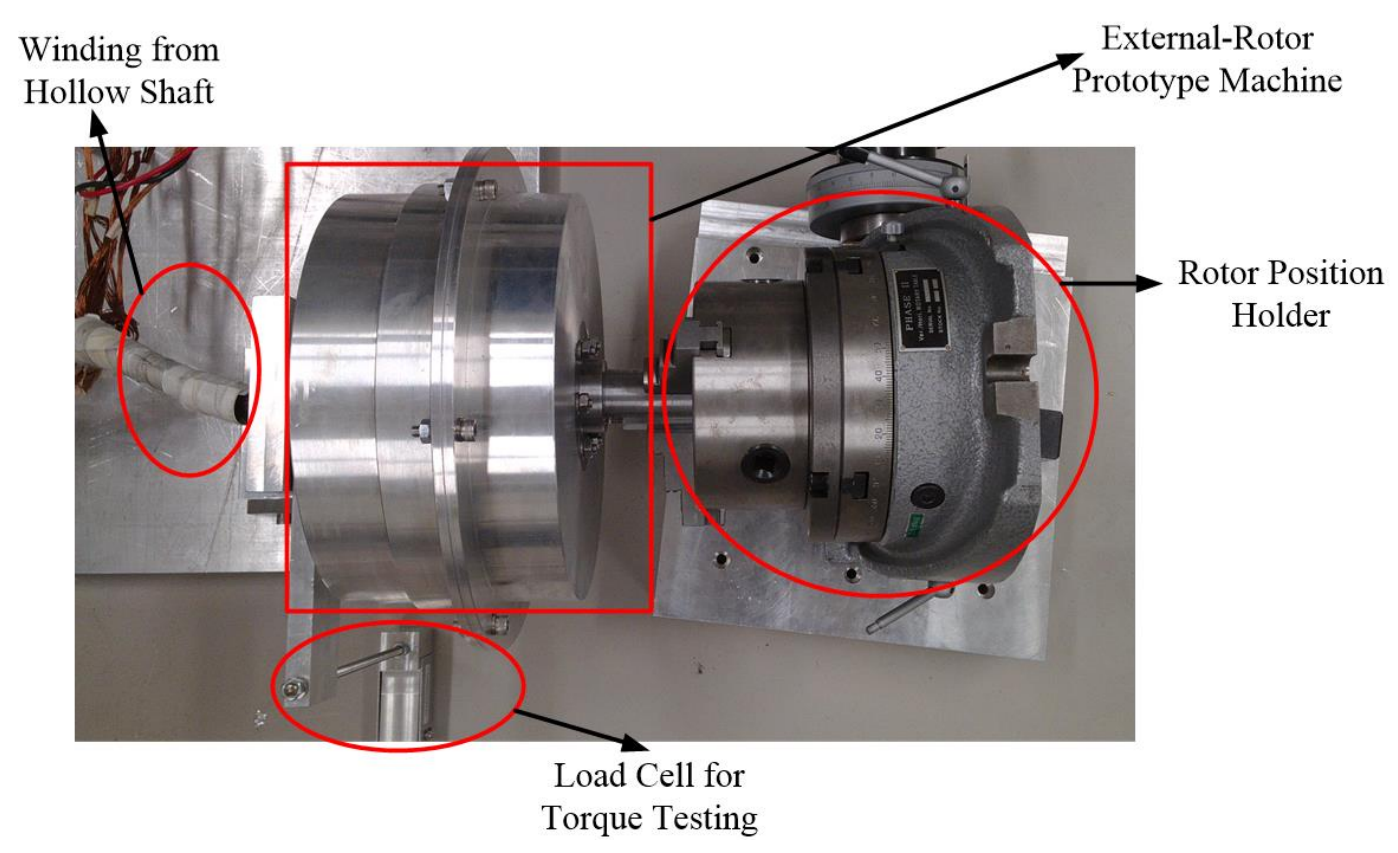

Fig. 7.1. Statics testing bench setup

For the static test, the torque and flux-linkage are measured with different currents at different position. The current range is from $0 \mathrm{~A}$ to $36 \mathrm{~A}$, with an increment step of $6 \mathrm{~A}$. The mechanical cycle of the $6 / 10 \mathrm{SRM}$ is 36 degrees. Since there is exact symmetry for the 36 degrees, only 18 degree is checked for the relative position between the stator and rotor. The aligned and unaligned position is picked by checking the output force, which should be both zero. However to improve the accuracy, the aligned position is chosen the one whose inductance is maximum and the unaligned is with the minimum inductance.

As one of the possible manufactory error for the SRM prototype is eccentric between the stator and rotor. The flux linkage profiles of two aligned positions are checked in Fig.7.2 to verify the concentricity. As it shows, the two flux linkage curves match with 
M.A.Sc. Thesis - Jianing Lin; McMaster University - Mechanical Engineering.

each other very well. That is to say, the inductance at different aligned positions are the same, which mean the air gaps are constant at aligned position. The flux linkage profiles for three phases are also measured in Fig.7.3. The consistency between them shows that the winding balance and mechanical symmetry can be guaranteed. This is considerably important to avoid vibration and noise during the operation.

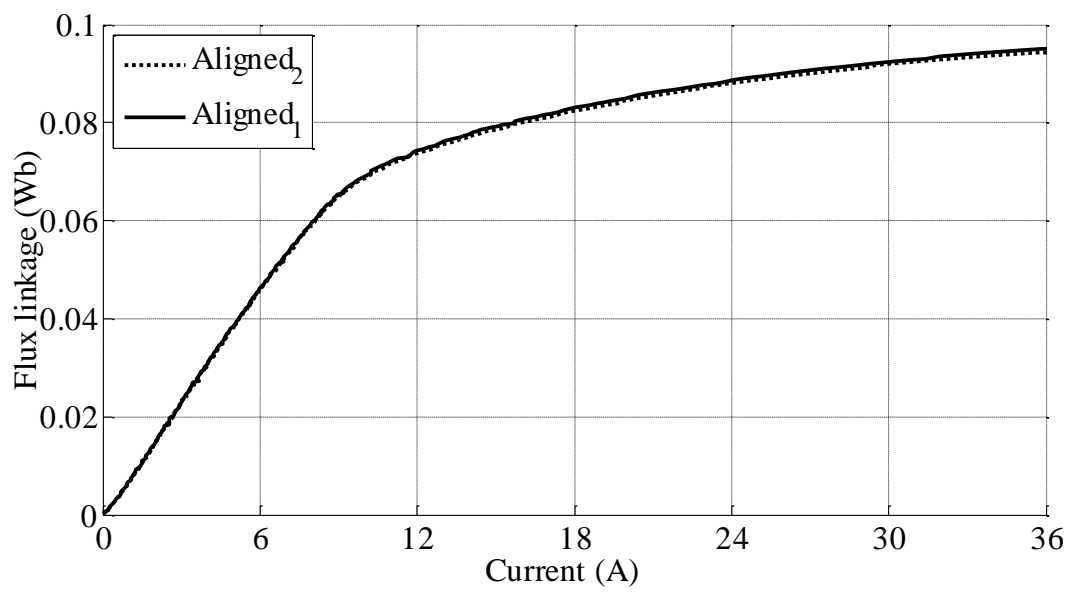

Fig. 7.2. Flux linkage curves at different aligned positions.

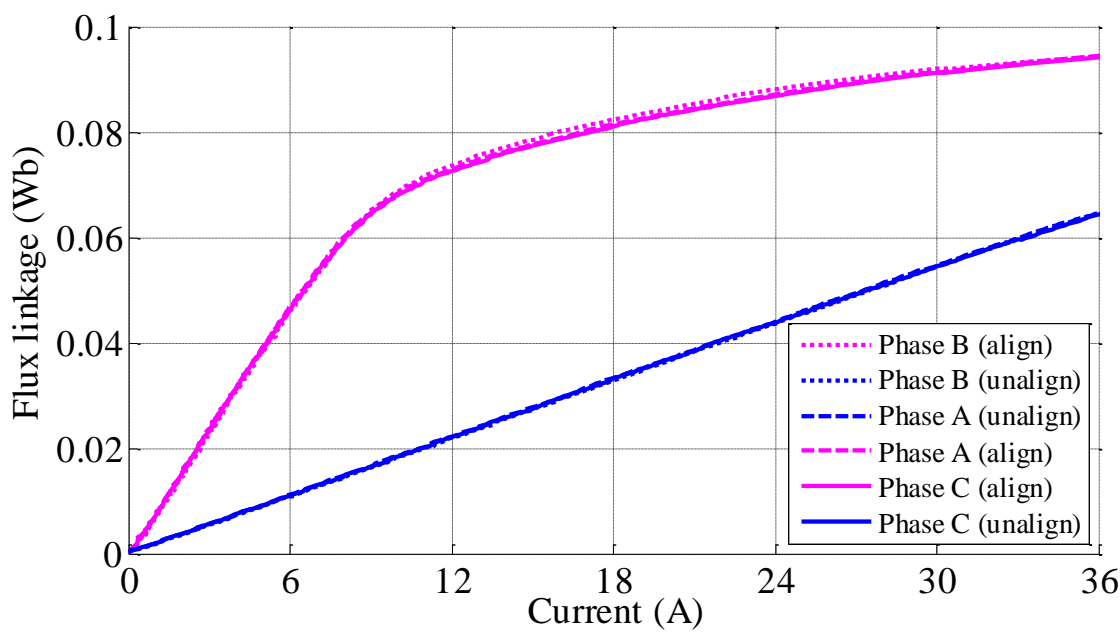

Fig. 7.3. Flux linkage curves comparison among three phases. 
M.A.Sc. Thesis - Jianing Lin; McMaster University - Mechanical Engineering.

After the verification of concentricity and phase balance, the flux linkage and torque profiles at static condition are compared with the $2 \mathrm{D}$ results in chapter 3 , which is an ideal model, without considering the end winding or the manufactory issues, for example, the inconsistence of the rotor and stator length.

The flux-linkage comparison is shown in Fig. 7.4, and the torque profile in Fig.7.5. As for the flux-linkage profile, there is big differences between the 2D FEA and the test results. For the unaligned position, the test results show a much higher slope than that from 2D FEA, which implies a higher inductance value at unaligned position. The reason can be smaller air gap at unaligned position, a better magnetic conducting material, or a bigger number of winding turns. Also, the wedge tip design introduces more flux leakage affect at unaligned position, which will lead to a smaller magnetic reluctance, and larger inductance.

As for the aligned position, especially for the saturated region, the flux-linkage value is lower for the test than the FEA results. It represents a lower inductance value. For material data difference, it is also check in FEA 2D, which shows very small difference with two totally different material data. But for the lamination factor, a looser contact may cause by the deeper saturated. A deeper saturation results in a lower inductance value. 
M.A.Sc. Thesis - Jianing Lin; McMaster University - Mechanical Engineering.

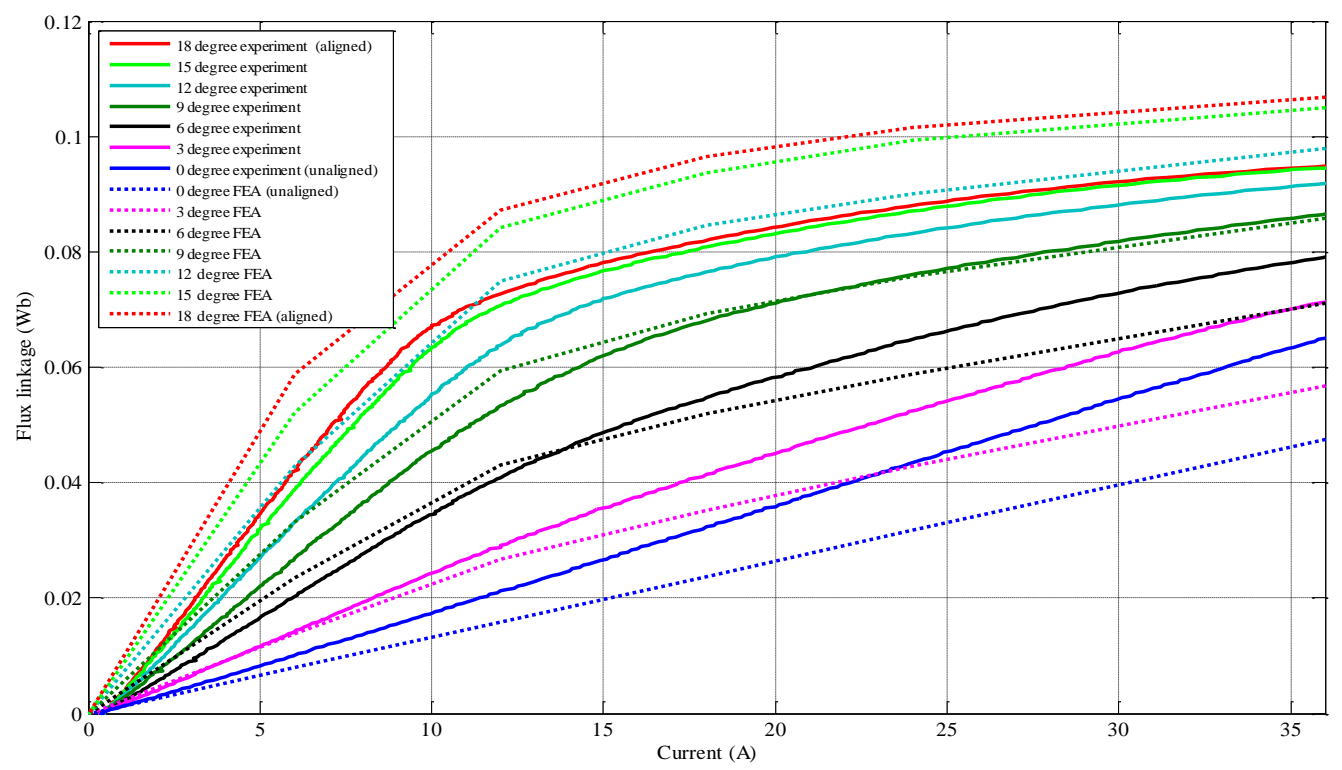

Fig. 7.4. Flux linkage profile comparison of the 2D FEA and experiment.

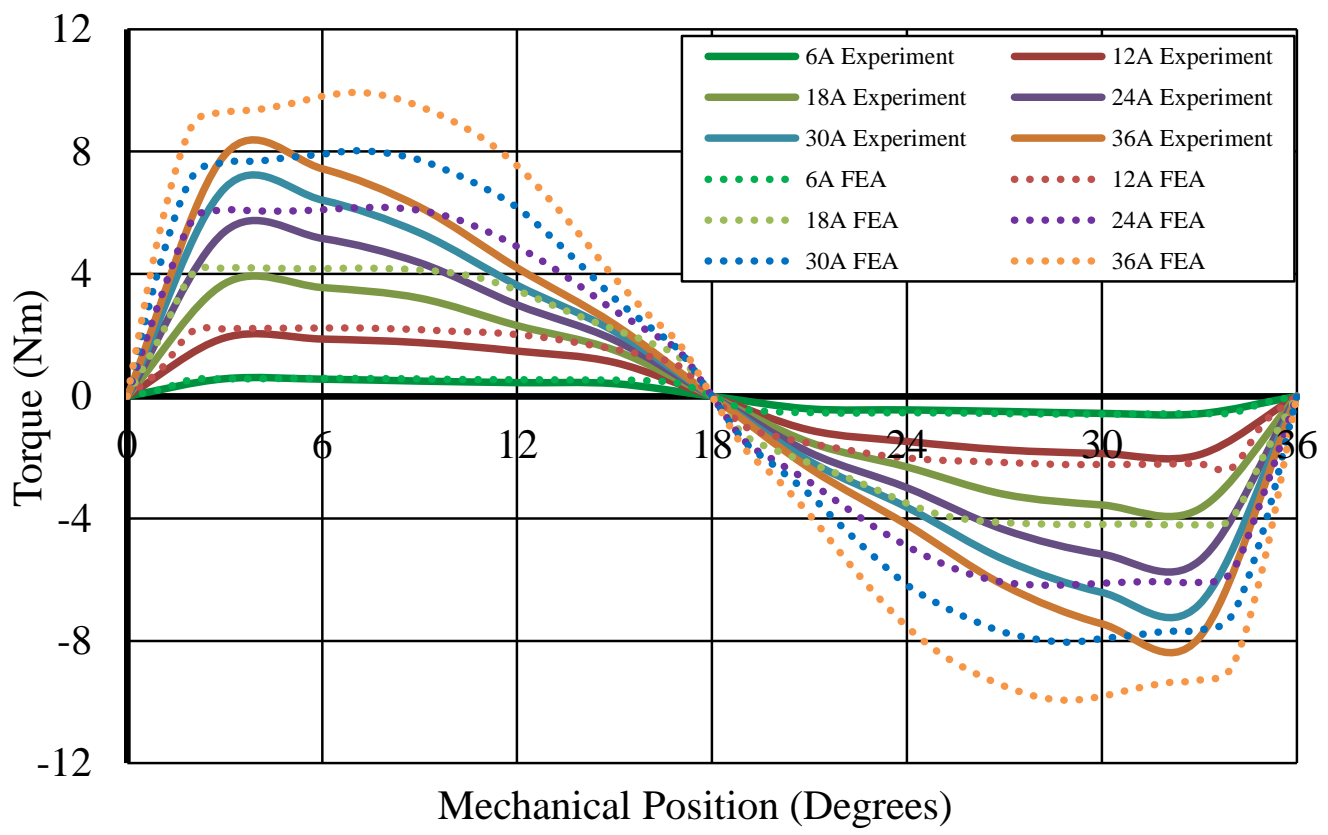

Fig. 7.5. Torque profile comparison between 2D FEA and testing.

To check the influence of wedge tips, the flux linkage with and without wedge tips in 
M.A.Sc. Thesis - Jianing Lin; McMaster University - Mechanical Engineering.

2D FEA are compared is shown in Fig. 7.6. It shows that adding the wedge tip will has bigger influence at unaligned position than aligned position. However, this difference with the test result is still very big.

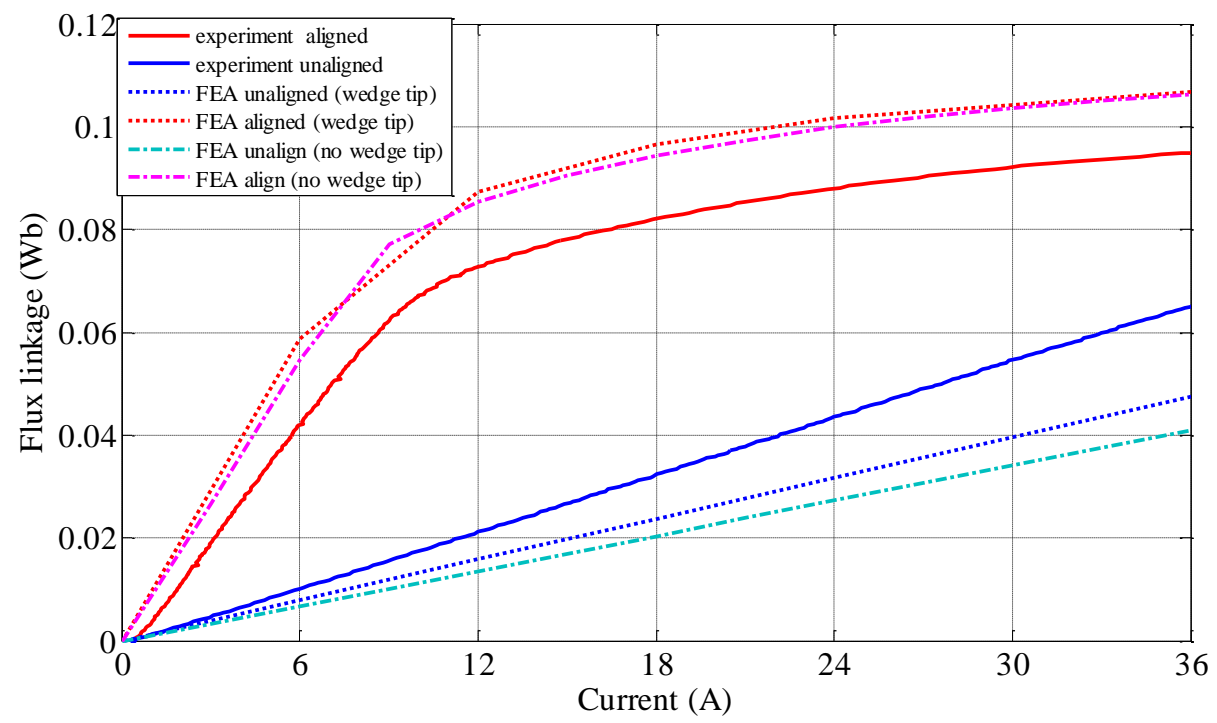

Fig. 7.6. Flux linkage comparison between FEA with/without wedge tip and testing.

To discuss the end winding influence, 3D FEA has to be studied with detail end winding modeling. As for 3D model, the inconsistence of the rotor and stator length, caused by the manufactory is also operable in FEA. Thus, the 3D model is simulated in FEA. It's compared with test results in Fig.7.7 and Fig.7.8. As shown, they match with each other much better. Considering the testing error, it's acceptable with the smaller difference between them.

From the new comparison, the conclusion is that the end winding's influence on this short stack machine is much bigger than the conventional ones. Its influence is mainly at the unaligned region. As the end winding can introduce more flux linkage with a certain 
M.A.Sc. Thesis - Jianing Lin; McMaster University - Mechanical Engineering.

current, so it will leads to a much bigger inductance. This is especially serious at unaligned position, which is not saturated. However, for the aligned position, which is saturated already, the flux-linkage will be limited to certain value, not increase as the ending winding added in. However, for the aligned position, the main reason is the change of stator thickness. For a same condition, a smaller thickness results in a lower capability of saturation. Thus, a lower saturated flux linkage value is shown in the test and $3 \mathrm{D}$ than the $2 \mathrm{D}$ result.

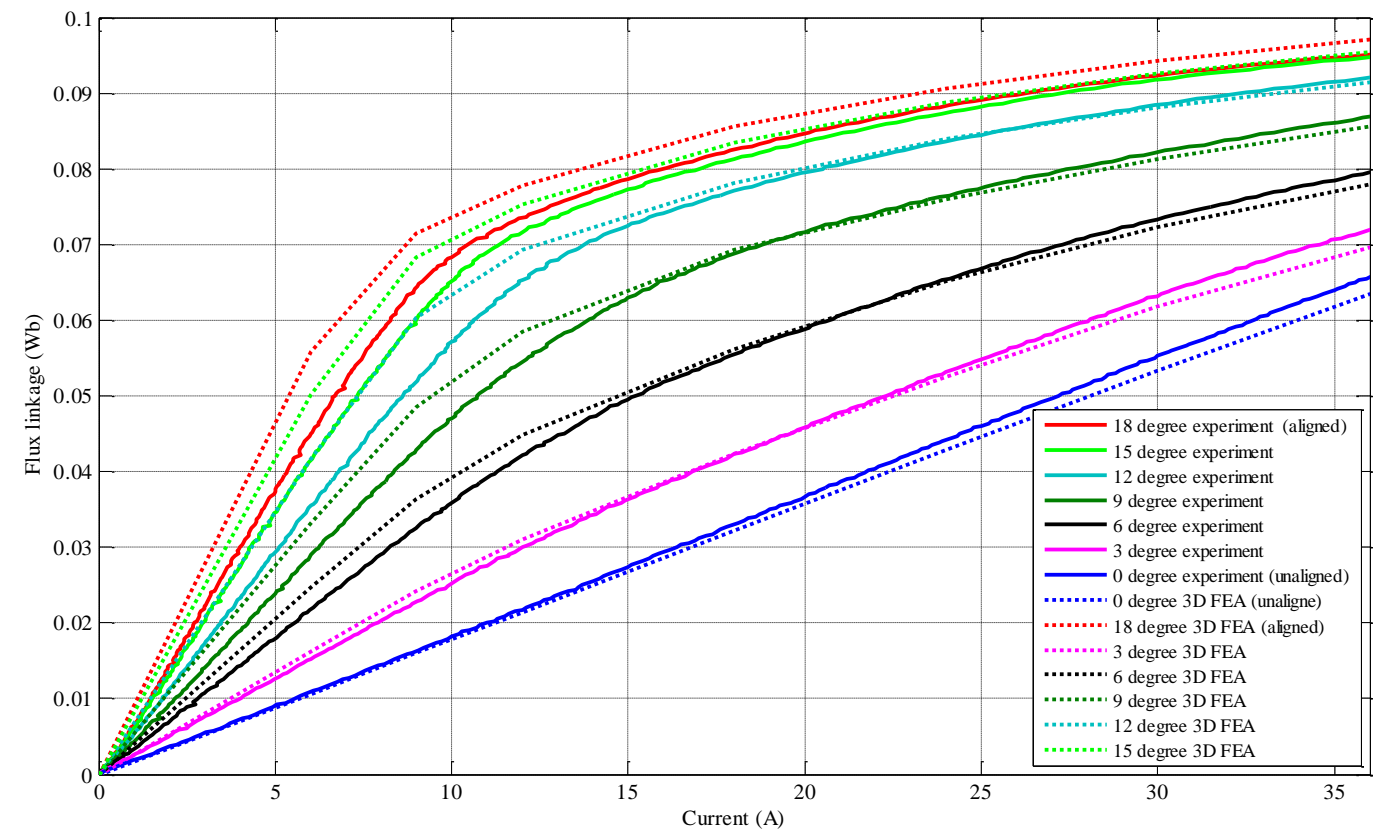

Fig. 7.7. Flux linkage comparison between 3D FEA and testing. 
M.A.Sc. Thesis - Jianing Lin; McMaster University - Mechanical Engineering.

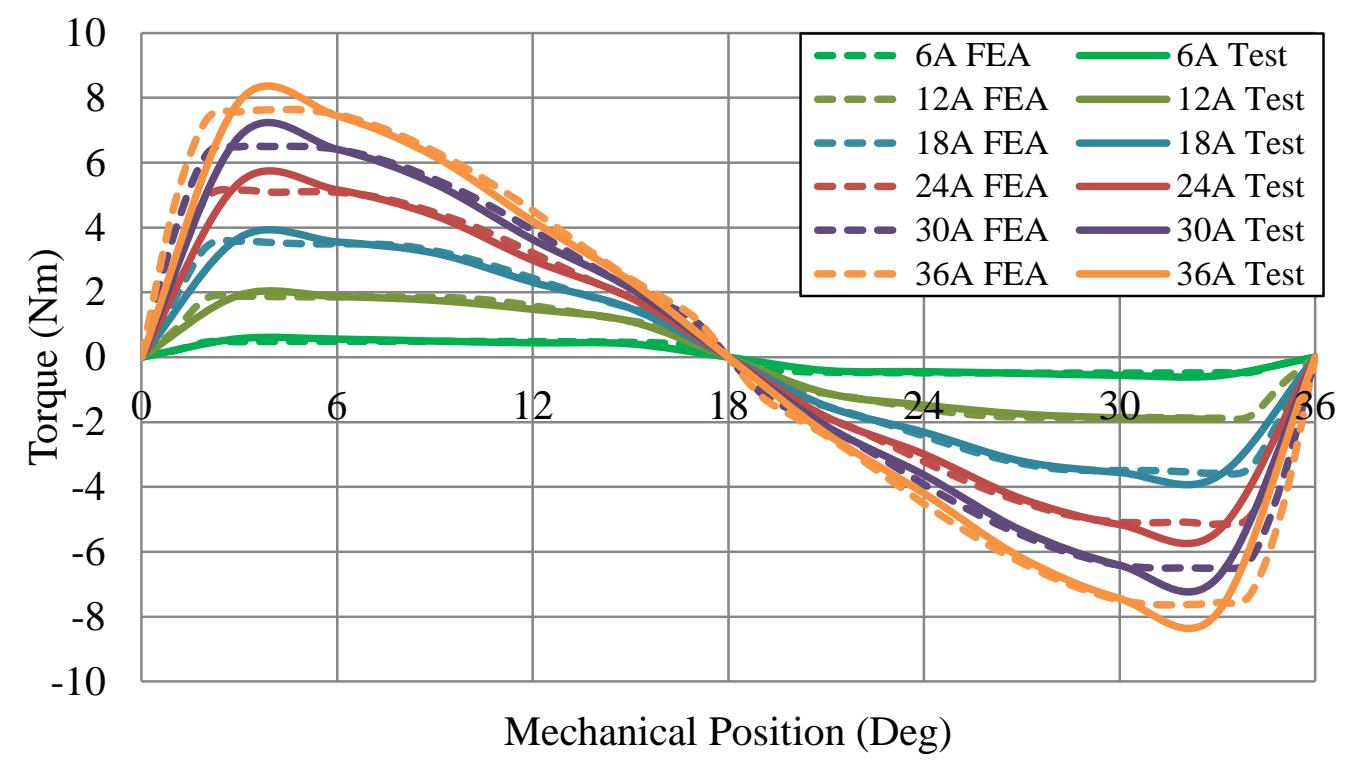

Fig. 7.8. Torque profile comparison between 3D FEA and testing.

As for the still existing difference for the unsaturated range at aligned position, the reason is explained by the test error. As talked in equation (2-1), the flux linkage can be calculated by integration of the input voltage minus the voltage drop at the electric resistance. This is used to calculate the flux linkage during test. By applying a voltage, there will introduce a transient increasing current from zero to rated value. By integrate the voltage at different current value, the flux-linkage is calculated. However, it only takes into condition the copper losses, but not the iron losses, which is resulted from the transient current change. An equivalent circuit in Fig.7.9 shows the influence of the iron losses for this testing. 
M.A.Sc. Thesis - Jianing Lin; McMaster University - Mechanical Engineering.

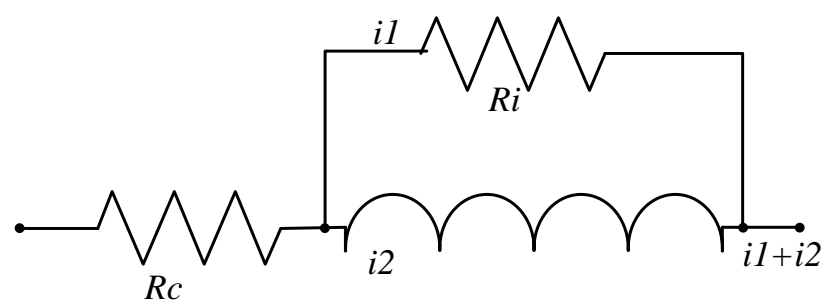

Fig. 7.9. Equivalent circuit for flux linkage calculation.

where $R_{c}$ is electric resistance of one phase, $R_{i}$ is the equivalent electric resistance for iron loss, $i_{1}$ is its equivalent current, and $i_{2}$ is the real current go through the coils. During the flux linkage calculation, the used current is actually $i_{1}+i_{2}$, which is bigger than the real current $i_{2}$. The influence is especially big for the smaller current range, as the equivalent iron loss current is of a bigger percentage.

\subsection{Dynamic Tests}

Different with static test, there are more issues introduced during the dynamic test. Fig. 7.10 shows the dynamic testing bench, which include:

(a) Prototype SRM with load system.

(b) Controllable DV voltage.

(c) Controller and converter system, fan cooling is introduced for this machine, as the rated current of $35 \mathrm{~A}$ is higher than normal applications.

(d) Oscilloscope for current and voltage recording, voltage meter for load cell output recording. 
M.A.Sc. Thesis - Jianing Lin; McMaster University - Mechanical Engineering.

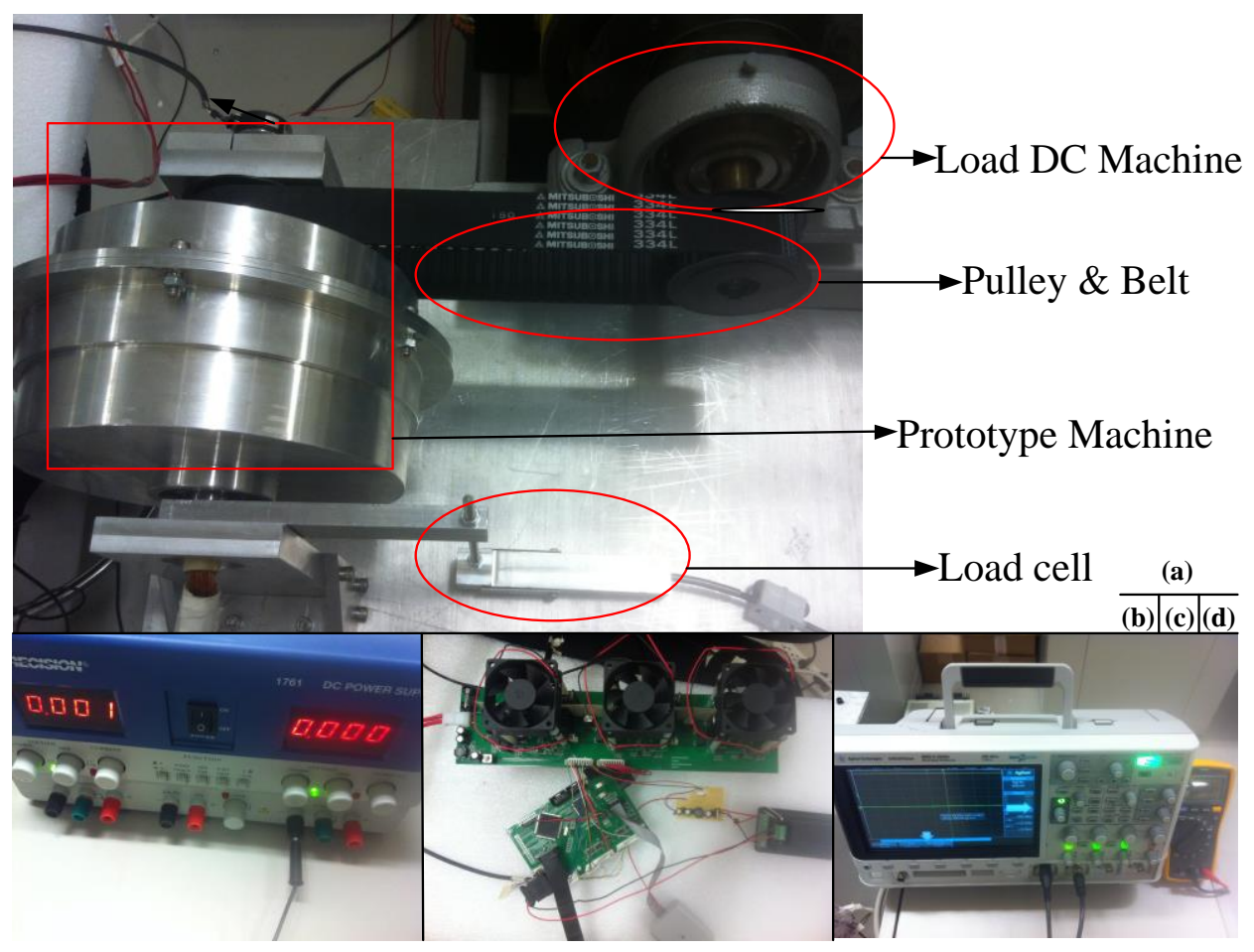

Fig. 7.10. Dynamics testing bench.

\subsubsection{No-load operation}

For the dynamic profile studying, no-load test is conducted. During the no-load test, the pulley and belt system in Fig.7.10 is disconnected. The testing is using the angular position control, with the turn-on angle of 0 degree and turn-off angle of 14 degree. The phase current is controlled. Its current and voltage at rated speed of 500rpm is record and shown in Fig.7.11. 
M.A.Sc. Thesis - Jianing Lin; McMaster University - Mechanical Engineering.
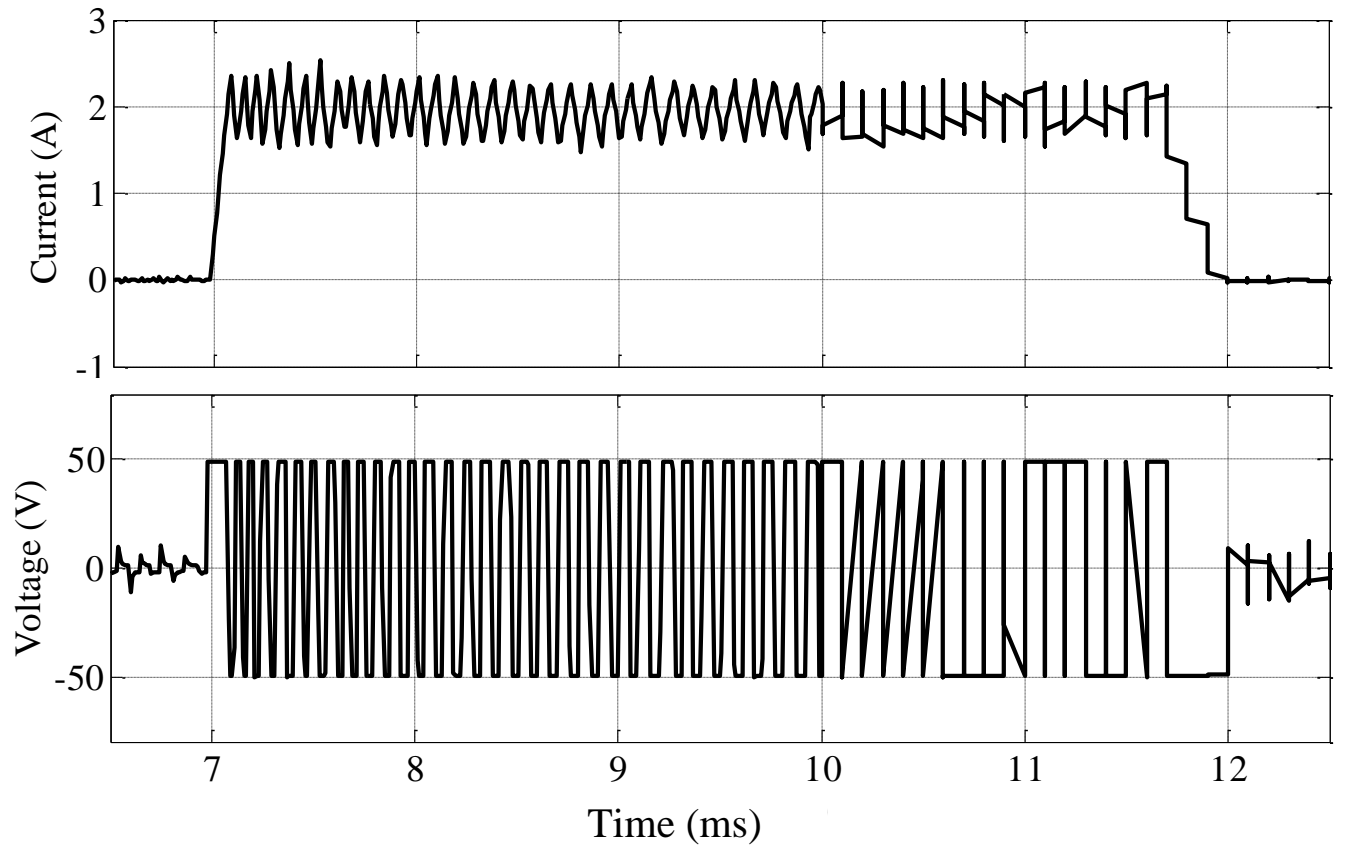

Fig. 7.11. Current and voltage at rated speed for no-load operation.

By summarizing the current and voltage in Fig. 7.11 together, the energy curve is calculated in Fig.7.12. As known from Chapter 2, the area integration of the closing triangle, whose $\mathrm{x}$-axis of current and $\mathrm{y}$-axis of flux linkage, will produce all the energy goes to the motor system. It includes all the mechanical output power, together with different losses. As the curve in Fig. 7.12 presents an operation status at a constant speed of 500rpm of the no-load test, there is no mechanical output power, which means all the energy goes to losses. It mainly includes the copper losses, iron losses and mechanical losses. However, as the current is only $2 \mathrm{~A}$, the copper losses and iron losses are both at a very low level. The energy of the no-load test can be considered the mechanical losses at the rated speed operation. The integration calculation demonstrates the mechanical losses are $3.3 \mathrm{~W}$. 
M.A.Sc. Thesis - Jianing Lin; McMaster University - Mechanical Engineering.

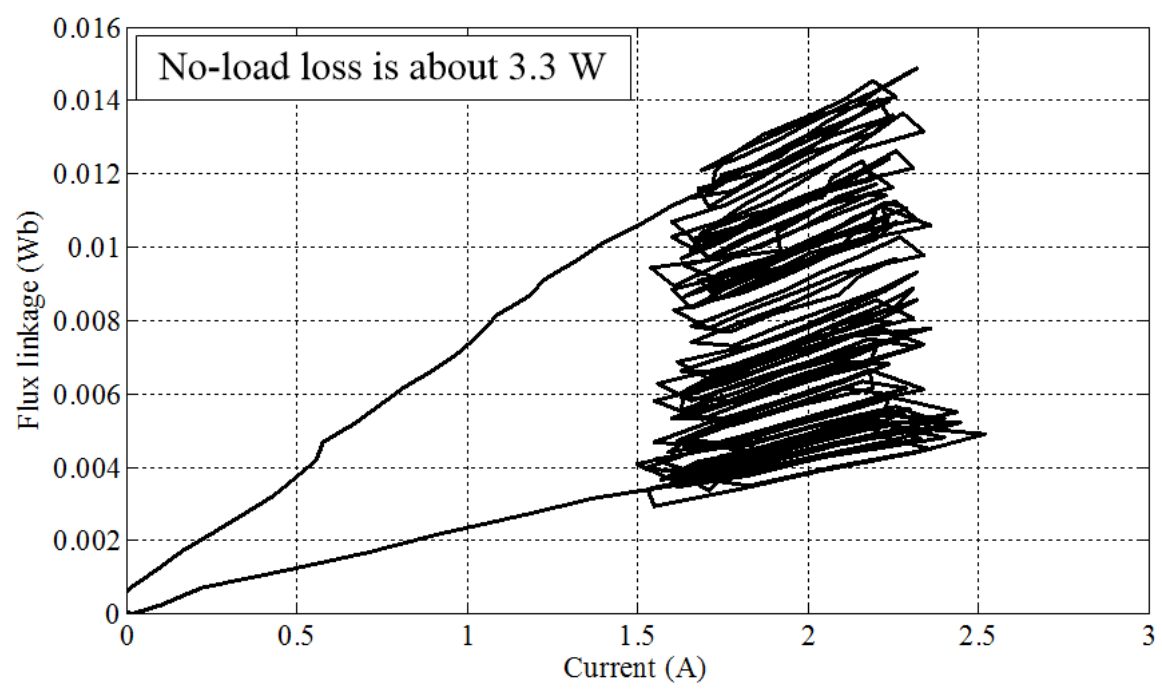

Fig. 7.12. Energy curve of no-load operation.

\subsubsection{Angular position control}

As discussed in chapter 4, turn-on and turn-off angles have very important effect on the machine's output performance, like output torque and torque ripple. In chapter 4, most work is focusing on the torque ripple reduction study, however, as the instantaneous torque is not measurable on the load cell at least now, the torque ripple reduction is not considered in this part, but will be studied in the future work. Only output torque magnitude is studied here.

First of all, the turn-off angle is studied. For a constant turn-on angle of 0 mechanical degree, which respect the unaligned position, the turn-off angle of 14, 15 and 16 degree is studied, as shown in Fig. 7.13. The energy curves shows include the output mechanical power, iron losses and copper losses. And the output mechanical energy is listed in Table 7.2. The current variation with the turn-on angle of 0 degree, but turn-off of 16 degree is shown in Fig. 7.14. 
M.A.Sc. Thesis - Jianing Lin; McMaster University - Mechanical Engineering.

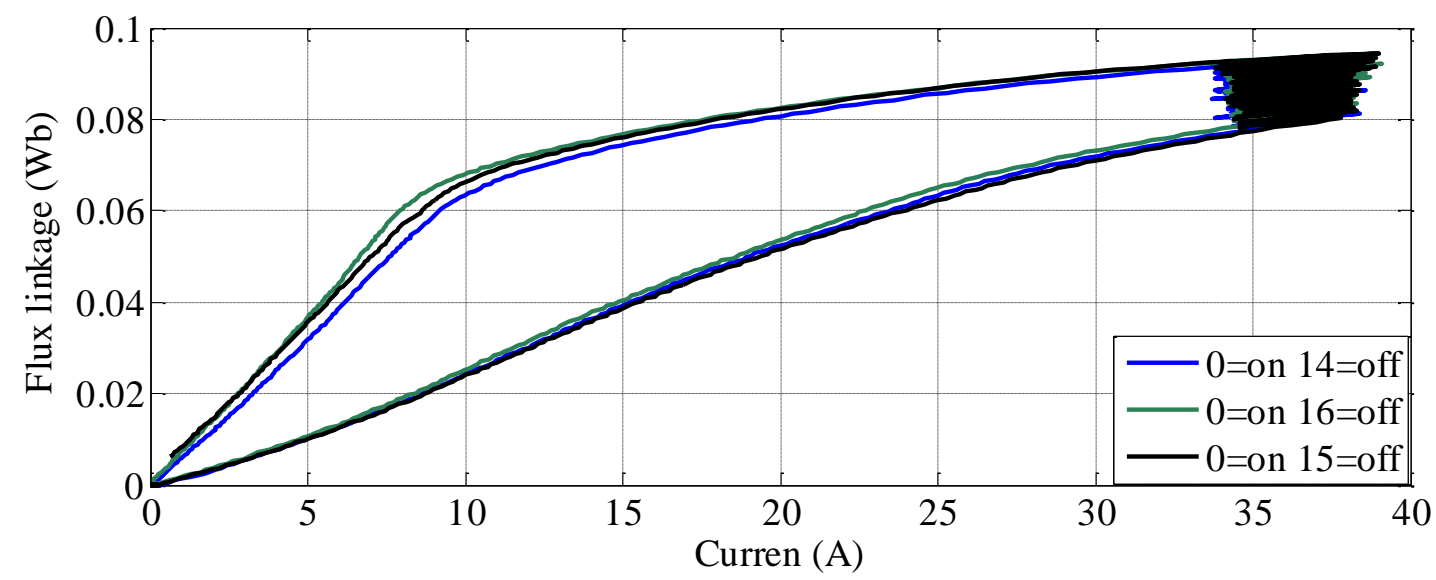

Fig. 7.13. Energy curve comparison for different turn-off but constant turn-on angles.

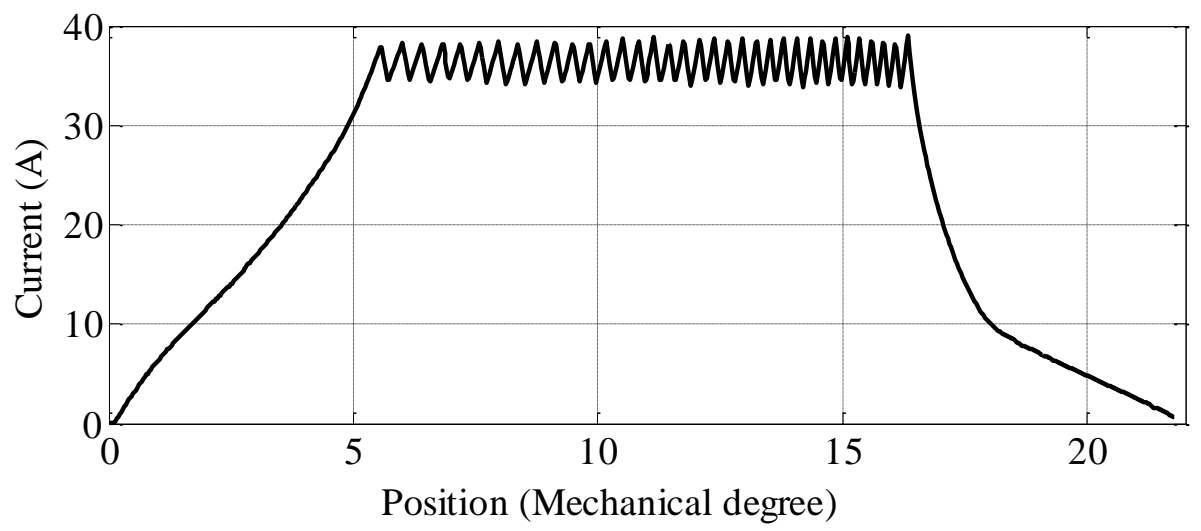

Fig. 7.14. Current profile with turn-on of 0 and turn-off of 16 degree.

Table 7.2. Output power comparison for different turn-off but constant turn-on angles

\begin{tabular}{|c|c|c|c|}
\hline Turn on (degree) & Turn off (degree) & Average torque $(\mathrm{Nm})$ & Output power $(\mathrm{W})$ \\
\hline 0 & 14 & 4.99 & 260.99 \\
\hline 0 & 15 & 5.07 & 265.07 \\
\hline 0 & 16 & 4.80 & 250.80 \\
\hline
\end{tabular}

As it shows that the turn off angle has very little influence on the machine's output performance. As known, the inductances at the turn-off positions are almost saturated 
M.A.Sc. Thesis - Jianing Lin; McMaster University - Mechanical Engineering.

inductance value, which is larger than that of the turn-on position. Thus, the variation of the turn-off angle can't forward the current changing very much. That's the reason, the three energy curves are almost the same and the output torque is also very similar.

Considering the current increasing time as shown in Fig. 7.14, the energy curve is tested with a 3-degree-earlier turn on angle, which is 3 degree before the unaligned position. The energy curve is compared with 0 degree turn-on angle. The curve shows that an advance turn-on angle will provide more electric energy converted to output energy, as shown in Fig. 7.15.

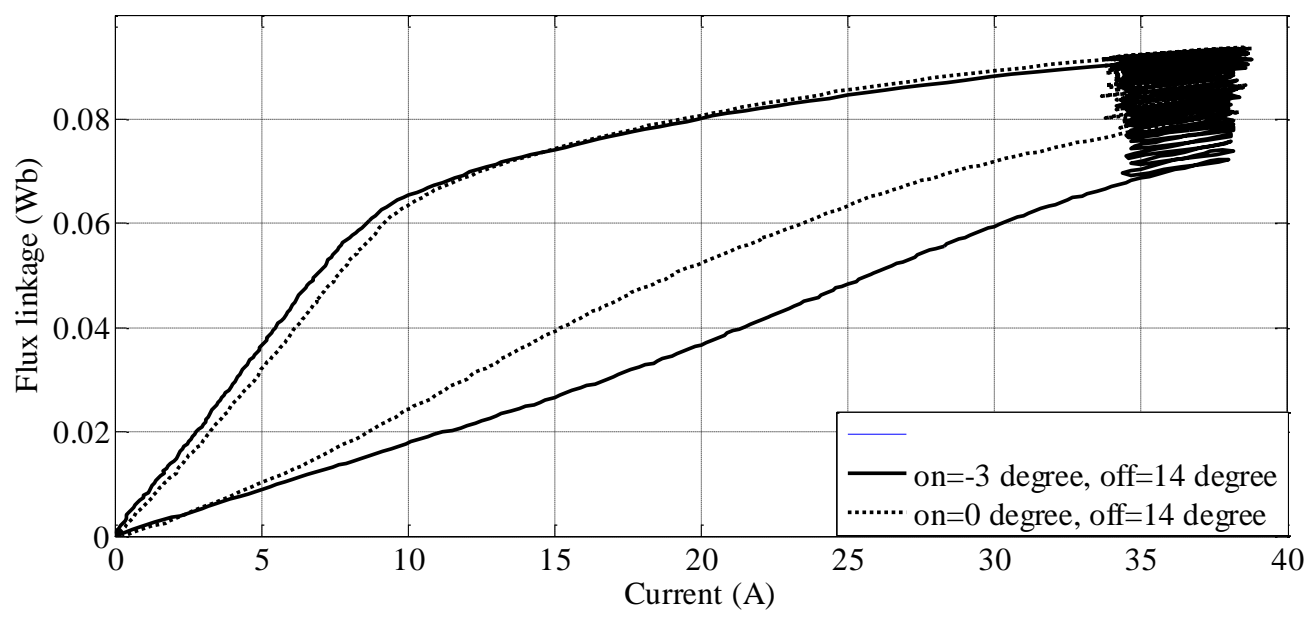

Fig. 7.15. Energy curve comparison for different turn-on but constant turn-off angles.

By calculate the area of the closed loop, the totally output energy is calculated. And the mechanical output power is estimated by multiply the machine's speed and its output torque. As the phase resistance is measured during the testing, which equals about 0.1 ohm, the copper losses is also available. At the same time, mechanical losses are already obtained by no-load operation. By summarizing all those components of the power together, efficiency is calculated as shown in Table 7.3. 
M.A.Sc. Thesis - Jianing Lin; McMaster University - Mechanical Engineering.

Table. 7.3. Performance with turn-on of -3 , and turn-off of 14 degree AP control.

\begin{tabular}{l|l}
\hline Voltage & $48 \mathrm{~V}$ \\
\hline Current & $36 \mathrm{~A}$ \\
\hline RPM & 500 \\
\hline Control & APC \\
\hline Turn-on angle & -3 degree \\
\hline Turn-off angle & 14 degree \\
\hline Copper resistance & $0.05 \mathrm{ohm}$ \\
\hline Total energy & $471 \mathrm{~W}$ \\
\hline Copper loss & $63 \mathrm{~W}$ \\
\hline Mechanical loss & $3.3 \mathrm{~W}$ \\
\hline Mechanical output & $364 \mathrm{~W}$ \\
\hline Iron loss & $41 \mathrm{~W}$ \\
\hline Efficiency & $77.3 \%$ \\
\hline
\end{tabular}

\subsubsection{Speed-power charactoristics}

By checking the maximum output power at different speed, the speed-torque curve for this machine is obtained, as shown in Fig. 7.16.

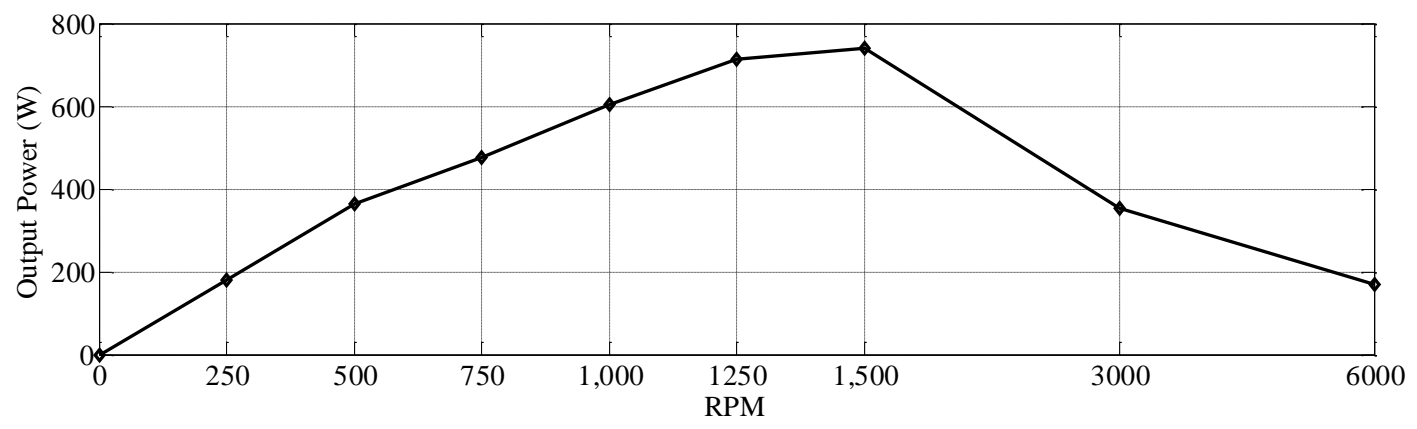

Fig. 7.16. Machine's speed-power curve.

For the speed range of 0 to $1250 \mathrm{rpm}$, the increasing slope is almost constant, which implies a constant torque range. However, which the speed keeps increase, the voltage duty cycle will reach to 1 . So the out power will be almost constant. This is a short range 
M.A.Sc. Thesis - Jianing Lin; McMaster University - Mechanical Engineering.

about 1250 to $1500 \mathrm{rpm}$. However, if the speed still increase, the output power will drop rapidly, as shown from 1500 to 6000rpm.

The flux linkage vs. current curve at speed of 6000rpm is presented in Fig. 7.17. It's clearly seen that, for a high speed, the voltage is not enough to supply the current increasing. So, the current can't reach the rated value. And it will lead to rapid drop of the output power.

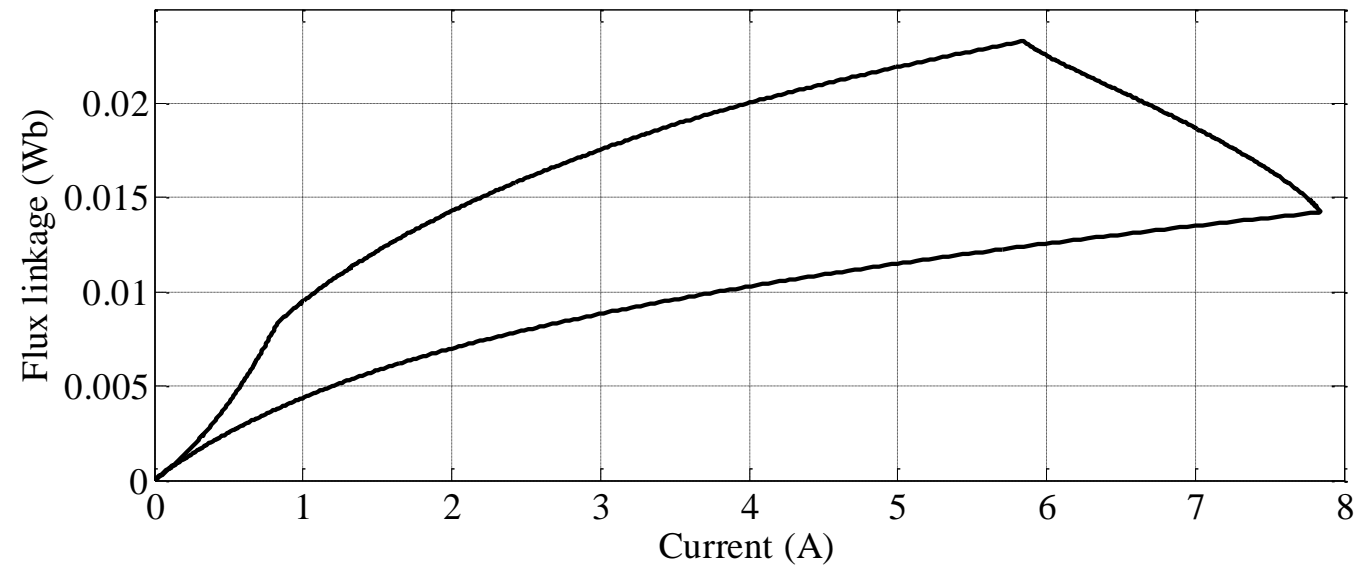

Fig. 7.17. Flux linkage profile at 6000rpm.

\subsection{Conclusions}

The experiment results show that for this short length motor, its end winding influence is much larger than the conventional SRM. Thus, it can't be ignored during the FE analysis.

At the same time, resulting from the manufactory issue, the stator thickness is reduced. This leads to a lower flux-linkage and lower inductance value at aligned position. Together with the end winding influence, which causes a higher inductance value at unaligned position, the output energy curve's area will be definitely reduced. 
M.A.Sc. Thesis - Jianing Lin; McMaster University - Mechanical Engineering.

Thus, the output mechanical power at rated speed can only reach to $364 \mathrm{~W}$, with an efficiency of $77 \%$.

However, the speed-power curve shows that the machine's overload capability is considerable after the rated speed. The constant torque range can even go as far as $1250 \mathrm{rpm}$, whose output power is about $700 \mathrm{~W}$. However, bigger reduction gear ratio is need to for the application of E-bike. 
M.A.Sc. Thesis - Jianing Lin; McMaster University - Mechanical Engineering.

\section{Chapter 8 Conclusions and Future Work}

\subsection{Conclusions}

Considering the special application requirement of E-bike, an external-rotor 500W and 500rpm SRM is designed in this paper. A 3:1 reduction gear box makes its on-road speed legal in Ontario, Canada.

The structure of 6 stator poles and 10 rotor poles makes the motor owns the characteristics of higher torque and lower torque ripple, compared with conventional 6/4 motor. Following the output power equation and analysis of each geometry diameter, the flux-linkage and torque profile of the optimal design solution is studied in 2D FEA. It's verified that the output torque and power can achieve a typical unban E-bike velocity cycle for about 3h, with a chosen 48v 10Ah Samsung LiMn battery. However, as for the concentrated winding, NSNSNS instead of traditional NNNSSS is proved to have a higher and more consistent output torque.

Compared with other kind of electric machine, SRM suffers more serious torque ripples, caused by its simple double salient structure and serious nonlinearity. The torque sharing function control is verified with better torque ripple reduction function, than the traditional turn-on turn-off angle control; however, this is at the expense of high current and losses.

The thermal analysis is also conducted to check the safety of this machine. The statics temperature is within $80^{\circ} \mathrm{C}$ according to the average losses calculated from the velocity cycle. This is much lower than $180^{\circ} \mathrm{C}$ which is unsafe for the motor's insulation thermo- 
M.A.Sc. Thesis - Jianing Lin; McMaster University - Mechanical Engineering.

stabilization requirement. No extra cooling is needed. Prototype machine is manufactured with a hollow static shaft and a rotational rotor case.

There are two parts for the experimental test. The static test presents the flux-linkage and torque profile for different current at different positions. However, the difference between the test results and 2D FEA shows a great necessary to consider the end winding for a small thickness machine. And the decreasing of the stator lamination stack, which is caused by the manufactory error also influence the machine's performance, which leads to a deeper saturation at aligned position. The dynamic test shows that the maximum output mechanical power at $500 \mathrm{rpm}$ is about $364 \mathrm{~W}$, with an efficiency of $77 \%$. The output power is lower than the analysis results, which is mainly caused by the end winding and stator stack thickness reduction. However, it shows that the machine has very good overload ability. The maximum speed can reach to $1250 \mathrm{rpm}$ for the constant torque region.

\subsection{Future Work}

As the experiments propose a difference with the analysis results. Future work on the control strategies based on the experimental data needs to be conducted. At the same time, the thermal analysis is also need to be checked with the future experimental test. In addition, the modeling method for end winding of short thickness machine requires more study in the future.

Also, the whole E-bike system is planned to be set up in the soon future to study the on-road operation of this machine. More optimization both on the structure and control strategies will be conducted in the future for the SRM on E-bike project. 
M.A.Sc. Thesis - Jianing Lin; McMaster University - Mechanical Engineering.

\section{References}

[1] New national standards on e-bikes to be out soon. [Online]. Available: http://www.chinadailyapac.com/article/new-national-standards-e-bikes-be-out-soon, last accessed on July 31, 2013.

[2] On your e-bike: European craze powers into UK. [Online]. Available: http://www.guardian.co.uk/lifeandstyle/2012/oct/12/e-bike-european-craze-uk, last accessed on July 31, 2013.

[3] A. Muetze and Y. C. Tan, "Electric bicycles-a performance evaluation," IEEE Ind. Appl. Mag., vol. 13, no. 4, pp. 12-21, July-Aug. 2007.

[4] Cost of operating electric bike. [Online]. Available: http://www.ecowheelz.com/articles/cost-of-operating-electric-bicycle.php, last accessed on July 31, 2013.

[5] Vehicles and distance travelled in Canada, by type of vehicle Canada. [Online]. Available: http://www.statcan.gc.ca/daily-quotidien/090714/t090714c1-eng.htm, last accessed on Nov. 24, 2013.

[6] Best Gas Mileage by Vehicle Class. [Online]. Available: http://www.cars.com/go/advice/Story.jsp?section=fuel\&story=mpgClass\&subject=fue IList, last accessed on Nov. 24, 2013.

[7] History of eBIKE. [Online]. Available: http://www.raleighebike.co.uk/Historyl, last accessed on Nov. 24, 2013. 
M.A.Sc. Thesis - Jianing Lin; McMaster University - Mechanical Engineering.

[8] What is the perfect battery? [Online]. Available: http://www.buchmann.ca/article4page1.asp, last accessed on Nov. 24, 2013.

[9] C. C. Chan, "The state of the art of electric and hybrid vehicles," in Proc. IEEE, vol. 90, no. 2, pp. 247-275, Feb. 2002.

[10] K. Rajashekara, "History of electric vehicles in General Motors," IEEE Trans. Ind. Appl., vol. 30, no. 4, pp. 897-904, July/Aug. 1994.

[11] K. M. Rahman, B. Fahimi, G. Suresh, A. V. Rajarathnam and M. Ehsani, "Advantages of switched reluctance motor applications to EV and HEV: design and control issues," IEEE Trans. Ind. Appl., vol. 36, no. 1, pp. 111-121, Jan./Feb. 2000.

[12] E. Starschich and A. Muetze, "Comparison of the performances of different geared brushless-DC motor drives for electric bicycles," in Proc. IEEE Electr. Mach. Drives Conf., Antalya, Turkey, 2007, pp. 140-147.

[13] W. Chlebosz, G. Ombach, and J. Junak, "Comparison of permanent magnet brushless motor with outer and inner rotor used in e-bike," in Proc. Electrical Machines, 2010 XIX International Conference on, Rome, Italy, 2010, pp. 1-5.

[14] K. Watanabe, H. Sakata, and H. Ariyoshi, "Linear electric bicycle using simplified inverte," in Proc. 2nd Int. Power Electronics and Motion Control Conf., Hangzhou, China, 1997, pp. 832-837.

[15] R. Raute and N. Ertugrul, "Sensorless permanent magnet AC motor drive with near zero-speed operation for electric-assisted bicycle," in Proc. Power Eletronics and Application, 2005 European Conference on, Dresden, 2005, pp. 5024-5033. 
M.A.Sc. Thesis - Jianing Lin; McMaster University - Mechanical Engineering.

[16] E. Starschich and A. Muetze, "Comparison of the performance of different geared brushless-DC Motor drive for electric bicycle," in Proc. IEEE Electr. Mach. Drives Conf., Antalya, 2007, pp. 140-147.

[17] BionX Motors. [Online]. Available: http://www.bionxinternational.com/bionxinternational-north-america/products/bike-retrofit-systems/motors/, last accessed on Nov. 24, 2013.

[18] M. Zeraoulia, M. EI H. Benbouzid, and D. Diallo, "Electric motor drive selection issue for HEV propulsion system: a comparative Study," IEEE Trans. Veh. Technol., vol. 55, no. 6, pp. 1756-1764, Nov. 2006.

[19] P. Andrada, M. Torrent, B. Blanque, and J. I. Perat, "Switched reluctance drive for electric vehicle application," in Proc. International Conf. on Renewable energies and power quality. Spain, 2003, pp. 373-379.

[20] L. Chang, "Comprison of AC drives for electric vehicles - a report on experts' opinion survey," . IEEE Trans. Aerosp. Electron. Syst., vol. 9, no. 8, pp. 7-11, Aug. 1994.

[21] B. D. Bedford, "Compatible brushless reluctance motors and controlled switch circuits," US. Patents number 3678352, 1972.

[22] P. J. Lawrenson, J. M. Stephenson, P. T. Blenkinsop, J. Corda, and N. N. Fulton, "Variable-speed switched reluctance motors," IEE Electric Power Appl., vol. 127, no. 4, pp. 253-265, July 1980. 
M.A.Sc. Thesis - Jianing Lin; McMaster University - Mechanical Engineering.

[23] S. Tadashi, P. C. Kjaer, C. Cossar, and T. J. E. Miller, "Fault tolerant operation of single phase switched reluctance generator," in Proc. IEEE Appl. Power Electron. Conf., Atlanta, Georgia, 1997, pp. 553-558.

[24] W. Pengov, J. R. Jr., Hendershot, and T. J. E. Miller, "A new low-noise twophase switched reluctance motor," in Proc. IEEE Electr. Mach. Drives Conf., San Antonio, Texas, 2005, pp. 1281-1284.

[25] L. Z. Sun, G. Yang, and Q. Feng, "Study on the rotor levitation of one high speed switched reluctance motor," in Proc. 32th IEEE Annu. Conf. Ind. Electron., Paris, France, 2006, pp. 1322-1325.

[26] L. Takau and S. Round. "Design of a switched reluctance motor for and electric vehicle," in Proc. Australasian University Power Engineering Conf., Christchurch, New Zealand, 2002.

[27] H. Chen and C. Zhang, "A three phase 6/8 structure switched reluctance motor drive," in Proc. IEEE Power System Technology Conf., Perth, Australia, 2000, pp. 195-200.

[28] J. Lin, K. W. Cheng, Z. Zhang, and X. Xue, "Experimental investigation of inwheel switched reluctance motor drive system for future electric vehicle," in Proc. IEEE Power Electron. Syst. Appl., Hong Kong, 2009, pp. 1-6.

[29] H. Chen, "The switched reluctance motor drive for application in electric bicycle," IEEE International Symposium on Ind. Electron., vol. 2, pp. 1152-1156, Jun. 2001.

[30] P. J. Lawrenson, “A brief status review of switched reluctance motor drives," EPE Journal, vol.2, no. 3, pp-133-144, 1992. 
M.A.Sc. Thesis - Jianing Lin; McMaster University - Mechanical Engineering.

[31] M. Jahanmahin, A. Hajihosseinlu, E. Afjei, A. Siadatan, and A. Tavakoli, "A novel multilayer 8 by 4 switch reluctance machine with ripple reduction," Int. Symp. Power Electron. Power Electron. Electr. Drives, pp. 536-540, Jun. 2012.

[32] Y. Takano, M. Takeno, N. Hoshi, A. Chiba, M. Takemoto, S. Ogasawara, and M. A. Rahman, "Design and analysis of a switched reluctance motor for next generation hybrid vehicle without PM materials," in Proc.2010 Int. Power Electron. Conf., Sapporo, 2010, pp. 1801-1806.

[33] A. Chiba, Y. Takano, S. Member, and M. Takeno, “Torque density and efficiency improvements of a switched reluctance motor without rare-earth material for hybrid vehicles," IEEE Transactions on Industry Applications, vol. 47, no. 3, pp. 1240-1246, 2011.

[34] K. Kiyota, S. Member, and A. Chiba, "Design of switched reluctance motor competitive to $60 \mathrm{~kW}$ IPMSM in third generation hybrid electric vehicle," IEEE Transactions on Industry Applications, vol. 48, no. 6, pp. 2303-2309, 2012.

[35] R. Krishnan, Switched Reluctance Motor Drives: Modeling, Simulation, Analysis, Design, and Application,p 0-8493-0838-0, Book, Bca Raton, FL: CRC, 2001.

[36] Different Rules for Electric Bikes and Mopeds/ Scooters. [Online] Available: http://www.icbc.com/registration-licensing/offroad-other/low-powered/motorassisted, last accessed on Nov. 24, 2013.

[37] D. M. Sousa, P. J. C. Branco, and J. A. Dente, "Electric bicycle using batteries and super-capacitors," in Proc. Power Eletronics and Application, 2007 European Conference on, Aalborg, Netherlands, Sep. 2007, pp. 1-10. 
M.A.Sc. Thesis - Jianing Lin; McMaster University - Mechanical Engineering.

[38] E. A. Lomonova, A. J. A. Vandenput, and J. Rubá, "Development of an Improved Electrically Assisted Bicycle," in Proc. 2002 IEEE Industry Applications Conference, Pittsburgh, PA, Oct. 2002, pp. 384-389.

[39] How much does it use? [Online] Available: http://ebikes.ca/faq.shtml, last accessed on Nov. 24, 2013.

[40] Magsoft-Product-SPEED. [Online]. Available: http://magsoftflux.com/products/speed, last accessed on Dec. 10, 2013.

[41] Magsoft-Product-Flux. [Online]. Available: $\underline{\text { http://magsoft- }}$ flux.com/products/flux, last accessed on Dec. 10, 2013.

[42] S. Fujishiro, K. Ishikawa, S. Kikuchi, K. Nakamura, and O. Ichinokura, "Design of outer-rotor-type multipolar SRM for EV," Journal of Applied Physics, vol. 99, no. 8, pp. 08R324 - 08R324-3, Apr. 2006.

[43] T. F. Chan, "In-wheel permanent-magnet brushless DC motor drive for an electric bicycle," IEEE Trans. Energy Convers., vol. 17, no. 2, pp. 229-233, Jun. 2002.

[44] T. J. E. Miller, "Switched reluctance motors and their control," in Monographs in Electrical and Electronic Engineering, vol. 31. Oxford, U.K.: Clarendon, 1993.

[45] B. Bilgin, A. Emadi, and M. Krishnamurthy, "Design considerations for switched reluctance machines with a higher number of rotor poles," IEEE Trans. Ind. Electron., vol. 59, no. 10 , pp. 3745-3756, Oct. 2012.

[46] P. C. Desai, M. Krishnamurthy, N. Schofield, and A. Emadi, "Novel switched reluctance machine configuration with higher number of rotor poles than stator poles: 
M.A.Sc. Thesis - Jianing Lin; McMaster University - Mechanical Engineering.

concept to implementation," IEEE Trans. Ind. Electron., vol. 57, no. 2, pp. 649-659, Feb. 2010.

[47] M. Illic-Spong, T. J. E. Miller, and S. R. MacMinn, "Instantaneous torque control of electric motor drives, ” IEEE Trans. Power Electron., vol. 2, no. 1, pp. 55-61, Jan. 1987.

[48] D. S. Schramm, B. W. Williams, and T. C. Green, "Torque ripple reduction of switched reluctance motors by phase current optimal profiling," in PESC `92 Record. 23rd Annual IEEE Power Electronics Specialists Conference, vol. 2, pp. 857-860, June/July 1992.

[49] N. C. Sahoo, J. X. Xu, and S. K. Panda, "Determination of current waveforms for torque ripple minimization in switched reluctance motors using iterative learning: An investigation," IEE Proc. Electr. Power Appl., vol. 146, no. 4, pp. 369-377, Jul. 1999.

[50] I. Husain, and M. Ehsani, "Torque ripple minimization in switched reluctance motor drives by PWM current control," IEEE Trans. Power Electron. vol. 11, no.1, pp. 83-88, Jan. 1996.

[51] X. D. Xue, K. W. E. Cheng, and S. L. Ho, "Optimization and evaluation of torque-sharing functions for torque ripple minimization in switched reluctance motor drives," IEEE Trans. Ind. Electron., vol. 24, no. 9, pp. 2076-2090, Sept. 2009.

[52] M. Ilic'-Spong, R. Marino, S. Peresada, and D. Taylor, "Feedback linearizing control of a switched reluctance motors," IEEE Trans. Automat. Contr., vol. 32, pp. 371-379, May 1987. 
M.A.Sc. Thesis - Jianing Lin; McMaster University - Mechanical Engineering.

[53] W. Perruquetti, and Jean-Pierre Barbot, "Sliding Mode Control in Engineering," Publication Date: January 29, 2002| ISBN-10: 0824706714| ISBN-13: 9780824706715 | Edition: $1^{\text {st }}$.

[54] S. Mir, M. Elbuluk, and I. Husain, "Torque ripple minimization in switched reluctance motors using adaptive fuzzy control," IEEE Trans. Ind. Applicat., vol. 35, pp. 461-468, Mar./Apr. 1999.

[55] D. S. Reay, T. C. Green, and B.W.Williams, "Neural networks used for torque ripple minimization from a switched reluctance motor," in Proc. EPE'93, Brighton, Sept. 1993, pp. 2-7.

[56] J. G. O’Donovan J, P. J. Roche, R. C. Kavanagh, M. G. Egan, and J. M. D. Murphy, "Neural network based torque ripple minimization in a switched reluctance motor," in Proc. IECON'94 - 20th Annual Conference of IEEE Industrial Electronics, Bologna, Sept. 1994, pp. 1226-1231.

[57] Y. Chen, C. L. Chen, Q. Dong, and R. W. Stephenson, "Thermal management for motor," in Proc. 2002. Eighth Intersociety Conference on Thermal and Thermomechanical Phenomena in Electronic Systems, 2002, pp. 545-551.

[58] Y. Huai, R. V. N. Melnik, and P. B. Thogersen, "Computational Analysis of Temperature Rise Phenomena in Electric Induction Motors," Appl. Therm. Eng., vol. 23, no. 7, pp. 779-795, May 2003.

[59] K. Narita, Y. Sakashita, T. Yamada, and K. Akatsu, "Iron loss calculation of PM motor by coupling analysis between magnetic field simulator and control simulator," in Proc. 2009 Int. Conf. Electr. Mach. Syst., Tokyo, Japan, Nov. 2009, pp. 1-6. 
M.A.Sc. Thesis - Jianing Lin; McMaster University - Mechanical Engineering.

[60] D. Staton, A. Boglietti, and A. Cavagnino, "Solving the More Difficult Aspects of Electric Motor Thermal Analysis”, IEEE Trans. Energy Conversion, vol. 20, no. 3, pp. 620-628, Sept. 2005.

[61] JMAG Application Note, Thermal Analysis of an IPM Motor. [Online]. Available: http://www.jmag-international.com/catalog/18_IPMMotor_Thermal.html, last accessed on Nov. 24, 2013.

[62] Wire gauge and current limits including skin depth and strength. [Online]. Available: http://www.powerstream.com/Wire_Size.htm, last accessed on Dec. 18, 2013.

[63] Electrical steel sheet. [Online]. Available: $\underline{\text { http://www.jfe- }}$ steel.co.jp/en/products/electrical/, last accessed on Dec. 18, 2013.

[64] Aluminum.

$$
\text { [Online]. }
$$

Available: http://www.mit.edu/ 6.777/matprops/aluminum.htm, last accessed on Dec. 18, 2013. 
M.A.Sc. Thesis - Jianing Lin; McMaster University - Mechanical Engineering.

\section{Appendix}

The appendix shows drawings for some important prototype machine parts, which include stator lamination, rotor lamination, shaft, left case end, left case, right case, left bracket, right bracket, bracket triangle, bracket bottom and load cell arm, as all shown in the following figures. All the dimensions are in millimeter and there is no scale for the drawings.

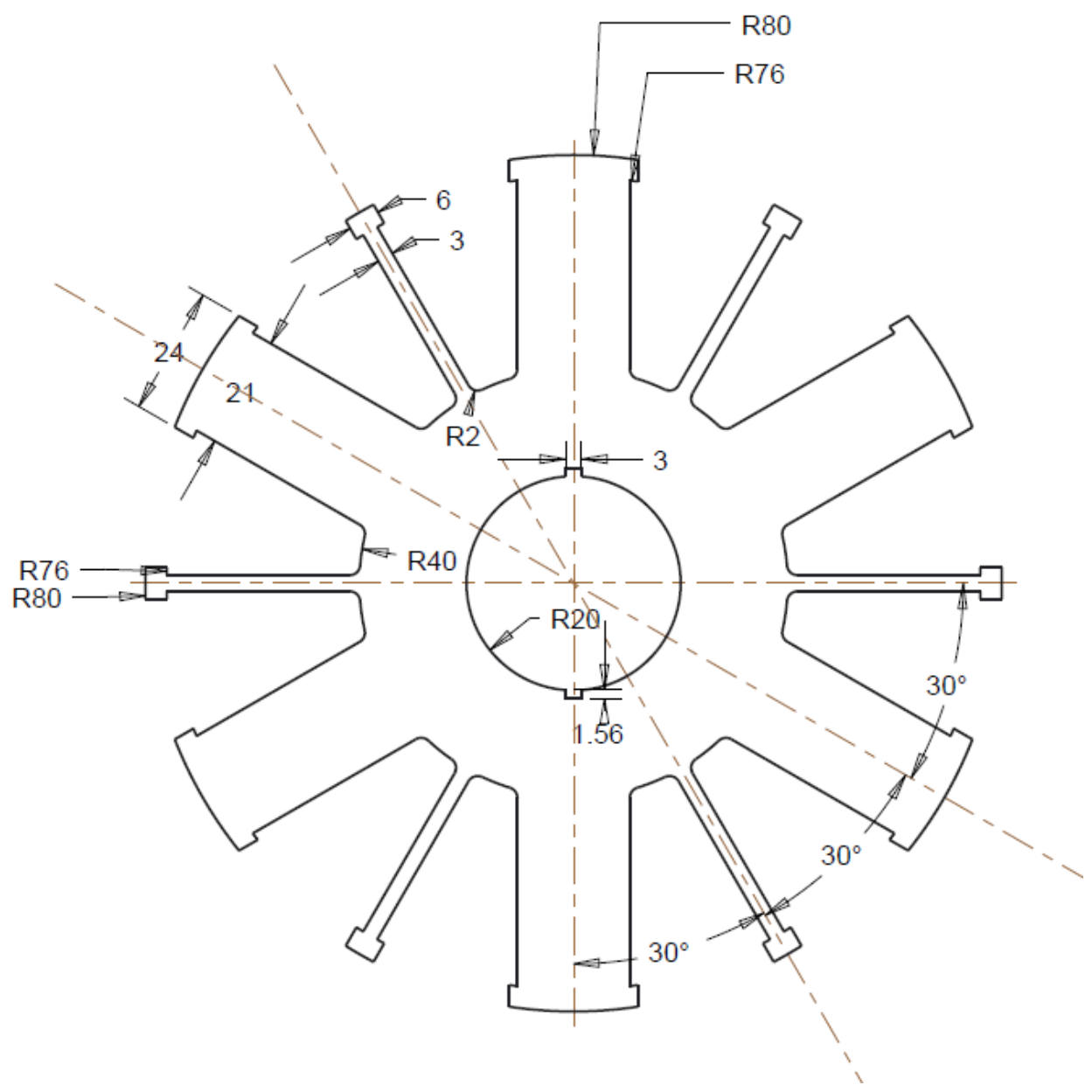

Drawing 1. Stator lamination. 
M.A.Sc. Thesis - Jianing Lin; McMaster University - Mechanical Engineering.

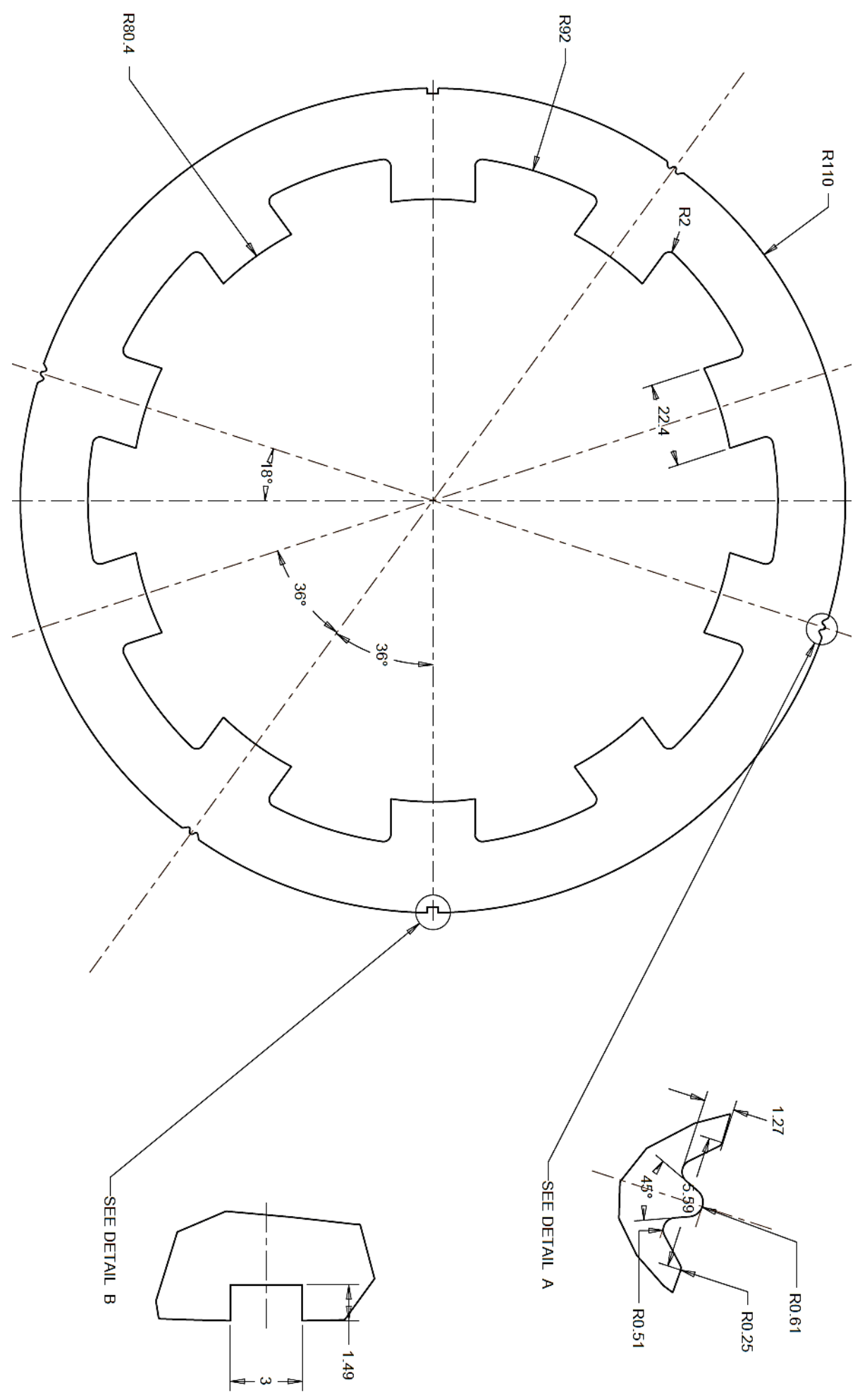

Drawing 2. Rotor lamination. 
M.A.Sc. Thesis - Jianing Lin; McMaster University - Mechanical Engineering.
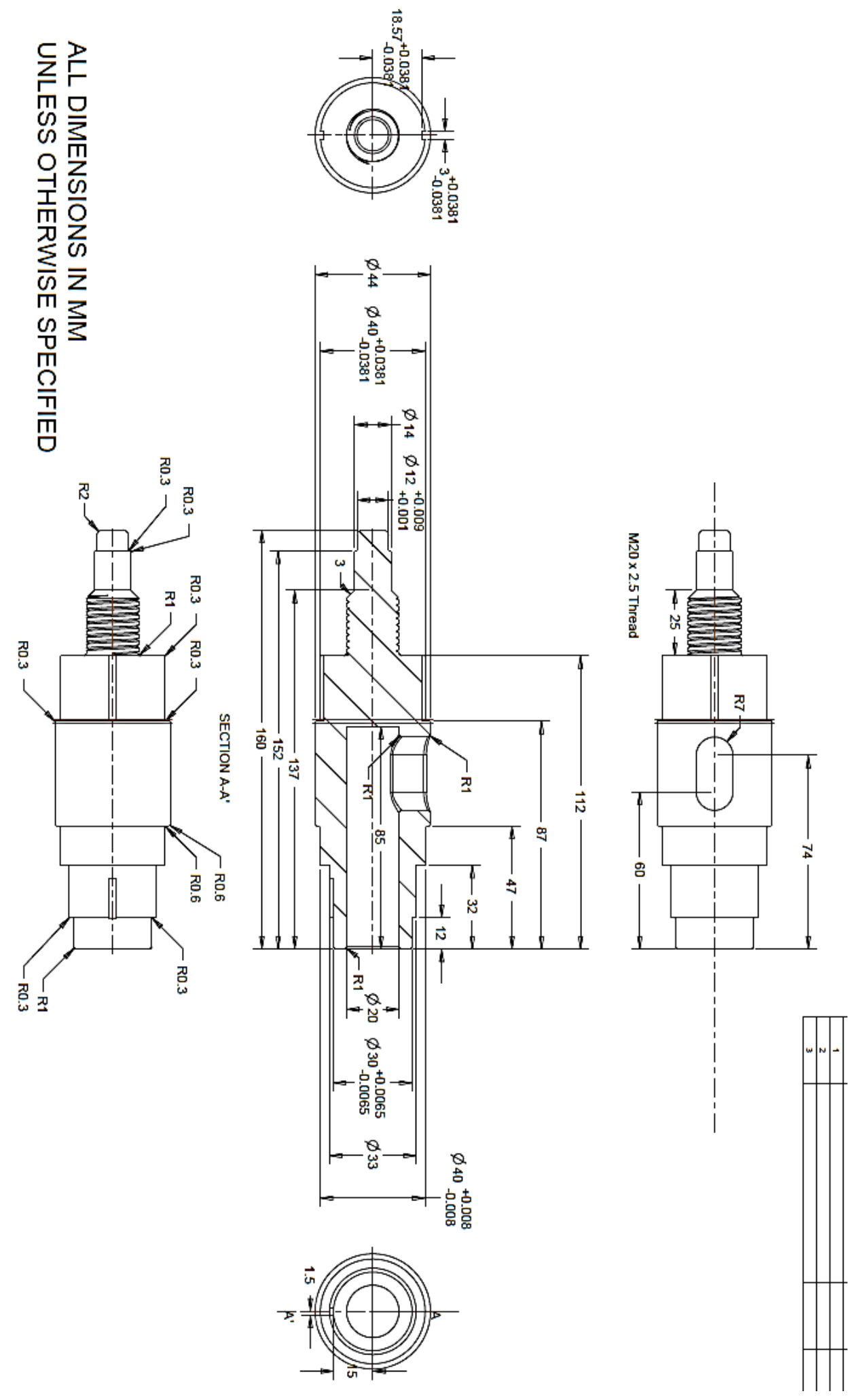

Drawing 3. Shaft. 
M.A.Sc. Thesis - Jianing Lin; McMaster University - Mechanical Engineering.

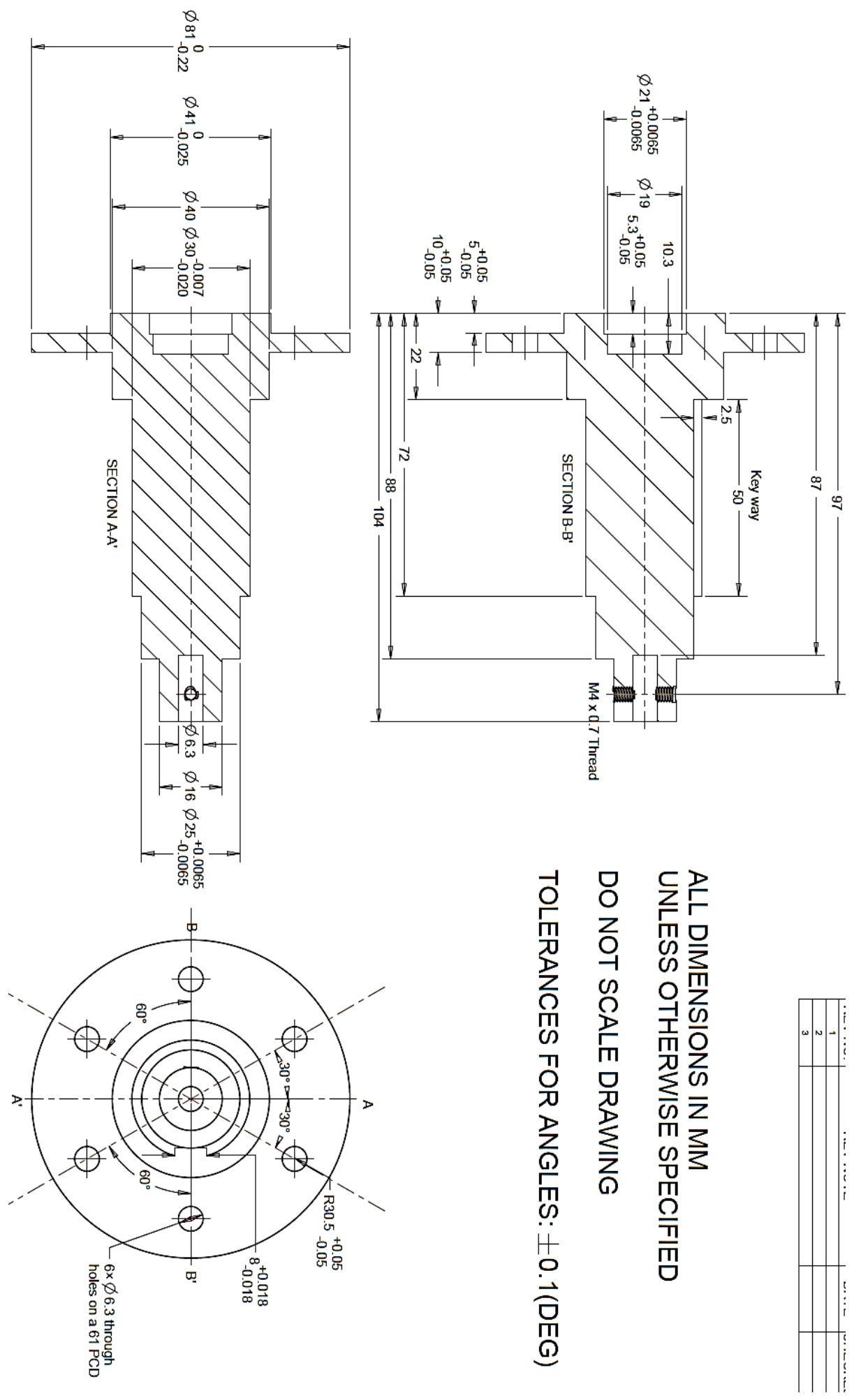

Drawing 4. Left case end. 
M.A.Sc. Thesis - Jianing Lin; McMaster University - Mechanical Engineering.
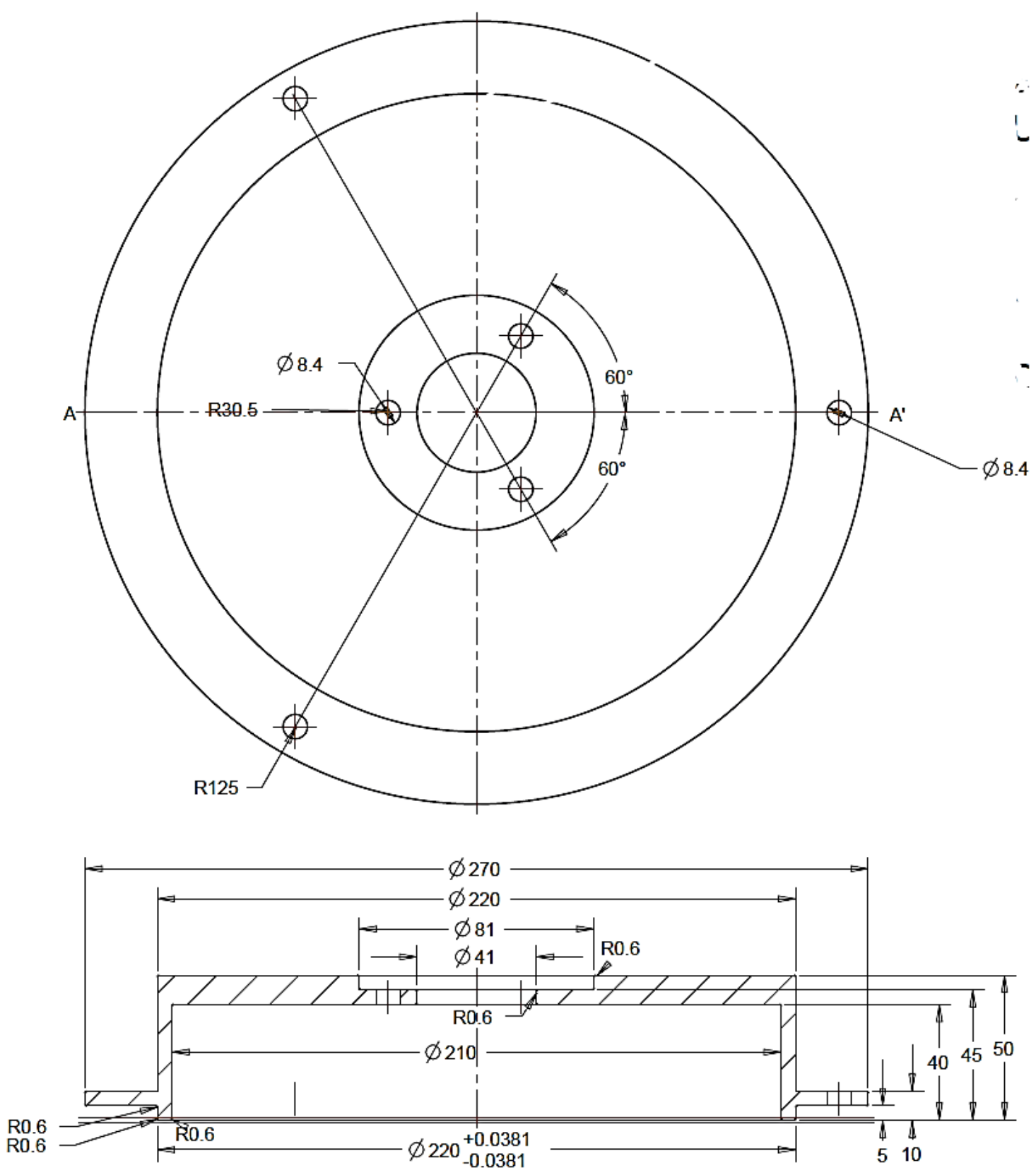

Drawing 5. Left case. 
M.A.Sc. Thesis - Jianing Lin; McMaster University - Mechanical Engineering.
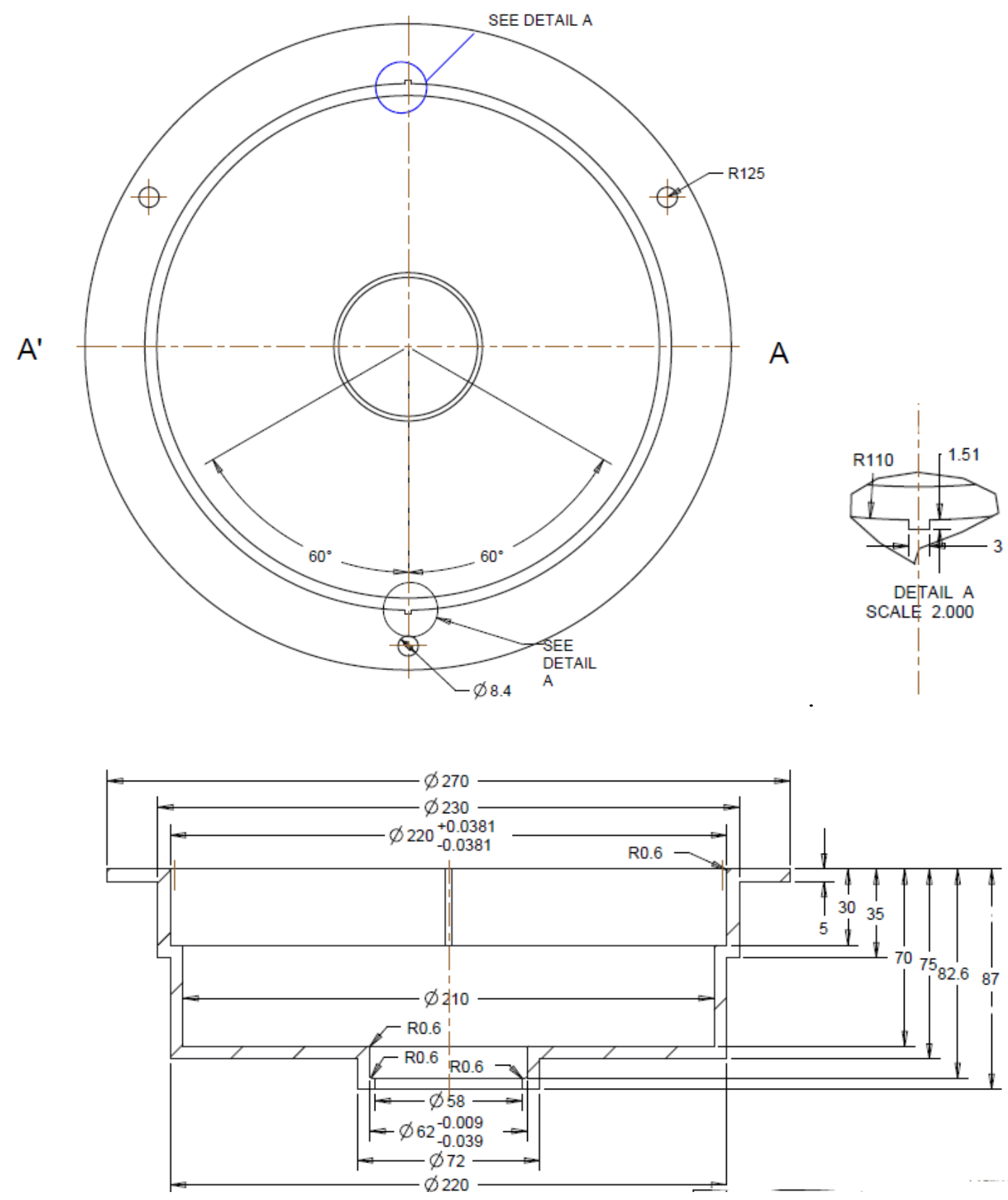

Drawing 6. Right case. 
M.A.Sc. Thesis - Jianing Lin; McMaster University - Mechanical Engineering.

$$
\begin{aligned}
& \stackrel{0}{\mathcal{N}} \\
& \stackrel{M}{\omega} \\
& \stackrel{\omega}{\Phi}
\end{aligned}
$$
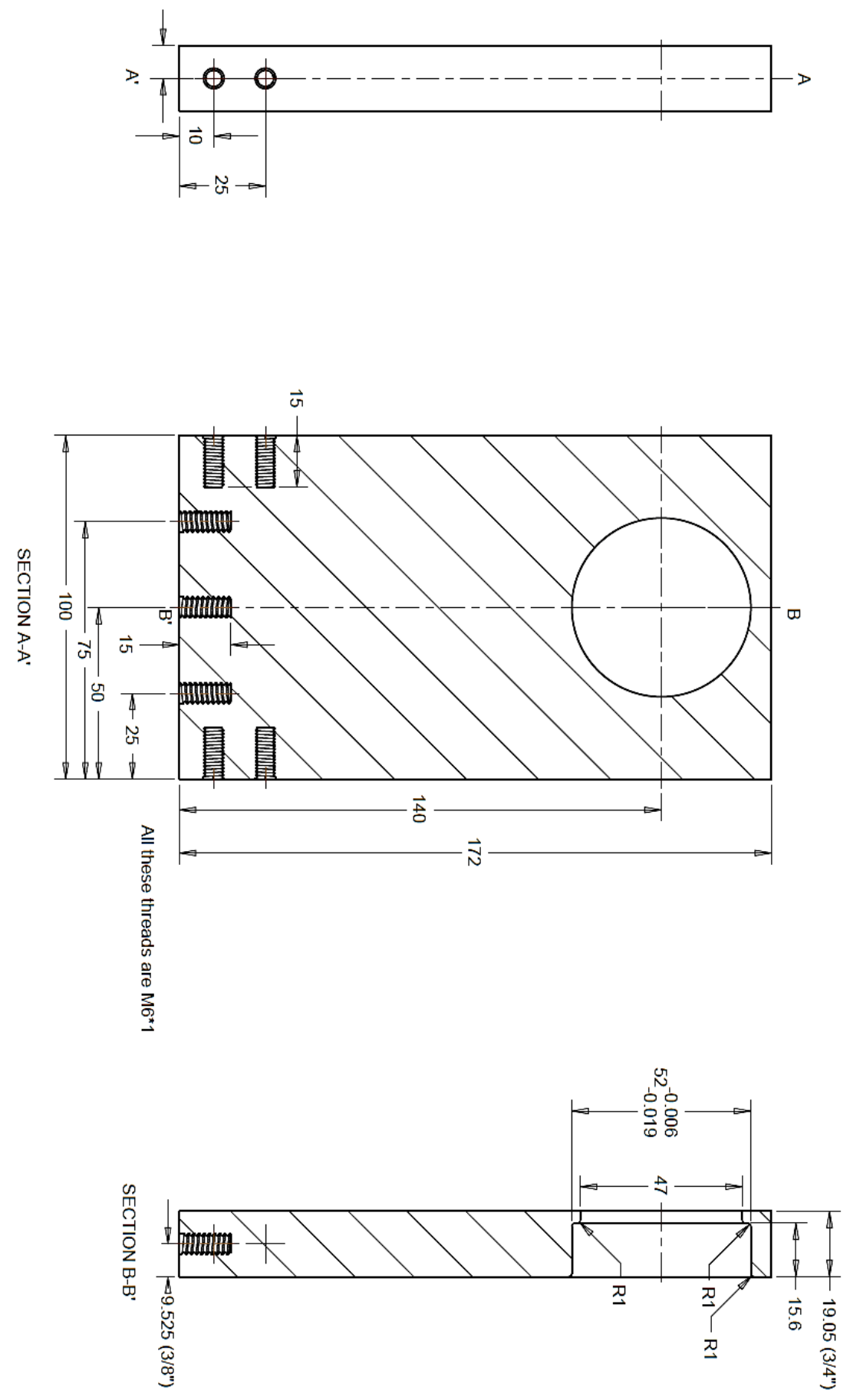

Drawing 7. Left Bracket. 
M.A.Sc. Thesis - Jianing Lin; McMaster University - Mechanical Engineering.
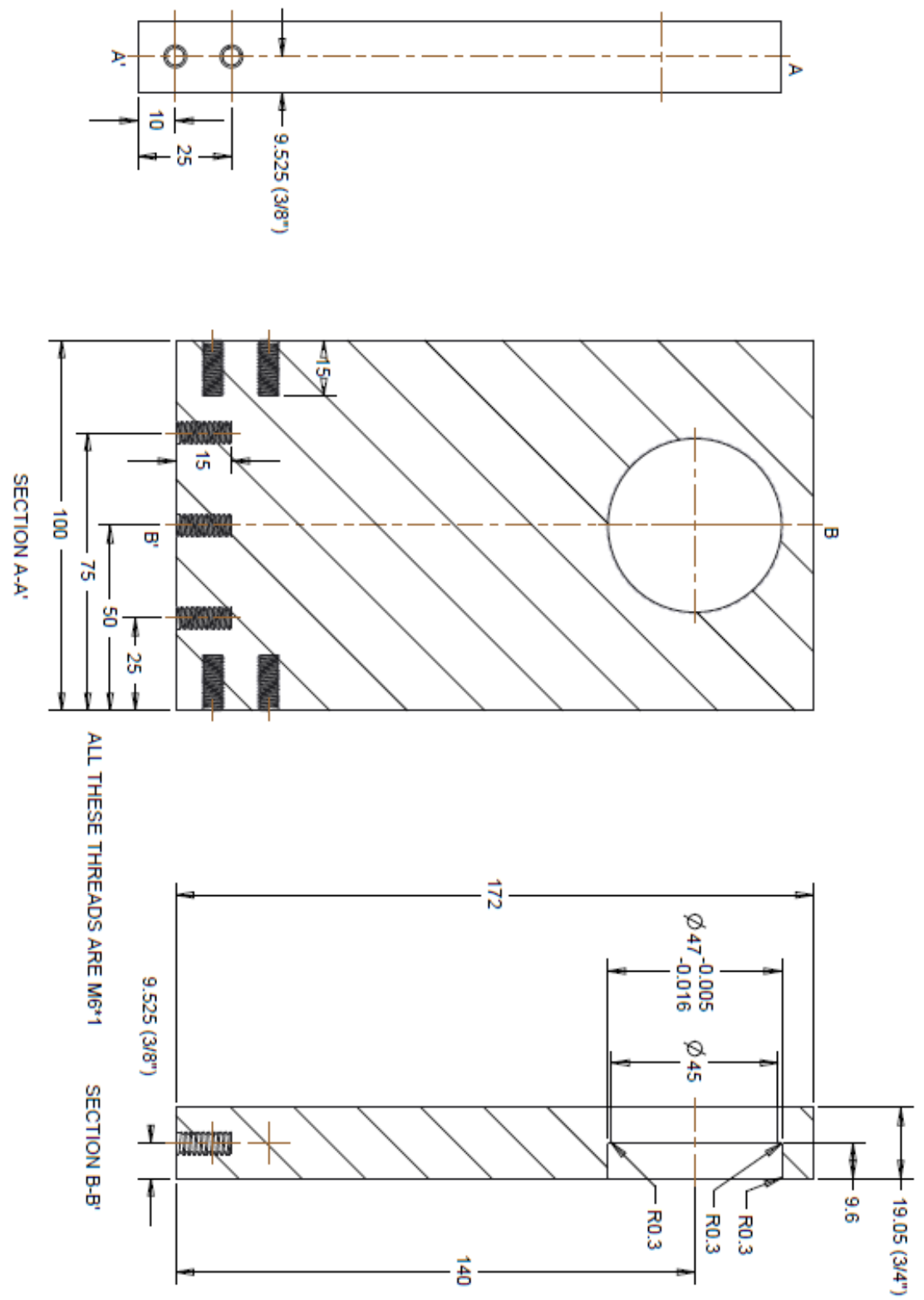

Drawing 8. Right bracket. 
M.A.Sc. Thesis - Jianing Lin; McMaster University - Mechanical Engineering.
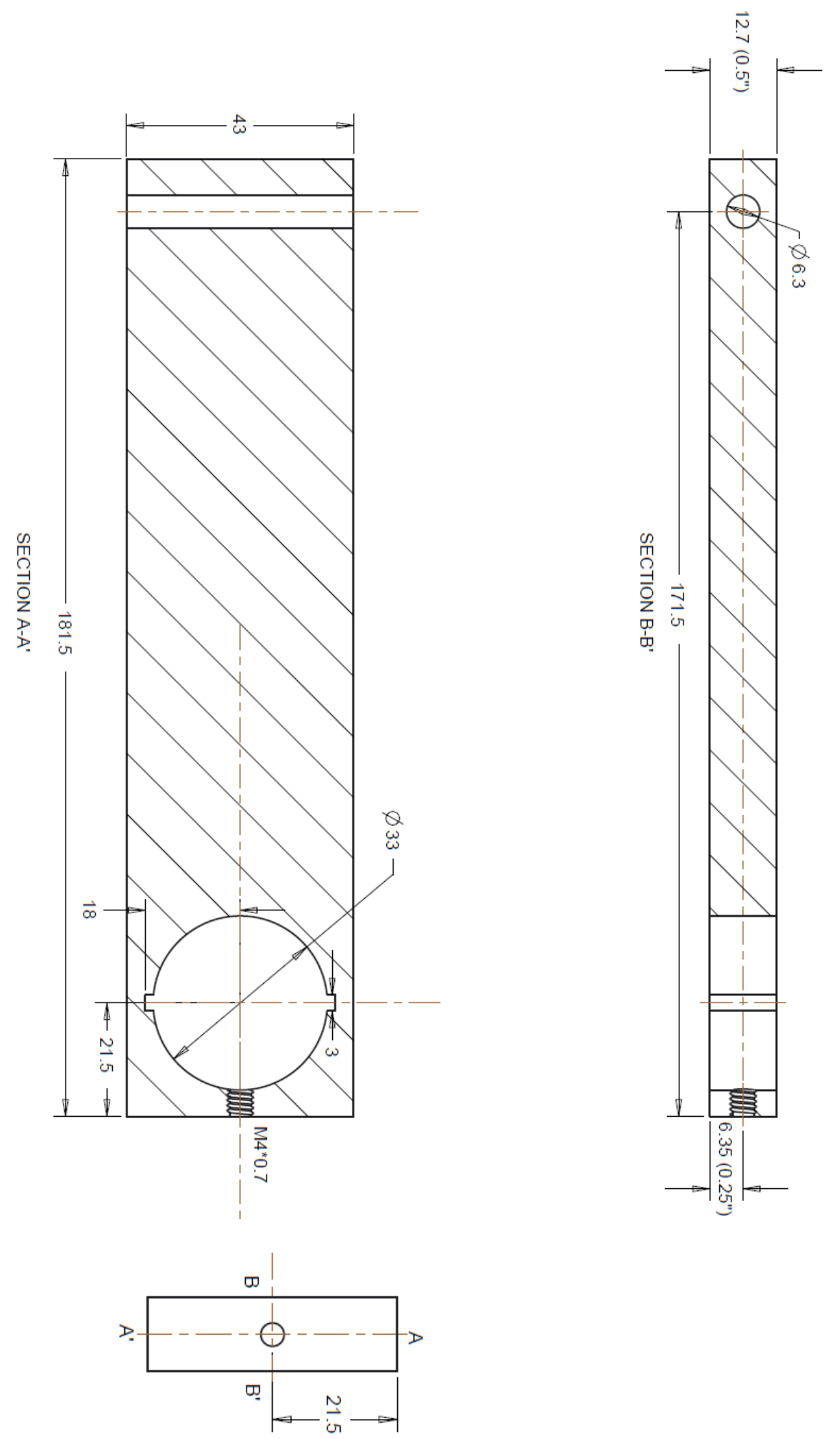

Drawing 9. Load cell arm. 
M.A.Sc. Thesis - Jianing Lin; McMaster University - Mechanical Engineering.

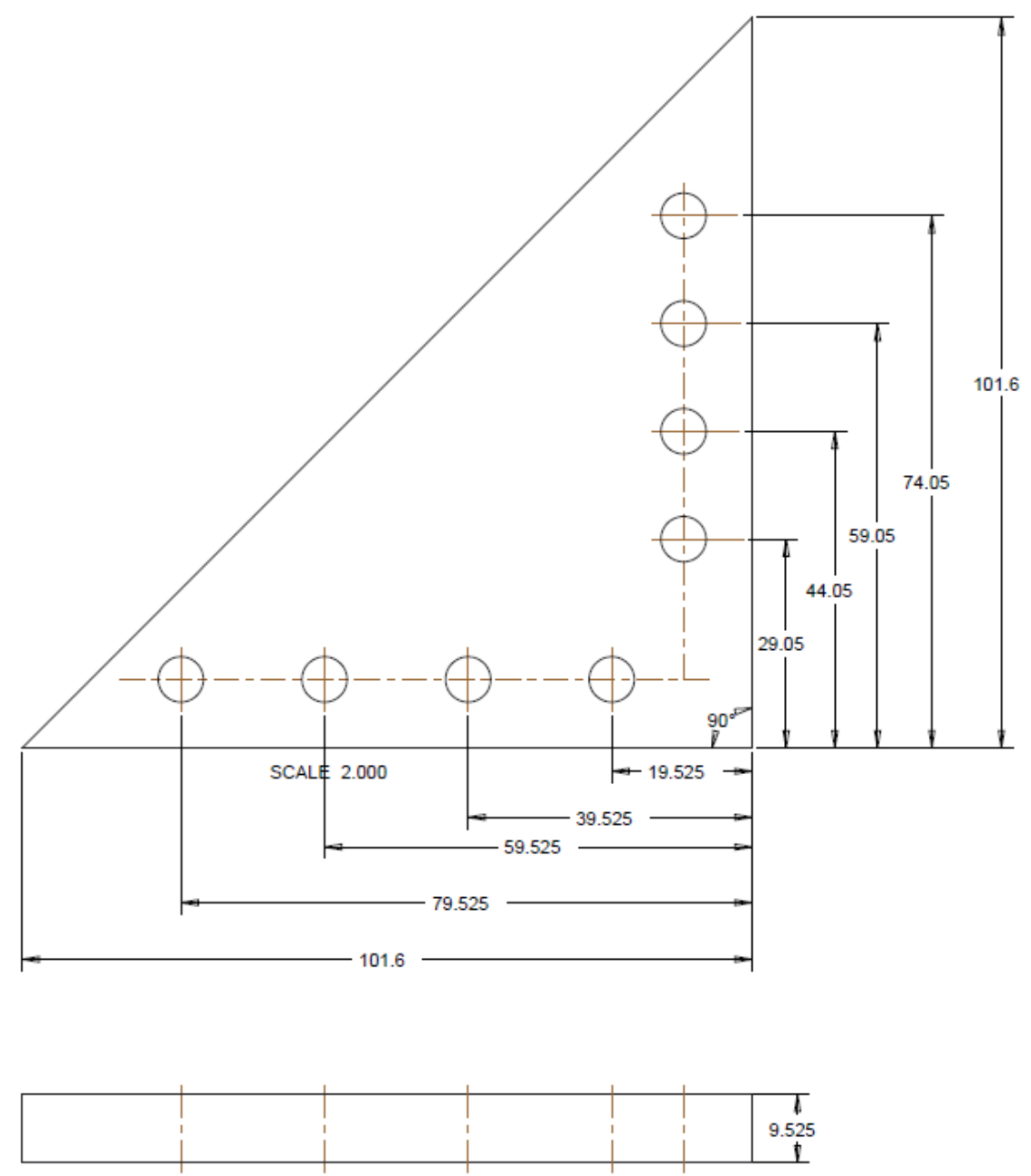

Drawing 10. Bracket triangle. 
M.A.Sc. Thesis - Jianing Lin; McMaster University - Mechanical Engineering.
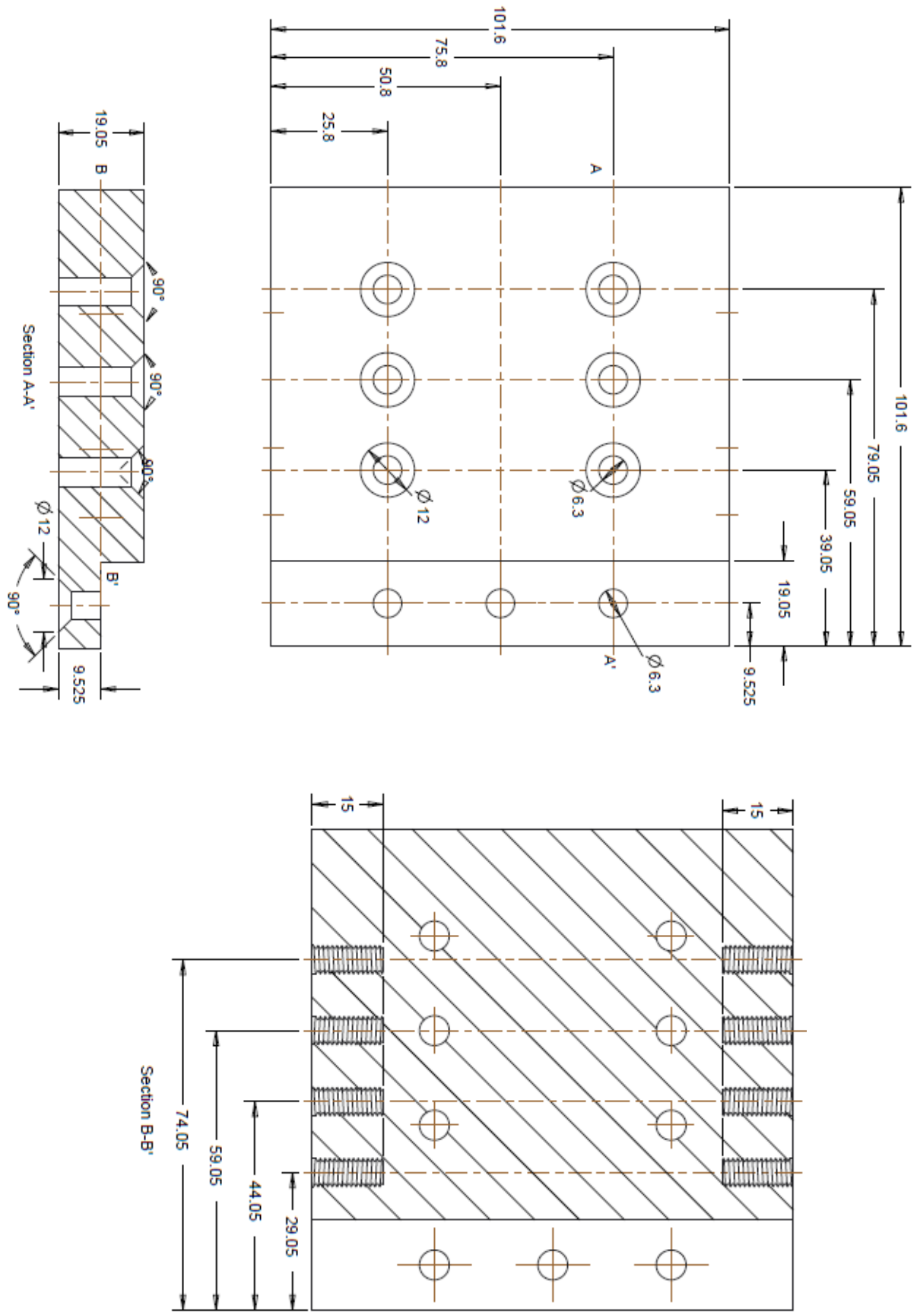

Drawing 11. Bracket bottom. 